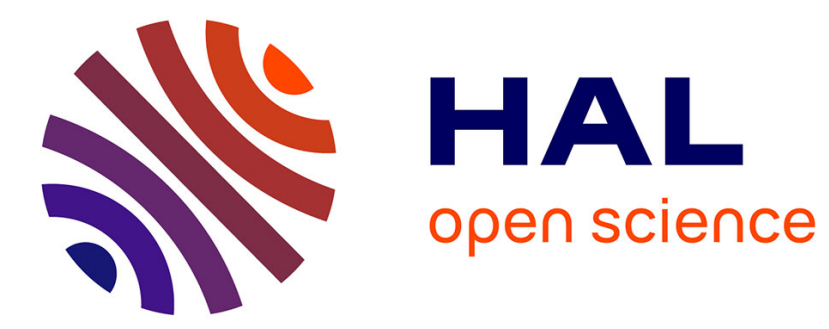

\title{
A FULL DISCRETISATION OF THE TIME-DEPENDENT BOUSSINESQ (BUOYANCY) MODEL WITH NONLINEAR VISCOSITY
}

Rim El Dbaissy $\dagger \ddagger$, Frédéric Hecht, Gihane Mansour, Toni Sayah

\section{- To cite this version:}

Rim El Dbaissy $\dagger \ddagger$, Frédéric Hecht, Gihane Mansour, Toni Sayah. A FULL DISCRETISATION OF THE TIME-DEPENDENT BOUSSINESQ (BUOYANCY) MODEL WITH NONLINEAR VISCOSITY. 2017. halshs-01646611v2

\section{HAL Id: halshs-01646611 \\ https://shs.hal.science/halshs-01646611v2}

Preprint submitted on 4 Dec 2017

HAL is a multi-disciplinary open access archive for the deposit and dissemination of scientific research documents, whether they are published or not. The documents may come from teaching and research institutions in France or abroad, or from public or private research centers.
L'archive ouverte pluridisciplinaire HAL, est destinée au dépôt et à la diffusion de documents scientifiques de niveau recherche, publiés ou non, émanant des établissements d'enseignement et de recherche français ou étrangers, des laboratoires publics ou privés. 


\title{
A FULL DISCRETISATION OF THE TIME-DEPENDENT BOUSSINESQ (BUOYANCY) MODEL WITH NONLINEAR VISCOSITY
}

\author{
RIM ALDBAISSY ${ }^{\ddagger}$, FRÉDÉRIC HECHT $^{\dagger}$, GIHANE MANSOUR $^{\ddagger}$, AND TONI SAYAH $^{\ddagger}$
}

\begin{abstract}
In this article, we study the time dependent Boussinesq (buoyancy) model with nonlinear viscosity depending on the temperature. We propose and analyze first and second order numerical schemes based on finite element methods. An optimal a priori error estimate is then derived for each numerical scheme. Numerical experiments are presented that confirm the theoretical accuracy of the discretization.

Keywords. Boussinesq, Buoyancy, Navier-Stokes equations; heat equation; finite element method; $a$ priori error estimates.
\end{abstract}

\section{INTRODUCTION.}

Let $\Omega$ be a connected bounded open set in $\mathbb{R}^{d}, d=2$ or 3 , with a lipschitz-continuous boundary $\partial \Omega$, and let $[0, \hat{T}]$ be an interval of $\mathbb{R}$. In this work, we study the Boussinesq approximation (buoyancy) where the viscosity of the fluid $\nu$ depends on the temperature. This system can be represented by the Navier-Stokes equations of an incompressible fluid coupled with the heat equation, where the external body force $\mathbf{f}$ and the viscosity $\nu$ depend on the temperature:

$$
\begin{aligned}
\frac{\partial \mathbf{u}}{\partial t}(\mathbf{x}, t)+(\mathbf{u}(\mathbf{x}, t) . \nabla) \mathbf{u}(\mathbf{x}, t)-\nu(T(\mathbf{x}, t)) \Delta \mathbf{u}(\mathbf{x}, t)+\nabla p(\mathbf{x}, t) & =\mathbf{f}(\mathbf{x}, t, T(\mathbf{x}, t)) & & \text { in } \Omega \times[0, \hat{T}], \\
\frac{\partial T}{\partial t}(\mathbf{x}, t)-\alpha \Delta T(\mathbf{x}, t)+(\mathbf{u}(\mathbf{x}, t) . \nabla) T(\mathbf{x}, t) & =g(\mathbf{x}, t) & & \text { in } \Omega \times[0, \hat{T}], \\
\operatorname{div} \mathbf{u}(\mathbf{x}, t) & =0 & & \text { in } \Omega \times[0, \hat{T}], \\
\mathbf{u}(\mathbf{x}, t) & =0 & & \text { on } \partial \Omega \times[0, \hat{T}], \\
T(\mathbf{x}, t) & =0 & & \text { on } \partial \Omega \times[0, \hat{T}], \\
\mathbf{u}(\mathbf{x}, 0) & =\mathbf{u}_{0} & & \text { on } \Omega, \\
T(\mathbf{x}, 0) & =T_{0} & & \text { on } \Omega,
\end{aligned}
$$

here $\alpha$ is the thermal diffusivity and $g$ is a given data function. The unknowns are the velocity $\mathbf{u}$, the temperature $T$ and the pressure $p$ of the fluid. The function $\nu$ is positive and bounded, while the coefficient $\alpha$ is a positive constant. For the simplicity, the nonlinear system of partial differential equations in (1.1) is provided by the homogeneous Dirichlet conditions.

Many works have been carried out this system. A similar model of (1.1) but for a given external body force $\mathbf{f}$ (independent of $T$ ) has been studied in [2], by using a spectral discretization. As well, in [3,6], the authors study the steady state coupled system of Navier-Stokes and heat equations.

Problem (1.1) is totaly discretized in time and space. We introduce two types of discrete schemes: first order and second order. For both, we establish a priori error estimates in two dimensions based on the discrete Gronawll Lemma. In order to confirm the optimality of the discretization and justify the choice

December 4, 2017.

† Sorbonne Universités, UPMC Univ Paris 06, CNRS UMR 7598, Laboratoire Jacques-Louis Lions, F-75005, Paris, France. E-mails: frederic.hecht@upmc.fr.

¥ Laboratoire de Mathétiques et Applications, Unité de recherche Mathématiques et Modélisation, Faculté des Sciences, Université Saint-Joseph, B.P 11-514 Riad El Solh, Beyrouth 1107 2050, Liban.

E-mails: rim.dbaissy@net.usj.edu.lb, gihane.mansour@usj.edu.lb, toni.sayah@usj.edu.lb. 
of this formulation, several numerical experiments where provided.

The outline of the paper is as follows:

- In Section 2, we introduce some notations and functional spaces useful for the studies of the problem.

- In section 3, we recall and study the variational formulation, the existence and the uniqueness (for $d=2$ ) of the problem.

- Section 4 is devoted to introduce and study the first order descretization, and perform the $a$ priori corresponding error analysis for $d=2$.

- We introduce a second order discrete scheme in Section 5. We also perform and study the a priori error analysis for $d=2$.

- Some numerical experiments are presented in Section 6.

\section{Preliminaries}

In this section, we recall the main notion and results which we use later on. We introduce the Sobolev space

$$
W^{m, r}(\Omega)^{d}=\left\{\mathbf{v} \in L^{r}(\Omega)^{d} ; \partial^{k} \mathbf{v} \in L^{r}(\Omega)^{d}, \forall|k| \leq m\right\},
$$

where $k=\left\{k_{1}, \ldots, k_{d}\right\}$ is a vector of non negative integers, such that $|k|=k_{1}+\ldots+k_{d}$ and

$$
\partial^{k} \mathbf{v}=\frac{\partial^{k} \mathbf{v}}{\partial^{k_{1}} x_{1} \ldots \partial^{k_{d}} x_{d}} .
$$

This space is equipped with the semi-norm

$$
|\mathbf{v}|_{W^{m, r}(\Omega)^{d}}=\left(\sum_{|k|=m} \int_{\Omega}\left|\partial^{k} \mathbf{v}\right|^{r} d \mathbf{x}\right)^{\frac{1}{r}},
$$

and is Banach for the norm

$$
\|\mathbf{v}\|_{W^{m, r}(\Omega)^{d}}=\left(\sum_{l=0}^{m} \int_{\Omega}|\mathbf{v}|_{W^{l, r}(\Omega)^{d}}^{r} d \mathbf{x}\right)^{\frac{1}{r}} .
$$

When $r=2$, this space is the Hilbert space $H^{m}(\Omega)^{d}$. In particular, we consider the following spaces

$$
\begin{gathered}
X=H_{0}^{1}(\Omega)^{d}=\left\{\mathbf{v} \in H^{1}(\Omega)^{d} ; \mathbf{v}_{\mid \partial \Omega}=0\right\}, \\
Y=H_{0}^{1}(\Omega)
\end{gathered}
$$

and its dual $H^{-1}(\Omega)^{d}$.

We shall also introduce

$$
M=L_{0}^{2}(\Omega)=\left\{q \in L^{2}(\Omega) ; \int_{\Omega} q(\mathbf{x}) d \mathbf{x}=0\right\} .
$$

We define the following scalar product in $L^{2}(\Omega)$ :

$$
(v, w)=\int_{\Omega} v(\mathbf{x}) w(\mathbf{x}) d \mathbf{x}, \quad \forall v, w \in L^{2}(\Omega) .
$$

For the functions that vanish on the boundary, we should recall the Sobolev inequalities:

Lemma 2.1. For any $p \geq 1$ when $d=1$ or 2 , or $1 \leq p \leq \frac{2 d}{d-2}$ when $d \geq 3$, there exists two positive constants $S_{p}$ and $S_{p}^{0}$ such that

and

$$
\forall \boldsymbol{v} \in H_{0}^{1}(\Omega)^{d}, \quad\|\boldsymbol{v}\|_{L^{p}(\Omega)^{d}} \leq S_{p}^{0}|\boldsymbol{v}|_{H_{0}^{1}(\Omega)^{d}}
$$

$$
\forall \boldsymbol{v} \in H^{1}(\Omega)^{d},\|\boldsymbol{v}\|_{L^{p}(\Omega)^{d}} \leq S_{p}\|\boldsymbol{v}\|_{H_{0}^{1}(\Omega)^{d}} .
$$

Lemma 2.2. For $d=2$, we have the relation

$$
\|\mathbf{v}\|_{L^{4}(\Omega)^{2}}^{2} \leq 2^{1 / 2}\|\mathbf{v}\|_{L^{2}(\Omega)^{2}}|\mathbf{v}|_{H_{0}^{1}(\Omega)^{2}}
$$


As usual, for handling time-dependent problems, it is convenient to consider functions defined on a time interval $] a, b\left[\right.$ with values in a separable functional space $W$ equipped with a norm $\|\cdot\|_{W}$. Then for any $r \geq 1$, we introduce the space

$$
L^{r}(a, b ; W)=\{f \text { mesurable sur }] a, b\left[; \int_{a}^{b}\|f(t)\|_{W}^{r} d t<\infty\right\} ;
$$

equipped with the norm

$$
\|f\|_{L^{r}(a, b ; W)}=\left(\int_{a}^{b}\|f(t)\|_{W}^{r} d t\right)^{\frac{1}{r}} .
$$

If $r=\infty$, then

$$
L^{\infty}(a, b ; W)=\{f \text { mesurable sur }] a, b\left[; \sup _{t \in[a, b]}\|f(t)\|_{W}<\infty\right\} .
$$

Remark 2.3. $L^{r}(0, \hat{T} ; W)$ is a Banach space if $W$ is a Banach space.

In addition, we define $C^{j}(0, \hat{T} ; W)$ as the space of functions $C^{j}$ in time with values in $W$. Henceforth, we suppose the following hypothesis:

Assumption 2.4. We assume that the data $\mathbf{f}, g$ et $\nu$ verify:

(1) $\mathbf{f}$ can be written as follows:

$$
\mathbf{f}(\mathbf{x}, t, T)=\mathbf{f}_{0}(\mathbf{x}, t)+\mathbf{f}_{1}(\mathbf{x}, T),
$$

where $\mathbf{f}_{0} \in L^{2}\left(0, \hat{T} ; L^{2}(\Omega)^{d}\right)$ and $\mathbf{f}_{1}(T) \in L^{2}(\Omega)^{d}$ is $c_{\mathbf{f}_{1}}^{*}$-lipschitz from $\mathbb{R}$ with values in $\mathbb{R}^{d}$. In addition, we suppose that

$$
\forall \mathbf{x} \in \Omega, \forall \xi \in \mathbb{R},\left|\mathbf{f}_{1}(\mathbf{x}, \xi)\right| \leq c_{\mathbf{f}_{1}}|\xi|,
$$

where $c_{\mathbf{f}_{1}}$ is a positive constant.

(2) $g \in L^{2}\left(0, \hat{T}, L^{2}(\Omega)\right)$,

(3) $\nu \in L^{\infty}(\Omega)$ is continuous. Further more, there exists two positive constants $\hat{\nu}_{1}$ and $\hat{\nu}_{2}$ such that, for any $\theta \in \mathbb{R}$

$$
\hat{\nu}_{1} \leq \nu(\theta) \leq \hat{\nu}_{2} .
$$

Remark 2.5. In the case where $\mathbf{f}_{1}$ denpends on $t, x$ and $T$, we obtain the same results by also setting the following condition:

$\forall x \in \Omega, \forall t \in[0, \hat{T}], \forall \xi \in \mathbb{R}$, we have

$$
\left|\frac{\partial \mathbf{f}_{1}}{\partial t}(x, t, \xi)\right| \leq c_{\mathbf{f}_{1}}^{\prime}|\xi|
$$

where $c_{\mathbf{f}_{1}}^{\prime}$ is a positive constant.

It is similar to write the linear approximation of the function $\mathbf{f}$.

For sake of simplicity, from now on we consider the Assumption (2.4).

Remark 2.6. Henceforth, we use the following relations:

(1) Let $a$ and $b$ be two real numbers.

(a) For any positive real number $\varepsilon$, we have

$$
a b \leq \frac{1}{2 \varepsilon} a^{2}+\frac{1}{2} \varepsilon b^{2} .
$$

(b) We also have

$$
(a-b, a)=\frac{1}{2} a^{2}-\frac{1}{2} b^{2}+\frac{1}{2}(a-b)^{2} .
$$


(2) For any real numbers $a^{n}, a^{n-1}$ and $a^{n-2}$, we have

$$
\begin{aligned}
& 2\left(3 a^{n}-4 a^{n-1}+a^{n-2}, a^{n}\right)=\left|a^{n}\right|^{2}+\left|2 a^{n}-a^{n-1}\right|^{2}+\left|\delta^{2} a^{n-1}\right|^{2}-\left|a^{n-1}\right|^{2}-\left|2 a^{n-1}-a^{n-2}\right|^{2}, \\
& \text { where } \delta^{2} a^{n-1}=a^{n}-2 a^{n-1}+a^{n-2} .
\end{aligned}
$$

\section{Analysis of the COntinuous problem}

We first write down a variational formulation of problem (1.1). Next, we summarize the existence (for $d=2,3$ ) and prove the conditional uniqueness (for $d=2$ ) of the solution.

In order to introduce the variational formulation, we define the two following forms:

$$
c_{\mathbf{u}}(\mathbf{u}, \mathbf{v}, \mathbf{w})=((\mathbf{u} \nabla) . \mathbf{v}, \mathbf{w}), \forall \mathbf{u}, \mathbf{v}, \mathbf{w} \in X
$$

and

$$
c_{T}(\mathbf{u}, T, r)=((\mathbf{u} \nabla) . T, r), \forall \mathbf{u} \in X, \forall T, r \in Y .
$$

Thus, we introduce the corresponding variational problem

Find $(\mathbf{u}, p) \in X \times M, T \in Y$ such that,

$$
\begin{array}{rlrl}
\frac{d}{d t}(\mathbf{u}(t), \mathbf{v})+(\nu(T(t)) \nabla \mathbf{u}(t), \nabla \mathbf{v})-(p(t), \operatorname{div} \mathbf{v})+c_{\mathbf{u}}(\mathbf{u}(t), \mathbf{u}(t), \mathbf{v}) & =(\mathbf{f}(t, T(t)), \mathbf{v}) & \forall \mathbf{v} \in X \\
\frac{d}{d t}(T(t), r)+(\alpha \nabla T(t) \nabla r)+c_{T}(\mathbf{u}(t), T(t), r) & =(g(t), r) & & \forall r \in Y, \\
(\operatorname{div} \mathbf{u}(t), q) & =0 & \forall q \in M, \\
\mathbf{u}(0) & =\mathbf{u}_{0}, & \text { in } \Omega, & \text { in } \Omega .
\end{array}
$$

Theorem 3.1. If the Assumption 2.4 holds, every solution of (E) verifies the bound

$$
\begin{aligned}
& \|\boldsymbol{u}\|_{L^{\infty}\left(0, \hat{T} ; L^{2}(\Omega)^{d}\right)}+\|\boldsymbol{u}\|_{L^{2}\left(0, \hat{T} ; H_{0}^{1}(\Omega)^{d}\right)}+\|T\|_{L^{\infty}\left(0, \hat{T} ; L_{0}^{2}(\Omega)\right)}+\|T\|_{L^{2}\left(0, \hat{T} ; H_{0}^{1}(\Omega)\right)} \\
& \quad \leq \hat{C}\left(\|g\|_{L^{2}\left(0, \hat{T} ; L^{2}(\Omega)\right)}+\left\|\mathbf{f}_{0}\right\|_{L^{2}\left(0, \hat{T} ; L^{2}(\Omega)^{d}\right)}+\left\|\boldsymbol{u}_{0}\right\|_{L^{2}(\Omega)^{d}}+\left\|T_{0}\right\|_{L^{2}(\Omega)}\right)
\end{aligned}
$$

where $\hat{C}$ is a positive constant which depends of $S_{2}^{0}, \hat{\nu}_{1}, \alpha$ and $c_{\mathbf{f}_{1}}$.

Proof. Let $(\mathbf{u}, p, T)$ be the solution of (E). To prove the bound (3.1), it suffices to choose $\mathbf{v}=\mathbf{u}$ in the first equation of problem (E) and use Assumption (2.4), Lemma 2.1 and Relation (2.4) for $\varepsilon=\frac{2}{\hat{\nu}_{1}}$ to get the bound:

$$
\frac{1}{2} \frac{d}{d t}\|\mathbf{u}(t)\|_{L^{2}(\Omega)^{d}}^{2}+\frac{\hat{\nu_{1}}}{2}|\mathbf{u}(t)|_{H_{0}^{1}(\Omega)^{d}}^{2} \leq \frac{\left(S_{2}^{0}\right)^{4} c_{\mathbf{f}_{1}}^{2}}{2 \hat{\nu}_{1}}|T(t)|_{H_{0}^{1}(\Omega)}^{2}+\frac{\left(S_{2}^{0}\right)^{2}}{\hat{\nu}_{1}}\left\|\mathbf{f}_{0}(t)\right\|_{L^{2}(\Omega)^{d}}^{2} .
$$

Next, we integrate with respect to $t$, between 0 and $\hat{T}$, to derive the following bound over $\mathbf{u}$ :

$$
\begin{aligned}
\frac{1}{\hat{\nu}_{1}}\|\mathbf{u}\|_{L^{\infty}\left(0, \hat{T} ; L^{2}(\Omega)^{d}\right)}+\|\mathbf{u}\|_{L^{2}\left(0, \hat{T} ; H_{0}^{1}(\Omega)^{d}\right) \leq}^{2} & \frac{\left(S_{2}^{0}\right)^{4} c_{\mathbf{f}_{1}}^{2}}{\left(\hat{\nu}_{1}\right)^{2}}\|T\|_{L^{2}\left(0, \hat{T} ; H_{0}^{1}(\Omega)\right)}^{2}+\frac{1}{\hat{\nu}_{1}}\left\|\mathbf{u}_{0}\right\|_{L^{2}(\Omega)^{d}}^{2} \\
& +\frac{\left(S_{2}^{0}\right)^{2}}{\hat{\nu}_{1}}\left\|\mathbf{f}_{0}\right\|_{L^{2}\left(0, \hat{T} ; L^{2}(\Omega)^{d}\right)}^{2} \cdot
\end{aligned}
$$

Similarly, we take the test function $r=T$ in the second equation of the problem (E). Then we integrate with respect to $t$ between 0 and $\hat{T}$ to obtain the inequality:

$$
\frac{1}{\alpha}\|T\|_{L^{\infty}\left(0, \hat{T} ; L^{2}(\Omega)\right)}+\|T\|_{L^{2}\left(0, \hat{T} ; H_{0}^{1}(\Omega)\right)}^{2} \leq\left(\frac{\left(S_{2}^{0}\right)^{2}}{\alpha^{2}}+\frac{1}{\alpha}\right)\left(\|g\|_{L^{2}\left(0, \hat{T} ; L^{2}(\Omega)\right)}^{2}+\left\|T_{0}\right\|_{L^{2}(\Omega)}^{2}\right) .
$$

Finally, we derive the result from (3.3) and (3.4).

A similar problem of (1.1) has been studied in [2] and discretized by using the spectral method where the data $\mathbf{f}$ is a given function independent of the heat $T\left(\mathbf{f}_{1}=\mathbf{0}\right)$. The authors showed the existence of the solution by using the Galerkin method and applying the Cauchy-Lipschitz theorem. The proof of 
the existence of the solution of (1.1) can be showed exactly by using the same method even where the function $\mathbf{f}$ depends of the temperature since $\mathbf{f}$ is a Lipcshitz-continuous function. Hence the results of the existence can be summarized in the following theorem.

Theorem 3.2. If the Assumption 2.4 holds, then Problem (E) admits at least one solution

$$
(\mathbf{u}, p, T) \in L^{2}\left(0, \hat{T} ; H_{0}^{1}(\Omega)^{d}\right) \times L^{2}\left(0, \hat{T} ; L_{0}^{2}(\Omega)\right) \times L^{2}\left(0, \hat{T} ; H_{0}^{1}(\Omega)\right) .
$$

Since the uniqueness for Navier-Stokes equations remains an open problem in dimension $d=3$, we only consider the case of dimension $d=2$ in the following theorem.

Theorem 3.3. Let $d=2$ and assume that $\nu$ is lipschitz-continuous, with Lipschitz constant $\nu^{*}$. If problem (E) admits a solution $(\mathbf{u}, p, T)$ which verifies:

$\mathbf{u} \in L^{p}\left(0, \hat{T} ; W^{1, r}(\Omega)^{2}\right)$ where $p \geq 4$ and $r \geq 4$, then this solution is unique.

Proof. Let $\left(\mathbf{u}_{1}, p_{1}, T_{1}\right)$ and $\left(\mathbf{u}_{2}, p_{2}, T_{2}\right)$ be two solutions of problem (E). In order to prove the uniqueness, we denote by $\mathbf{u}=\mathbf{u}_{1}-\mathbf{u}_{2}, p=p_{1}-p_{2}$ and $T=T_{1}-T_{2}$.

It follows from the second equation of (E), by adding and subtracting $c_{T}\left(\mathbf{u}_{2}, T_{1}, r\right)$ for $r=T$, the equation

$$
\int_{\Omega} \frac{\partial T}{\partial t}(\mathbf{x}, t) T(\mathbf{x}) d \mathbf{x}+\alpha(\nabla T(\mathbf{x}, t), \nabla T(\mathbf{x}))=-c_{T}\left(\mathbf{u}(t), T_{1}(t), T(t)\right) .
$$

Then, using Relation (2.2) for $\varepsilon=1$, Theorem 3.1 and again Relation (2.2), we get

$$
\begin{aligned}
& \frac{1}{2} \frac{d}{d t}\|T(t)\|_{L^{2}(\Omega)}^{2}+\alpha|T(t)|_{H_{0}^{1}(\Omega)}^{2} \leq \frac{1}{2}\left|T_{1}(t)\right|_{H_{0}^{1}(\Omega)}\left(\|\mathbf{u}(t)\|_{L^{4}(\Omega)^{2}}^{2}+\|T(t)\|_{L^{4}(\Omega)}^{2}\right) \\
& \leq \frac{1}{\sqrt{2}}\left(\frac{\varepsilon_{1}}{2}|\mathbf{u}(t)|_{H_{0}^{1}(\Omega)^{2}}^{2}+\frac{1}{2 \varepsilon_{1}}\|\mathbf{u}(t)\|_{L^{2}(\Omega)^{2}}^{2}\left|T_{1}(t)\right|_{H_{0}^{1}(\Omega)}^{2}\right) \\
& +\quad \frac{1}{\sqrt{2}}\left(\frac{\varepsilon_{2}}{2}|T(t)|_{H_{0}^{1}(\Omega)}^{2}+\frac{1}{2 \varepsilon_{2}}\left|T_{1}(t)\right|_{H_{0}^{1}(\Omega)}^{2}|| T(t) \|_{L^{2}(\Omega)}\right) .
\end{aligned}
$$

Similarly, we drive from the first equation of (E) that

$$
\begin{aligned}
& \frac{d}{d t}(\mathbf{u}(t), \mathbf{u}(t))+\left(\nu\left(T_{2}(t)\right) \nabla \mathbf{u}(t), \nabla \mathbf{u}(t)\right)=-c_{\mathbf{u}}\left(\mathbf{u}(t), \mathbf{u}_{1}(t), \mathbf{u}(t)\right) \\
& \quad+\left(\left(\nu\left(T_{2}(t)\right)-\nu\left(T_{1}(t)\right)\right) \nabla \mathbf{u}_{1}(t), \nabla \mathbf{u}(t)\right)+\left(\mathbf{f}_{1}\left(T_{1}(t)\right)-\mathbf{f}_{1}\left(T_{2}(t)\right), \mathbf{u}(t)\right) .
\end{aligned}
$$

We set $A=\left(\left(\nu\left(T_{2}\right)-\nu\left(T_{1}\right)\right) \nabla \mathbf{u}_{1}, \nabla \mathbf{u}\right)$. As $\mathbf{f}$ is $c_{\mathbf{f}_{1}}^{*}$-lipschitz and $\nu$ is bounded, then we obtain the following inequality:

$$
\frac{1}{2} \frac{d}{d t}\|\mathbf{u}(t)\|_{L^{2}(\Omega)^{2}}^{2}+\hat{\nu}_{1}|\mathbf{u}(t)|_{H_{0}^{1}(\Omega)^{2}}^{2} \leq|A|+\|\mathbf{u}(t)\|_{L^{4}(\Omega)^{2}}^{2}\left|\mathbf{u}_{1}(t)\right|_{H_{0}^{1}(\Omega)^{2}}+\left.c_{\mathbf{f}_{1}}^{*}\|T(t)\|\right|_{L^{2}(\Omega)}\|\mathbf{u}(t)\|_{L^{2}(\Omega)^{2}} .
$$

By applying Lemma 2.2 and Relation (2.4), we deduce the relation

$$
\begin{aligned}
& \frac{1}{2} \frac{d}{d t}\|\mathbf{u}(t)\|_{L^{2}(\Omega)^{2}}^{2}+\hat{\nu}_{1}|\mathbf{u}(t)|_{H_{0}^{1}(\Omega)^{2}}^{2} \\
& \quad \leq|A|+\frac{1}{2} \varepsilon_{3}|\mathbf{u}(t)|_{H_{0}^{1}(\Omega)^{2}}^{2}+\frac{1}{2 \varepsilon_{3}}\|\mathbf{u}(t)\|_{L^{2}(\Omega)^{2}}^{2}\left|\mathbf{u}_{1}(t)\right|_{H_{0}^{1}(\Omega)^{2}}^{2}+\frac{c_{\mathbf{f}_{1}}^{*}}{2}\left(\|\mathbf{u}(t)\|_{L^{2}(\Omega)^{2}}^{2}+\|T(t)\| L_{L^{2}(\Omega)}^{2}\right)
\end{aligned}
$$

Since the exact solution $\mathbf{u}_{1} \in L^{\infty}\left(0, \hat{T} ; W^{1, r}(\Omega)^{2}\right), r \geq 4$, so we obviously deduce the bound,

$$
|A| \leq \frac{\varepsilon_{4}}{2} \nu^{*}\left\|\mathbf{u}_{1}(t)\right\|_{W^{1, r}(\Omega)^{2}}|\mathbf{u}(t)|_{H_{0}^{1}(\Omega)^{2}}^{2}+\frac{1}{2 \varepsilon_{4}} \nu^{*}\left\|\mathbf{u}_{1}(t)\right\|_{W^{1, r}(\Omega)^{2}}\|T(t)\|_{L^{r^{*}}(\Omega)}^{2} .
$$

here $r^{*}=\frac{2 r}{r-2}$.

Since $r^{*} \leq 4$, we obtain by applying Relation (2.4),

$$
\begin{aligned}
|A| \leq & \frac{\varepsilon_{4}}{2} \nu^{*}\left\|\mathbf{u}_{1}(t)\right\|_{W^{1, r}(\Omega)^{2}}|\mathbf{u}(t)|_{H_{0}^{1}(\Omega)^{2}}^{2}+\frac{1}{2 \varepsilon_{4}} \nu^{*}\left\|\mathbf{u}_{1}(t)\right\|_{W^{1, r}(\Omega)^{2}}\left(\frac{1}{\sqrt{2} \varepsilon_{5}}\|T(t)\|_{L^{2}(\Omega)}^{2}\right. \\
& \left.+\frac{\varepsilon_{5}}{\sqrt{2}}|T(t)|_{H_{0}^{1}(\Omega)}^{2}\right)
\end{aligned}
$$


We consider the relations (3.5) and (3.7), choose $\varepsilon_{1}=\frac{\hat{\nu}_{1} \sqrt{2}}{3}, \varepsilon_{2}=\frac{\alpha}{4 \sqrt{2}}, \varepsilon_{3}=\frac{\hat{\nu}_{1}}{3}$,

$\varepsilon_{4}=\frac{\hat{\nu}_{1}}{3 \nu^{*}\left\|\mathbf{u}_{1}(t)\right\|_{W^{1, r}(\Omega)^{2}}}$, and $\varepsilon_{5}=\frac{\varepsilon_{4} \alpha}{\sqrt{2} \nu^{*}\left\|\mathbf{u}_{1}(t)\right\|_{W^{1, r}(\Omega)^{2}}}$ to obtain

$\frac{1}{2} \frac{d}{d t}\left(\|\mathbf{u}(t)\|_{L^{2}(\Omega)^{2}}^{2}+\|T(t)\|_{L^{2}(\Omega)}^{2}\right) \leq \hat{C}\left(\left\|\mathbf{u}_{1}(t)\right\|_{W^{1, r}(\Omega)^{2}}^{4}+\left|T_{1}(t)\right|_{H_{0}^{1}(\Omega)}^{2}+1\right)\left(\|\mathbf{u}(t)\|_{L^{2}(\Omega)^{2}}^{2}+\|T(t)\|_{L^{2}(\Omega)}^{2}\right)$.

We set $y(t)=\left(\|\mathbf{u}(t)\|_{L^{2}(\Omega)^{2}}^{2}+\|T(t)\|_{L^{2}(\Omega)}^{2}\right), \phi(t)=\hat{C}\left(\left\|\mathbf{u}_{1}(t)\right\|_{W^{1, r}(\Omega)^{2}}^{4}+\left|T_{1}(t)\right|_{H_{0}^{1}(\Omega)}^{2}+1\right)$ and integrate between 0 and $t$ to obtain $y(t) \leq \int_{0}^{t} \phi(s) y(s) d s$. By applying Gronwall's lemma, we obtain $y=0$, so that $\mathbf{u}=T=0$. Finally, the relation

$$
\int_{\Omega} \operatorname{div}(\mathbf{v}(\mathbf{x})) p(\mathbf{x}) d \mathbf{x}=0, \forall \mathbf{v} \in H_{0}^{1}(\Omega)^{2}
$$

gives $p=0$. Then we conclude the proof of the theorem.

\section{First ORDER DisCRETIZATION}

In this section, we propose first order time and space discretizations of the problem (E) in order to prove an a priori error estimation. First we use the semi-implicit Euler method for the time discretization and the finite element method for the space discretization. For the time discretization, we introduce a partition of the interval $[0, \hat{T}]$ into $N$ subintervals $\left[t_{n-1}, t_{n}\right]$ of length $\tau$ (the time step). For the space discretization, we suppose that the domain $\Omega$ is a polygon (respectively polyhedron) for $d=2$ (respectively $d=3$ ). Let $h>0$ be a discretization parameter in space and for each $h$, let $\mathcal{T}_{h}$ be a corresponding regular (or non-degenerate) family of triangles (respectively tetrahedra) for $d=2$ (respectively for $d=3$ ), in the usual sense that:

- $\bar{\Omega}$ is the union of all elements of $\mathcal{T}_{h}$;

- the intersection of two different elements of $\mathcal{T}_{h}$, if not empty, is a vertex or a whole edge (or a whole face of both of them for $d=3$ );

- the ratio of the diameter of an element $\kappa$ in $\mathcal{T}_{h}$ to the diameter of its inscribed sphere is bounded by a constant independent of $h$.

In what follows, $c, c^{\prime}, C, C^{\prime}, c_{1}, \ldots$ stand for generic constants which may vary from line to line but are always independent of $h$ and $\tau$.

Let $X_{h} \subset X$ and $M_{h} \subset M$ be a "stable" pair of finite-element spaces for discretizing the velocity $\mathbf{u}$ and the pressure $p$, stable in the sense that it satisfies a uniform discrete inf-sup condition: there exists a constant $\beta^{\star} \geq 0$, independent of $h$, such that

$$
\forall q_{h} \in M_{h}, \sup _{\mathbf{v}_{h} \in X_{h}} \frac{1}{\left|\mathbf{v}_{h}\right|_{H^{1}(\Omega) d}} \int_{\Omega} q_{h} \operatorname{div} \mathbf{v}_{h} d \mathbf{x} \geq \beta^{\star}\left\|q_{h}\right\|_{L^{2}(\Omega)} .
$$

Let $\mathbb{P}_{\kappa}$ denote the space of polynomials with total degree less than or equal to $\kappa$. We choose for the "mini-element" (see D. Arnold, F. Brezzi and M. Fortin in [4]), where in each element $\kappa$, the pressure $p$ is a polynomial of $\mathbb{P}_{1}$ and each component of the velocity is the sum of a polynomial of $\mathbb{P}_{1}$ and a "bubble" function $b_{\kappa}$ (for each element $\kappa$, the bubble function is equal to the product of the barycentric coordinates associated with the vertices of $\kappa$ ).

We introduce the following finite-element space

$$
Z_{h}=\left\{q_{h} \in C^{0}(\bar{\Omega}) ; \forall \kappa \in \mathcal{T}_{h}, q_{\left.h\right|_{\kappa}} \in \mathbb{P}_{1}\right\},
$$

where $h$ denotes the maximal diameter of the elements of $\mathcal{T}_{h}$.

Therefore, the finite-element spaces for the velocity and the pressure are :

$$
\begin{gathered}
X_{h}=\left\{\mathbf{v}_{h} \in C^{0}(\bar{\Omega})^{d} ; \forall \kappa \in \mathcal{T}_{h}, \mathbf{v}_{\left.h\right|_{\kappa}} \in \mathcal{P}(\kappa), \mathbf{v}_{\left.h\right|_{\partial \Omega}}=0\right\}, \\
M_{h}=\left\{q_{h} \in Z_{h}, \int_{\Omega} q_{h} d \mathbf{x}=0\right\}
\end{gathered}
$$


where

$$
\mathcal{P}(\kappa)=\left[\mathbb{P}_{1} \oplus \operatorname{Span}\left(b_{\kappa}\right)\right]^{d} .
$$

Furthermore, the finite element space for the temperature is

$$
Y_{h}=\left\{\theta_{h} \in Z_{h}, \theta_{\left.h\right|_{\partial \Omega}}=0\right\} .
$$

There exists an approximation operator $P_{h} \in \mathcal{L}\left(H_{0}^{1}(\Omega)^{d} ; X_{h}\right)$ such that (see V. Girault and P.-A. Raviart in $[7])$ :

$$
\forall v \in H_{0}^{1}(\Omega)^{d}, \quad \forall q_{h} \in M_{h}, \quad \int_{\Omega} q_{h} \operatorname{div}\left(P_{h}(v)-v\right) d \mathbf{x}=0
$$

and for $k=0$ or 1 ,

$$
\forall v \in\left[H^{1+k}(\Omega) \cap H_{0}^{1}(\Omega)\right]^{d}, \quad\left\|P_{h}(v)-v\right\|_{L^{2}(\Omega)^{d}} \leq C_{1} h^{1+k}|v|_{H^{1+k}(\Omega)^{d}},
$$

and forall $r \geq 2, k=0$ or 1 ,

$$
\forall v \in\left[W^{1+k, r}(\Omega) \cap H_{0}^{1}(\Omega)\right]^{d}, \quad\left|P_{h}(v)-v\right|_{W^{1, r}(\Omega)^{d}} \leq C_{2} h^{k}|v|_{W^{1+k, r}(\Omega)^{d}} .
$$

In addition, there exists an approximation operator (when $d=2$, see Bernardi and Girault [5] or Clément [9]; when $d=2$ or $d=3$, see Scott and Zhang [12]), $R_{h}$ in $\mathcal{L}\left(W^{1, p}(\Omega) ; Z_{h}\right)$ and in $\mathcal{L}\left(W^{1, p}(\Omega) \cap\right.$ $\left.H_{0}^{1}(\Omega) ; Y_{h}\right)$ such that for $m=0,1, l=0,1$, and all $p \geq 2$,

$$
\forall S \in W^{l+1, p}(\Omega),\left|S-R_{h}(S)\right|_{W^{m, p}(\Omega)} \leq C_{3} h^{l+1-m}|S|_{W^{l+1, p}(\Omega)},
$$

Regarding the pressure, an easy modification of $R_{h}$ yields an operator $r_{h}$ in $\mathcal{L}\left(H^{1}(\Omega) ; Z_{h}\right)$ and in $\mathcal{L}\left(H^{1}(\Omega) \cap L_{0}^{2}(\Omega) ; M_{h}\right)$ (see for instance Abboud, Girault and Sayah [1]), satisfying (4.9). In order to introduce the discrete scheme, we define the following forms: for all $\mathbf{u}_{h}, \mathbf{v}_{h}, \mathbf{w}_{h} \in X_{h}$, and $T_{h}, r_{h} \in Y_{h}$

$$
\begin{array}{r}
d_{\mathbf{u}}\left(\mathbf{u}_{h}, \mathbf{v}_{h}, \mathbf{w}_{\mathbf{h}}\right)=c_{\mathbf{u}}\left(\mathbf{u}_{h}, \mathbf{v}_{h}, \mathbf{w}_{h}\right)+\frac{1}{2}\left(\operatorname{div}\left(\mathbf{u}_{h}\right) \mathbf{v}_{h}, \mathbf{w}_{h}\right), \\
d_{T}\left(\mathbf{u}_{h}, T_{h}, r_{h}\right)=c_{T}\left(\mathbf{u}_{h}, T_{h}, r_{h}\right)+\frac{1}{2}\left(\operatorname{div}\left(\mathbf{u}_{h}\right) T_{h}, r_{h}\right) .
\end{array}
$$

The first discrete problem corresponding to $(E)$ is:

for every $n \in\{1, \ldots, N\}$, knowing $\mathbf{u}_{h}^{n-1} \in X_{h}, T_{h}^{n-1} \in Y_{h}$, we compute $\mathbf{u}_{h}^{n} \in X_{h}, p_{h}^{n} \in M_{h}, T_{h}^{n} \in Y_{h}$ such that for all $\mathbf{v}_{h} \in X_{h}, r_{h} \in Y_{h}$ and $q_{h} \in M_{h}$,

$$
\begin{aligned}
& \frac{1}{\tau}\left(\mathbf{u}_{h}^{n}-\mathbf{u}_{h}^{n-1}, \mathbf{v}_{h}\right)+\left(\nu\left(T_{h}^{n-1}\right) \nabla \mathbf{u}_{h}^{n}, \nabla \mathbf{v}_{h}\right)-\left(p_{h}^{n}, \operatorname{div} \mathbf{v}_{h}\right)+d_{\mathbf{u}}\left(\mathbf{u}_{h}^{n-1}, \mathbf{u}_{h}^{n}, \mathbf{v}_{h}\right)=\left(\mathbf{f}^{n}\left(T_{h}^{n-1}\right), \mathbf{v}_{h}\right), \\
& \frac{1}{\tau}\left(T_{h}^{n}-T_{h}^{n-1}, r_{h}\right)+\alpha\left(\nabla T_{h}^{n}, \nabla r_{h}\right)+d_{T}\left(\mathbf{u}_{h}^{n}, T_{h}^{n}, r_{h}\right)=\left(g^{n}, r_{h}\right) \\
& \left(q_{h}, \operatorname{div} \mathbf{u}_{h}^{n}\right)=0
\end{aligned}
$$

where $\mathbf{u}_{h}^{0}$ and $T_{h}^{0}$ are the approximations of $\mathbf{u}_{0}$ and $T_{0}, g^{n}$ and $\mathbf{f}^{n}\left(T_{h}^{n-1}\right)$ are given as

$$
\begin{aligned}
g^{n} & =\frac{1}{\tau} \int_{t_{n-1}}^{t_{n}} g(s) d s, \quad \text { where } \quad \mathbf{f}_{0}^{n}=\frac{1}{\tau} \int_{t_{n-1}}^{t_{n}} \mathbf{f}_{0}(s) d s . \\
\mathbf{f}^{n}\left(T_{h}^{n-1}\right) & =\mathbf{f}_{0}^{n}+\mathbf{f}_{1}\left(T_{h}^{n-1}\right), \quad
\end{aligned}
$$

The second discrete problem associated with Problem $(E)$ is the following: for every $n \in\{1, \ldots, N\}$, knowing $\mathbf{u}_{h}^{n-1} \in X_{h}, T_{h}^{n-1} \in Y_{h}$, we compute $\mathbf{u}_{h}^{n} \in X_{h}, p_{h}^{n} \in M_{h}, T_{h}^{n} \in Y_{h}$ such that for all $\mathbf{v}_{h} \in X_{h}, r_{h} \in Y_{h}, q_{h} \in M_{h}$,

$$
\begin{aligned}
& \frac{1}{\tau}\left(\mathbf{u}_{h}^{n}-\mathbf{u}_{h}^{n-1}, \mathbf{v}_{h}\right)+\left(\nu\left(T_{h}^{n-1}\right) \nabla \mathbf{u}_{h}^{n}, \nabla \mathbf{v}_{h}\right)-\left(p_{h}^{n}, \operatorname{div} \mathbf{v}_{h}\right)+c_{\mathbf{u}}\left(\mathbf{u}_{h}^{n-1}, \mathbf{u}_{h}^{n}, \mathbf{v}_{h}\right)=\left(\mathbf{f}^{n}\left(T_{h}^{n-1}\right), \mathbf{v}_{h}\right), \\
& \frac{1}{\tau}\left(T_{h}^{n}-T_{h}^{n-1}, r_{h}\right)+\alpha\left(\nabla T_{h}^{n}, \nabla r_{h}\right)+c_{T}\left(\mathbf{u}_{h}^{n}, T_{h}^{n}, r_{h}\right)=\left(g^{n}, r_{h}\right) \\
& \left(q_{h}, \operatorname{div} \mathbf{u}_{h}^{n}\right)=0
\end{aligned}
$$

Remark 4.1. For the simplicity of the establishment of the a priori error estimates, we consider from now on $T_{h}^{0}=R_{h} T(0)$ and $\mathbf{u}_{h}^{0}=P_{h} \mathbf{u}(0)$. 
Remark 4.2. The difference between the previous numerical schemes (Eds1) and (Eds2) is the trilinear forms; in the first one they are $d_{\mathbf{u}}$ and $d_{T}$ and in the second one $c_{\mathbf{u}}$ and $c_{T}$. In both schemes, we compute $\left(\mathbf{u}_{h}^{n}, p_{h}^{n}\right)$ and then, use the computed $\mathbf{u}_{h}^{n}$ to calculate $T_{h}^{n}$. The main differences between the (Eds1) and (Eds2) are:

(1) (Eds1) contains two supplementary terms provided from the definition of the forms $\left(d_{\mathbf{u}}, d_{T}\right)$ and $\left(c_{\mathbf{u}}, c_{T}\right)$, so the CPU time of computation of (Eds2) is less than that of (Eds1).

(2) The studies of (Eds1) is more simple than (Eds2) due to the stability relations

$$
d_{\mathbf{u}}\left(\mathbf{u}_{h}, \mathbf{v}_{h}, \mathbf{v}_{h}\right)=d_{T}\left(\mathbf{u}_{h}, T_{h}, T_{h}\right)=0, \quad \forall \mathbf{u}_{h}, \mathbf{v}_{h} \in X_{h}, \forall T_{h} \in Y_{h} .
$$

This last relations aren't valid for the forms $c_{\mathbf{u}}$ and $c_{T}$.

Remark 4.3. In fact, for the simplicity we shall study in this paper the properties of the first scheme (Eds1). The results corresponding to the second scheme (Eds2) are obtained by using the same method but by applying the following bounds (see [8]):

For every $\mathbf{u}_{h} \in X_{h}, \mathbf{v}_{h} \in X_{h}, T_{h} \in Y_{h}$, there exists two constants $\hat{C}_{\mathbf{u}}$ and $\hat{C}_{T}$ independent of $h$ such that

$$
\left|c_{\mathbf{u}}\left(\mathbf{u}_{h}^{n-1}, \mathbf{u}_{h}, \mathbf{u}_{h}\right)\right| \leq \hat{C}_{\mathbf{u}} h^{r}\left\|\operatorname{div} \mathbf{u}_{h}^{n-1}\right\|_{L^{2}(\Omega)}\left|\mathbf{u}_{h}\right|_{H^{1}(\Omega)^{d}}^{2}
$$

and

$$
\left|c_{T}\left(\mathbf{u}_{h}^{n}, T_{h}, T_{h}\right)\right| \leq \hat{C}_{T} h^{r}\left\|\operatorname{div} \mathbf{u}_{h}^{n}\right\|_{L^{2}(\Omega)}\left|T_{h}\right|_{H^{1}(\Omega)}^{2}
$$

where $r=1-\hat{\varepsilon}, \hat{\varepsilon}>0$ for $d=2$ and $r=\frac{1}{2}$ for $d=3$.

The relations (4.12) and (4.13) allow to get the existence and uniqueness of the numerical solution of (Eds2) where the space step $h$ is smaller than a given real positive number $h_{0}$ (see [8] for details).

Theorem 4.4. (Existence and uniqueness of the solution of (Eds1))

At each time step $n$, for a given $\boldsymbol{u}_{h}^{n-1} \in X_{h}, T_{h}^{n-1} \in Y_{h}$, under the assumption 2.4, Problem (Eds1) admits a unique solution $\left(\boldsymbol{u}_{h}^{n}, p_{h}^{n}, T_{h}^{n}\right) \in X_{h} \times M_{h} \times Y_{h}$ which verifies, for $m=1, \ldots, N$, the following bounds

$$
\begin{aligned}
\frac{1}{2}\left\|\boldsymbol{u}_{h}^{m}\right\|_{L^{2}(\Omega)^{2}}^{2}+\sum_{n=0}^{m} \frac{\hat{\nu}_{1} \tau}{2}\left|\boldsymbol{u}_{h}^{n}\right|_{H_{0}^{1}(\Omega)^{d}}^{2} \leq & \tilde{C}_{d}\left(\|g\|_{L^{2}\left(0, \hat{T}, L^{2}(\Omega)\right)}^{2}+\left\|\mathbf{f}_{0}\right\|_{L^{2}\left(0, \hat{T}, L^{2}(\Omega)^{d}\right)}^{2}\right. \\
& \left.+\left\|T_{h}^{0}\right\|_{L^{2}(\Omega)}^{2}+\left\|\boldsymbol{u}_{h}^{0}\right\|_{L^{2}(\Omega)^{d}}^{2}\right) \\
\frac{1}{2}\left\|T_{h}^{m}\right\|_{L^{2}(\Omega)}^{2}+\sum_{n=0}^{m} \frac{\alpha}{2} \tau\left|T_{h}^{n}\right|_{H_{0}^{1}(\Omega)}^{2} \leq & \tilde{C}_{d}^{\prime}\left(\|g\|_{L^{2}\left(0, \hat{T}, L^{2}(\Omega)\right)}^{2}+\left\|T_{h}^{0}\right\|_{L^{2}(\Omega)}^{2}\right),
\end{aligned}
$$

where $\tilde{C}_{d}$ and $\tilde{C}_{d}^{\prime}$ are positive constants independent of $h$ and $m$.

Proof. For $\mathbf{u}_{h}^{n-1} \in X_{h}$, it is clear that the first and third equations of Problem (Eds1) admit a unique solution $\left(\mathbf{u}_{h}^{n}, p_{h}^{n}\right)$ as a consequence of the relation (4.11), the coerciveness of the corresponding bilinear form on $X_{h} \times X_{h}$ and the inf-sup condition (4.1). Thus, knowing $\mathbf{u}_{h}^{n} \in X_{h}$ and $T_{h}^{n-1} \in Y_{h}$, the second equation of Problem (Eds1) also admits a unique solution $T_{h}^{n} \in Y_{h}$ as a consequence of the relation (4.11) and the coerciveness of the corresponding bilinear form on $Y_{h} \times Y_{h}$. Therefore, by taking $\mathbf{v}_{h}=\mathbf{u}_{h}^{n}$ in the first equation and $r_{h}=T_{h}^{n}$ in the second equation of Problem (Eds1), we get, due to Formulas (2.5) and (2.4) (for $\varepsilon=\frac{2}{\hat{\nu}_{1}}$ ), and Assumption 2.4 :

$$
\left\|\mathbf{u}_{h}^{n}\right\|_{L^{2}(\Omega)^{d}}^{2}-\left\|\mathbf{u}_{h}^{n-1}\right\|_{L^{2}(\Omega)^{d}}^{2}+\frac{\hat{\nu}_{1}}{2} \tau\left|\mathbf{u}_{h}^{n}\right|_{H_{0}^{1}(\Omega)^{d}}^{2} \leq \frac{\left(S_{2}^{0}\right)^{2} c_{\mathbf{f}}^{2}}{\hat{\nu}_{1}} \tau\left\|T_{h}^{n-1}\right\|_{L^{2}(\Omega)^{d}}^{2}+\frac{\left(S_{2}^{0}\right)^{2}}{\hat{\nu}_{1}} \tau\left\|\mathbf{f}_{0}^{n}\right\|_{L^{2}(\Omega)^{d}}^{2}
$$

and

$$
\left\|T_{h}^{n}\right\|_{L^{2}(\Omega)}^{2}-\left\|T_{h}^{n-1}\right\|_{L^{2}(\Omega)}^{2}+\frac{\alpha}{2} \tau\left|T_{h}^{n}\right|_{H^{1}(\Omega)}^{2} \leq \frac{\left(S_{2}^{0}\right)^{2}}{\alpha} \tau\left\|g^{n}\right\|_{L^{2}(\Omega)}^{2} .
$$

We sum over $n=1, \ldots, m$ and we obtain the results. 
Theorem 4.5. Let $(\boldsymbol{u}, T, p)$ be the solution of Problem $E$ and $\left(\boldsymbol{u}_{h}^{n}, T_{h}^{n}, p_{h}^{n}\right)$ be the solution of Problem (Eds1). If $\boldsymbol{u} \in L^{\infty}\left(0, \hat{T} ; H^{1}(\Omega)^{2}\right) \cap L^{2}\left(0, \hat{T} ; H^{2}(\Omega)^{2}\right), \boldsymbol{u}^{\prime} \in L^{2}\left(0, \hat{T} ; H^{1}(\Omega)^{2}\right), T \in L^{\infty}\left(0, \hat{T} ; H^{1}(\Omega)\right) \cap$ $L^{2}\left(0, \hat{T} ; H^{2}(\Omega)\right)$ and $T^{\prime} \in L^{\infty}\left(0, \hat{T} ; H^{1}(\Omega)\right)$, there exists positive constants $C_{T}^{\prime}$ and $C_{T}^{\prime \prime}$ depending on $\boldsymbol{u}, \boldsymbol{u}^{\prime}, T, T^{\prime}$ and $\alpha$ such that, for all $m \leq N$, we have

$$
\begin{gathered}
\frac{1}{2}\left\|T_{h}^{m}-R_{h} T\left(t_{m}\right)\right\|_{L^{2}(\Omega)}^{2}+\frac{1}{2} \sum_{n=1}^{m} \alpha \tau\left|T_{h}^{n}-R_{h} T\left(t_{n}\right)\right|_{H^{1}(\Omega)}^{2}+\frac{1}{2} \sum_{n=1}^{m}\left\|T_{h}^{n}-R_{h} T\left(t_{n}\right)-\left(T_{h}^{n-1}-R_{h} T\left(t_{n-1}\right)\right)\right\|_{L^{2}(\Omega)}^{2} \\
\leq C_{T^{\prime}}\left(h^{2}+\tau^{2}\right)+C_{T}^{\prime \prime} \sum_{n=1}^{m} \tau\left|\mathbf{u}_{h}^{n}-P_{h} \mathbf{u}\left(t_{n}\right)\right|_{H^{1}(\Omega)^{d}} .
\end{gathered}
$$

Proof. We choose the test function $r=r_{h}^{n}=T_{h}^{n}-R_{h} T\left(t_{n}\right)$ in the second equation of (E). Then, we integrate it over $\left[t_{n-1}, t_{n}\right]$, substract the second equation of (Eds1) multiplied by the time step $\tau$, insert $\pm R_{h} T\left(t_{n-1}\right)$ and $\pm R_{h} T\left(t_{n}\right)$. We obtain :

$$
\begin{array}{r}
\frac{1}{2}\left\|r_{h}^{n}\right\|_{L^{2}(\Omega)}^{2}-\frac{1}{2}\left\|r_{h}^{n-1}\right\|_{L^{2}(\Omega)}^{2}+\frac{1}{2}\left\|r_{h}^{n}-r_{h}^{n-1}\right\|_{L^{2}(\Omega)}^{2}+\alpha \tau\left|r_{h}^{n}\right|_{H^{1}(\Omega)}^{2}=-\int_{t_{n-1}}^{t_{n}}\left(R_{h} T^{\prime}(t)-T^{\prime}(t), r_{h}^{n}\right) d t \\
+\alpha \int_{t_{n-1}}^{t_{n}}\left(\nabla\left(T(t)-R_{h} T\left(t_{n}\right)\right), \nabla r_{h}^{n}\right) d t+\int_{t_{n-1}}^{t_{n}}\left((\mathbf{u}(t) \nabla) \cdot T(t)-\left(\mathbf{u}_{h}^{n} \nabla\right) \cdot T_{h}^{n}, r_{h}^{n}\right) d t-\frac{\tau}{2}\left(\operatorname{div} \mathbf{u}_{h}^{n} T_{h}^{n}, r_{h}^{n}\right)
\end{array}
$$

The first term of the right hand side of (4.18) can be bounded, for any $\tilde{\varepsilon}_{1}>0$, as follows:

$$
\left|\int_{t_{n-1}}^{t_{n}}\left(R_{h} T^{\prime}(t)-T^{\prime}(t), r_{h}^{n}\right) d t\right| \leq \frac{c_{1}^{2} h^{2}}{2 \tilde{\varepsilon}_{1}}\left\|T^{\prime}\right\|_{L^{2}\left(t_{n-1}, t_{n} ; H^{1}(\Omega)\right)}^{2}+\frac{\tau\left(S_{2}^{0}\right)^{2} \tilde{\varepsilon}_{1}}{2}\left\|r_{h}^{n}\right\|_{H^{1}(\Omega)}^{2} .
$$

By inserting $\pm R_{h} T(t)$, the second term of the right hand side of (4.18) can be treated as:

$$
\begin{aligned}
\alpha\left|\int_{t_{n-1}}^{t_{n}}\left(\nabla\left(R_{h} T\left(t_{n}\right)-T(t)\right), \nabla r_{h}^{n}\right) d t\right| \leq & \alpha\left|\int_{t_{n-1}}^{t_{n}} \int_{t}^{t_{n}}\left(\nabla\left(R_{h} T^{\prime}(t)\right), \nabla r_{h}^{n}\right) d t\right| \\
& +\alpha\left|\int_{t_{n-1}}^{t_{n}}\left(\nabla\left(R_{h} T-T\right)(t), r_{h}^{n}\right) d s d t\right|,
\end{aligned}
$$

where, for any $\tilde{\varepsilon}_{2}>0$ and $\tilde{\varepsilon}_{3}>0$, we have

$$
\begin{aligned}
\left|\int_{t_{n-1}}^{t_{n}} \int_{t}^{t_{n}}\left(\nabla\left(R_{h} T^{\prime}(t)\right), \nabla r_{h}^{n}\right) d t\right| & \leq \int_{t_{n-1}}^{t_{n}} \int_{t_{n-1}}^{s}\left|R_{h} T^{\prime}(s)\right|_{H^{1}(\Omega)}\left|r_{h}^{n}\right|_{H^{1}(\Omega)} d t d s \\
& \leq c_{2}\left|r_{h}^{n}\right|_{H^{1}(\Omega)} \int_{t_{n-1}}^{t_{n}}\left|T^{\prime}(s)\right|_{H^{1}(\Omega)}\left(s-t_{n-1}\right) d s \\
& \leq \frac{\tau^{2} c_{2}^{2}}{2 \sqrt{3} \tilde{\varepsilon}_{2}}\left\|T^{\prime}\right\|_{L^{2}\left(t_{n-1}, t_{n} ; H^{1}(\Omega)\right)}^{2}+\frac{\tilde{\varepsilon}_{2} \tau}{2 \sqrt{3}}\left|r_{h}^{n}\right|_{H^{1}(\Omega)}^{2},
\end{aligned}
$$

and

$$
\left|\int_{t_{n-1}}^{t_{n}}\left(\nabla\left(R_{h} T-T\right)(t), r_{h}^{n}\right) d s d t\right| \leq \frac{c_{3}^{2} h^{2}}{2 \tilde{\varepsilon}_{3}}\|T\|_{L^{2}\left(t_{n-1}, t_{n} ; H^{2}(\Omega)\right)}^{2}+\frac{\tau \tilde{\varepsilon}_{3}}{2}\left|r_{h}^{n}\right|_{H^{1}(\Omega)}^{2} .
$$

Now, we have to treat the nonlinear term in (4.18) denoted by $b_{n}$. By inserting $\pm \tau\left(\left(\mathbf{u}_{h}^{n} \nabla\right) R_{h} T\left(t_{n}\right), r_{h}^{n}\right)$ and $\pm \frac{\tau}{2}\left(\operatorname{div}\left(\mathbf{u}_{h}^{n}\right) R_{h} T\left(t_{n}\right), r_{h}^{n}\right), b_{n}$ becomes by using (4.11) :

$$
\left.b_{n}=\tau\left(\left(\mathbf{u}_{h}^{n} \nabla\right) \cdot R_{h} T\left(t_{n}\right), r_{h}^{n}\right)+\frac{\tau}{2}\left(\operatorname{div}\left(\mathbf{u}_{h}^{n}\right) R_{h} T\left(t_{n}\right)\right), r_{h}^{n}\right)-\int_{t_{n-1}}^{t_{n}}\left((\mathbf{u}(t) \nabla) \cdot T(t), r_{h}^{n}\right) d t .
$$


Furthermore, by inserting $\pm \tau\left(P_{h} \mathbf{u}\left(t_{n}\right) \nabla R_{h} T\left(t_{n}\right), r_{h}^{n}\right), \pm \frac{\tau}{2}\left(\operatorname{div}\left(P_{h} \mathbf{u}\left(t_{n}\right)\right) R_{h} T\left(t_{n}\right), r_{h}^{n}\right), \pm \int_{t_{n-1}}^{t_{n}}\left(\left(P_{h} \mathbf{u}\left(t_{n}\right) \nabla\right) \cdot T(t), r_{h}^{n}\right) d t$, and $\pm \frac{1}{2} \int_{t_{n-1}}^{t_{n}}\left(\operatorname{div} P_{h} \mathbf{u}\left(t_{n}\right) T(t), r_{h}^{n}\right) d t$, we obtain

$$
\begin{aligned}
b_{n}= & \tau\left(\left(\left(\mathbf{u}_{h}^{n}-P_{h} \mathbf{u}\left(t_{n}\right)\right) \nabla\right) \cdot R_{h} T\left(t_{n}\right), r_{h}^{n}\right)+\int_{t_{n-1}}^{t_{n}}\left(\left(P_{h} \mathbf{u}\left(t_{n}\right) \nabla\right) \cdot\left(R_{h} T\left(t_{n}\right)-T(t)\right), r_{h}^{n}\right) d t \\
& +\int_{t_{n-1}}^{t_{n}}\left(\left(\left(P_{h} \mathbf{u}\left(t_{n}\right)-\mathbf{u}(t)\right) \nabla\right) \cdot T(t), r_{h}^{n}\right) d t+\frac{\tau}{2}\left(\operatorname{div}\left(\mathbf{u}_{h}^{n}-P_{h} \mathbf{u}\left(t_{n}\right)\right) R_{h} T\left(t_{n}\right), r_{h}^{n}\right) \\
& +\frac{1}{2} \int_{t_{n-1}}^{t_{n}}\left(\operatorname{div}\left(P_{h} \mathbf{u}\left(t_{n}\right)\right)\left(R_{h} T\left(t_{n}\right)-T(t)\right), r_{h}^{n}\right) d t+\frac{1}{2} \int_{t_{n-1}}^{t_{n}}\left(\operatorname{div}\left(P_{h} \mathbf{u}\left(t_{n}\right)-\mathbf{u}(t)\right) T(t), r_{h}^{n}\right) d t .
\end{aligned}
$$

We denote by $b_{n, 1}, \ldots, b_{n, 6}$ the terms of the right hand side of (4.19) which can be bounded as following: for every positive real number $\tilde{\varepsilon}_{4}>0$ we have:

$$
\begin{aligned}
\left|b_{n, 1}\right| & \leq \int_{t_{n-1}}^{t_{n}}\left\|\mathbf{u}_{h}^{n}-P_{h} \mathbf{u}\left(t_{n}\right)\right\|_{L^{4}(\Omega)^{d}}\left|R_{h} T\left(t_{n}\right)\right|_{H^{1}(\Omega)}\left\|r_{h}^{n}\right\|_{L^{4}(\Omega)} d t \\
& \leq \frac{c_{4}\left(S_{4}^{0}\right)^{4}}{2 \tilde{\varepsilon}_{4}} \tau\|T\|_{L^{\infty}\left(0, \hat{T} ; H^{1}(\Omega)\right)}^{2}\left|\mathbf{u}_{h}^{n}-P_{h} \mathbf{u}\left(t_{n}\right)\right|_{H^{1}(\Omega)^{d}}^{2}+\frac{\tilde{\varepsilon}_{4} \tau}{2}\left|r_{h}^{n}\right|_{H^{1}(\Omega)},
\end{aligned}
$$

For every positive real number $\tilde{\varepsilon}_{5}$ and $\tilde{\varepsilon}_{6}$, we insert $\pm \int_{t_{n-1}}^{t_{n}}\left(\left(P_{h} u\left(t_{n}\right) \nabla R_{h} T(t), r_{h}^{n}\right) d t\right.$ in $b_{n, 2}$ and obtain:

$$
\begin{aligned}
\left|b_{3,2}\right| \leq & \left|\int_{t_{n-1}}^{t_{n}} \int_{t}^{t_{n}}\left(\left(P_{h} \mathbf{u}\left(t_{n}\right) \nabla\right) \cdot R_{h} T^{\prime}(s), r_{h}^{n}\right) d s d t\right|+\left|\int_{t_{n-1}}^{t_{n}}\left(\left(P_{h} \mathbf{u}\left(t_{n}\right) \nabla\right) \cdot\left(R_{h} T-T\right)(t), r_{h}^{n}\right) d t\right| \\
\leq & \frac{c_{5}\left(S_{4}^{0}\right)^{4}}{2}\|\mathbf{u}\|_{L^{\infty}\left(0, \hat{T} ; H^{1}(\Omega)^{d}\right)}^{2}\left(\frac{\tau^{2}}{\tilde{\varepsilon}_{5}}\left\|T^{\prime}\right\|_{L^{\infty}\left(0, \hat{T} ; H^{1}(\Omega)\right)}^{2}+\frac{h^{2}}{\tilde{\varepsilon}_{6}}\|T\|_{L^{2}\left(0, \hat{T} ; H^{2}(\Omega)\right)}^{2}\right) \\
& +\frac{\tilde{\varepsilon}_{5}+\tilde{\varepsilon}_{6}}{2} \tau\left|r_{h}^{n}\right|_{H^{1}(\Omega)}^{2} .
\end{aligned}
$$

We insert $\pm \int_{t_{n-1}}^{t_{n}}\left(\left(P_{h} \mathbf{u}(t) \nabla\right) \cdot T(t), r_{h}^{n}\right) d t$ in $b_{n, 3}$ and obtain for every positive real number $\tilde{\varepsilon}_{7}$ and $\tilde{\varepsilon}_{8}$,

$$
\begin{aligned}
\left|b_{n, 3}\right| \leq & \left(S_{4}^{0}\right)^{2} \int_{t_{n-1}}^{t_{n}}\left(\int_{t}^{t_{n}}\left|P_{h} \mathbf{u}^{\prime}(s)\right|_{H^{1}(\Omega)^{d}} d s+\left|\left(P_{h} \mathbf{u}-\mathbf{u}\right)(t)\right|_{H^{1}(\Omega)^{d}}\right)|T(t)|_{H^{1}(\Omega)}\left|r_{h}^{n}\right|_{H^{1}(\Omega)} d t \\
\leq & \frac{\left(S_{4}^{0}\right)^{4} c_{7}}{2}\|T\|_{L^{\infty}\left(0, \hat{T} ; H^{1}(\Omega)\right)}^{2}\left(\frac{1}{\tilde{\varepsilon}_{7}} \tau^{2}\left\|\mathbf{u}^{\prime}\right\|_{L^{2}\left(0, \hat{T} ; H^{1}(\Omega)^{d}\right)}^{2}+\frac{1}{\tilde{\varepsilon}_{8}} h^{2}\|\mathbf{u}\|_{L^{2}\left(0, \hat{T} ; H^{2}(\Omega)^{d}\right)}^{2}\right) \\
& +\frac{\tilde{\varepsilon}_{7}+\tilde{\varepsilon}_{8}}{2} \tau\left|r_{h}^{n}\right|_{H^{1}(\Omega)}^{2} .
\end{aligned}
$$

The term $b_{n, 4}$ can be bounded for every positive real number $\varepsilon_{9}$ as:

$$
\left|b_{n, 4}\right| \leq \frac{c_{9}}{2 \varepsilon_{9}} \tau\left(S_{4}^{0}\right)^{4}\|T\|_{L^{\infty}\left(0, \hat{T} ; H^{1}(\Omega)\right)}^{2}\left|\mathbf{u}_{h}^{n}-P_{h} \mathbf{u}\left(t_{n}\right)\right|_{H^{1}(\Omega)^{d}}^{2}+\frac{1}{2} \varepsilon_{9} \tau\left|r_{h}^{n}\right|_{H^{1}(\Omega)}^{2} .
$$

Furthermore, the terms $b_{n, 5}$ and $b_{n, 6}$ can be treated exactly like $b_{n, 2}$ and $b_{n, 3}$ and we get for every positive real numbers $\varepsilon_{10}, \varepsilon_{11}, \varepsilon_{12}$ and $\varepsilon_{13}$,

$$
\begin{aligned}
\left|b_{n, 5}\right| \leq & \left.\frac{c_{10}\left(S_{4}^{0}\right)^{4}}{2}\|\mathbf{u}\|_{L^{\infty}\left(0, \hat{T} ; H^{1}(\Omega)^{d}\right)}^{2}\left(\frac{\tau^{2}}{\tilde{\varepsilon}_{10}}\left\|T^{\prime}\right\|_{L^{2}\left(0, \hat{T} ; H^{1}(\Omega)\right)}^{2}+h^{2} \tilde{\varepsilon}_{11}\|\mathbf{u}\|_{L^{\infty}\left(0, \hat{T} ; H^{1}(\Omega)^{d}\right)}^{2}\right)\right) \\
& +\frac{\tilde{\varepsilon}_{10}+\tilde{\varepsilon}_{11}}{2} \tau\left|r_{h}^{n}\right|_{H^{1}(\Omega)}^{2}
\end{aligned}
$$

and

$$
\begin{aligned}
\left|b_{n, 6}\right| \leq & \frac{c_{12}\left(S_{4}^{0}\right)^{4}}{2}\|T\|_{L^{\infty}\left(0, \hat{T} ; H^{1}(\Omega)\right)}^{2}\left(\frac{\tau^{2}}{\tilde{\varepsilon}_{12}}\left\|\mathbf{u}^{\prime}\right\|_{L^{2}\left(0, \hat{T} ; H^{1}(\Omega)^{d}\right)}^{2}+\frac{h^{2}}{\tilde{\varepsilon}_{13}}\|\mathbf{u}\|_{L^{2}\left(0, \hat{T} ; H^{2}(\Omega)\right)}^{d}\right) \\
& +\frac{\tilde{\varepsilon}_{12}+\tilde{\varepsilon}_{13}}{2} \tau\left|r_{h}^{n}\right|_{H^{1}(\Omega)}^{2} .
\end{aligned}
$$


Finally, by using the obtained bounds and summing over $n$ from 1 to $m \leq N$, we get

$$
\begin{aligned}
\frac{1}{2}\left\|r_{h}^{m}\right\|_{L^{2}(\Omega)}^{2}+\frac{1}{2} \sum_{n=1}^{m}\left\|r_{h}^{n}-r_{h}^{n-1}\right\|_{L^{2}(\Omega)}^{2}+\sum_{n=1}^{m} \alpha \tau\left|r_{h}^{n}\right|_{H^{1}(\Omega)}^{2} \\
\quad \leq \tilde{\xi}_{1}\left(h^{2}+\tau^{2}\right)+\tilde{\xi}_{2} \sum_{n=1}^{m} \tau\left|\mathbf{u}_{h}^{n}-P_{h} \mathbf{u}\left(t_{n}\right)\right|_{H^{1}(\Omega)^{2}}+\tilde{\xi}_{3} \sum_{n=1}^{m} \tau\left|r_{h}^{n}\right|_{H^{1}(\Omega)}^{2}
\end{aligned}
$$

where $\tilde{\xi}_{1}, \tilde{\xi}_{2}$ and $\tilde{\xi}_{3}$ depending of $\tilde{\varepsilon}_{i}, i=1, \ldots, 12$. After a suitable choice of $\tilde{\varepsilon}_{i}$, we conclude the following bound

$$
\begin{gathered}
\frac{1}{2}\left\|r_{h}^{m}\right\|_{L^{2}(\Omega)}^{2}+\frac{1}{2} \sum_{n=1}^{m}\left\|r_{h}^{n}-r_{h}^{n-1}\right\|_{L^{2}(\Omega)}^{2}+\frac{1}{2} \sum_{n=1}^{m} \alpha \tau\left|r_{h}^{n}\right|_{H^{1}(\Omega)}^{2} \\
\leq C_{1}\left(h^{2}+\tau^{2}\right)+C_{2} \sum_{n=1}^{m} \tau\left|\mathbf{u}_{h}^{n}-P_{h} \mathbf{u}\left(t_{n}\right)\right|_{H^{1}(\Omega)^{2}}
\end{gathered}
$$

To establish the a priori error estimates corresponding to the numerical scheme (Eds1), we use Lemma (2.2) which is valid only for $d=2$.

Theorem 4.6. Let $d=2,(\boldsymbol{u}, T, p)$ be the solution of Problem $E$ and $\left(\boldsymbol{u}_{h}^{n}, T_{h}^{n}, p_{h}^{n}\right)$ be the solution of problem (Eds1). If $\boldsymbol{u} \in L^{2}\left(0, \hat{T} ; H^{2}(\Omega)^{2}\right) \cap L^{\infty}\left(0, \hat{T} ; W^{1,4}(\Omega)^{2}\right), \boldsymbol{u}^{\prime} \in L^{2}\left(0, \hat{T} ; H^{2}(\Omega)^{2}\right), T^{\prime} \in L^{2}\left(0, \hat{T} ; H^{1}(\Omega)\right)$ and $p \in L^{2}\left(0, \hat{T} ; H^{1}(\Omega)^{2}\right)$, there exists positive constants $C_{\boldsymbol{u}}^{\prime}, C_{\boldsymbol{u}}^{\prime \prime}$ and $C_{\boldsymbol{u}}^{\prime \prime \prime}$ depending on $\boldsymbol{u}, \boldsymbol{u}^{\prime}, T, T^{\prime}$ and $\alpha$ such that, for any positive real number $\delta_{1}$ and for all $m \leq N$, we have for a time step $\tau$ sufficiently small,

$$
\begin{gathered}
\frac{1}{2}\left\|\mathbf{u}_{h}^{m}-P_{h} \mathbf{u}\left(t_{m}\right)\right\|_{L^{2}(\Omega)^{2}}^{2}+\frac{\tilde{\nu}_{1}}{2} \sum_{n=1}^{m} \tau\left|\mathbf{u}_{h}^{n}-P_{h} \mathbf{u}\left(t_{n}\right)\right|_{H^{1}(\Omega)^{2}}^{2}+\frac{1}{2} \sum_{n=1}^{m}\left\|\mathbf{u}_{h}^{n}-P_{h} \mathbf{u}\left(t_{n}\right)-\left(\mathbf{u}_{h}^{n-1}-P_{h} \mathbf{u}\left(t_{n-1}\right)\right)\right\|_{L^{2}(\Omega)^{2}}^{2} \\
\leq C_{\boldsymbol{u}}^{\prime}\left(h^{2}+\tau^{2}\right)+C_{\boldsymbol{u}}^{\prime \prime} \sum_{n=1}^{m} \frac{\tau \delta_{1}}{2}\left|T_{h}^{n-1}-T\left(t_{n-1}\right)\right|_{H^{1}(\Omega)}^{2}+C_{\boldsymbol{u}}^{\prime \prime} \sum_{n=1}^{m} \frac{\tau}{2 \delta_{1}}\left\|T_{h}^{n}-T\left(t_{n-1}\right)\right\|_{L^{2}(\Omega)}^{2} .
\end{gathered}
$$

Proof. We choose the test function $\mathbf{v}=\mathbf{v}_{h}^{n}=\mathbf{u}_{h}^{n}-P_{h} \mathbf{u}\left(t_{n}\right)$ in the first equation of (E) and then integrate over $\left[t_{n-1}, t_{n}\right]$. We subtract the second equation of (Eds1) multiplied by the time step $\tau$ and insert $\pm P_{h} \mathbf{u}\left(t_{n-1}\right)$ and $\pm P_{h} \mathbf{u}\left(t_{n}\right)$ to obtain :

$$
\begin{aligned}
\frac{1}{2} \| \mathbf{v}_{h}^{n} & \left\|_{L^{2}(\Omega)^{2}}^{2}-\frac{1}{2}\right\| \mathbf{v}_{h}^{n-1}\left\|_{L^{2}(\Omega)^{2}}^{2}+\frac{1}{2}\right\| \mathbf{v}_{h}^{n}-\mathbf{v}_{h}^{n-1} \|_{L^{2}(\Omega)^{2}}^{2}+\tau\left(\nu\left(T_{h}^{n-1}\right) \nabla \mathbf{v}_{h}^{n}, \nabla \mathbf{v}_{h}^{n}\right) \\
= & \int_{t_{n-1}}^{t_{n}}\left(\mathbf{f}_{1}\left(T_{h}^{n-1}\right)-\mathbf{f}_{1}(T(t)), \mathbf{v}_{h}^{n}\right) d t-\int_{t_{n-1}}^{t_{n}}\left(p_{h}^{n}-p(t), \operatorname{div}\left(\mathbf{v}_{h}^{n}\right)\right) d t \\
& -\int_{t_{n-1}}^{t_{n}}\left(\nu\left(T_{h}^{n-1}\right) \nabla P_{h} \mathbf{u}\left(t_{n}\right)-\nu(T(t)) \nabla \mathbf{u}(t), \nabla \mathbf{v}_{h}^{n}\right) d t-\int_{t_{n-1}}^{t_{n}}\left(P_{h} \mathbf{u}^{\prime}(t)-\mathbf{u}^{\prime}(t), \mathbf{v}_{h}^{n}\right) d t \\
& -\int_{t_{n-1}}^{t_{n}}\left(\left(\mathbf{u}_{h}^{n-1} \nabla\right) \cdot \mathbf{u}_{h}^{n}-(\mathbf{u}(t) \nabla) \cdot \mathbf{u}(t), \mathbf{v}_{h}^{n}\right) d t-\frac{1}{2}\left(\tau \operatorname{div}\left(\mathbf{u}_{h}^{n-1}\right) \mathbf{u}_{h}^{n}, \mathbf{v}_{h}^{n}\right) .
\end{aligned}
$$

By using Assumption 2.4, the last term in the left hand side of (4.21) verifies the inequality

$$
\tau\left(\nu\left(T_{h}^{n-1}\right) \nabla \mathbf{v}_{h}^{n}, \nabla \mathbf{v}_{h}^{n}\right) \geq \tau \hat{\nu}_{1}\left|\mathbf{v}_{h}^{n}\right|_{H^{1}(\Omega)^{2}}^{2} .
$$


The first term of the right hand side of (4.21) can be bounded, by using Assumption 2.4 and inserting $\pm \mathbf{f}_{1}\left(T\left(t_{n-1}\right)\right)$, as

$$
\begin{aligned}
\left|\int_{t_{n-1}}^{t_{n}}\left(\mathbf{f}_{1}\left(T_{h}^{n-1}\right)-\mathbf{f}_{1}(T(t)), \mathbf{v}_{h}^{n}\right) d t\right| \leq & c_{\mathbf{f}_{1}}^{*} \int_{t_{n-1}}^{t_{n}}\left\|T_{h}^{n-1}-T\left(t_{n-1}\right)\right\|_{L^{2}(\Omega)}\left\|\mathbf{v}_{h}^{n}\right\|_{L^{2}(\Omega)^{2}} d t \\
& +c_{\mathbf{f}_{1}}^{*} \int_{t_{n-1}}^{t_{n}}\left\|T\left(t_{n-1}\right)-T(t)\right\|_{L^{2}(\Omega)}\left\|\mathbf{v}_{h}^{n}\right\|_{L^{2}(\Omega)^{2}} d t \\
\leq & \frac{\left(S_{4}^{0}\right)^{2}}{2 \varepsilon_{1}}\left(c_{\mathbf{f}_{1}}^{*}\right)^{2} \tau\left\|T_{h}^{n-1}-T\left(t_{n-1}\right)\right\|_{L^{2}(\Omega)}^{2}+\frac{\tau \varepsilon_{1}}{2}\left|\mathbf{v}_{h}^{n}\right|_{H^{1}(\Omega)^{2}}^{2} \\
& +\frac{\left(S_{4}^{0}\right)^{2}}{2 \varepsilon_{2}}\left(c_{\mathbf{f}_{1}}^{*}\right)^{2} \tau^{2}\left\|T^{\prime}\right\|_{L^{2}\left(0, \hat{T} ; L^{2}(\Omega)\right)}^{2}+\frac{\tau \varepsilon_{2}}{2}\left|\mathbf{v}_{h}^{n}\right|_{H^{1}(\Omega)^{2}}^{2} .
\end{aligned}
$$

The second term of the right hand side of (4.21) verifies

$$
\begin{aligned}
\left|\int_{t_{n-1}}^{t_{n}}\left(p_{h}^{n}-p(t), \operatorname{div}\left(\mathbf{v}_{h}^{n}\right)\right) d t\right| & =\left|\int_{t_{n-1}}^{t_{n}}\left(r_{h} p(t)-p(t), \operatorname{div}\left(\mathbf{v}_{h}^{n}\right)\right) d t\right| \\
& \leq \frac{c_{0}^{2} h^{2}}{\varepsilon_{3}}\|p\|_{L^{2}\left(0, \hat{T} ; H^{1}(\Omega)\right)}^{2}+\frac{\varepsilon_{3} \tau}{2}\left|\mathbf{v}_{h}^{n}\right|_{H^{1}(\Omega)^{2}}^{2} .
\end{aligned}
$$

To bound the third term of the right hand side of (4.21), we write

$$
\begin{aligned}
\int_{t_{n-1}}^{t_{n}}\left(\nu\left(T_{h}^{n-1}\right)\right. & \left.\nabla P_{h} \mathbf{u}\left(t_{n}\right)-\nu(T(t)) \nabla \mathbf{u}(t), \nabla \mathbf{v}_{h}^{n}\right) d t=\int_{t_{n-1}}^{t_{n}} \int_{t}^{t_{n}}\left(\nu\left(T_{h}^{n-1}\right) \nabla P_{h} \mathbf{u}^{\prime}(s), \nabla \mathbf{v}_{h}^{n}\right) d s d t \\
& +\int_{t_{n-1}}^{t_{n}}\left(\nu\left(T_{h}^{n-1}\right) \nabla\left(P_{h} \mathbf{u}(t)-\mathbf{u}(t)\right), \nabla \mathbf{v}_{h}^{n}\right) d t+\int_{t_{n-1}}^{t_{n}}\left(\left(\nu\left(T_{h}^{n-1}\right)-\nu\left(T\left(t_{n-1}\right)\right)\right) \nabla \mathbf{u}(t), \nabla \mathbf{v}_{h}^{n}\right) d t \\
& +\int_{t_{n-1}}^{t_{n}}\left(\left(\nu\left(T\left(t_{n-1}\right)\right)-\nu(T(t))\right) \nabla \mathbf{u}(t), \nabla \mathbf{v}_{h}^{n}\right) d t
\end{aligned}
$$

then, we use Assumption 2.4, the properties of $P_{h}$ and the following inequality (based on the relation $(2.2))$

$$
\begin{aligned}
\left\|T_{h}^{n-1}-T\left(t_{n-1}\right)\right\|_{L^{4}(\Omega)}^{2} & \leq 2^{1 / 2}\left\|T_{h}^{n-1}-T\left(t_{n-1}\right)\right\|_{L^{2}(\Omega)}\left|T_{h}^{n-1}-T\left(t_{n-1}\right)\right|_{H^{1}(\Omega)} \\
& \leq 2^{1 / 2}\left(\frac{1}{2 \delta_{1}}\left\|T_{h}^{n-1}-T\left(t_{n-1}\right)\right\|_{L^{2}(\Omega)}^{2}+\frac{1}{2} \delta_{1}\left|T_{h}^{n-1}-T\left(t_{n-1}\right)\right|_{H^{1}(\Omega)}^{2}\right),
\end{aligned}
$$

to get the following bound:

$$
\begin{aligned}
\mid \int_{t_{n-1}}^{t_{n}} & \left.\left(\nu\left(T_{h}^{n-1}\right) \nabla P_{h} \mathbf{u}\left(t_{n}\right)-\nu(T) \nabla \mathbf{u}, \nabla \mathbf{v}_{h}^{n}\right) d t\left|\leq \frac{\left(\hat{\nu_{2}}\right)^{2}\left(c_{P}^{\prime}\right)^{2}}{6 \varepsilon_{4}} \tau^{2}\left\|\mathbf{u}^{\prime}\right\|_{L^{2}\left(0, \hat{T} ; H^{1}(\Omega)^{2}\right)}^{2}+\frac{\tau \varepsilon_{4}}{2}\right| \mathbf{v}_{h}^{n}\right|_{H^{1}(\Omega)^{2}} ^{2} \\
& +\frac{c_{1}}{\varepsilon_{5}} \hat{\nu}_{2}^{2} h^{2}\|\mathbf{u}\|_{L^{2}\left(0, \hat{T} ; H^{2}(\Omega)^{2}\right)}^{2}+\frac{\tau \varepsilon_{5}}{2}\left|\mathbf{v}_{h}^{n}\right|_{H^{1}(\Omega)^{2}}^{2} \\
& +\frac{c_{2}}{\varepsilon_{6}}\left(\nu^{*}\right)^{2} S_{4}^{0}\|\mathbf{u}\|_{L^{\infty}\left(0, \hat{T} ; W^{1,4}(\Omega)^{2}\right)}^{2} \tau\left(\frac{1}{2 \delta_{1}}\left\|T_{h}^{n-1}-T\left(t_{n-1}\right)\right\|_{L^{2}(\Omega)}^{2}+\frac{1}{2} \delta_{1}\left|T_{h}^{n-1}-T\left(t_{n-1}\right)\right|_{H^{1}(\Omega)}^{2}\right) \\
& +\frac{\tau \varepsilon_{6}}{2}\left|\mathbf{v}_{h}^{n}\right|_{H^{1}(\Omega)^{2}}^{2}+\frac{c_{3}}{\varepsilon_{7}}\left(\nu^{*}\right)^{2}\left(S_{4}^{0}\right)^{2}\|\mathbf{u}\|_{L^{\infty}\left(0, \hat{T} ; W^{1,4}(\Omega)^{2}\right)}^{2}\left\|T^{\prime}\right\|_{L^{2}\left(t_{n-1}, t_{n} ; H^{1}(\Omega)\right)}^{2} \tau^{2}+\frac{\tau \varepsilon_{7}}{2}\left|\mathbf{v}_{h}^{n}\right|_{H^{1}(\Omega)^{2}}^{2} .
\end{aligned}
$$

The last two terms of the right hand side of (4.21) can be treated by following the steps used for the study of the corresponding terms if the proof of Theorem 4.17. In fact, by inserting $\pm \tau\left(\left(\mathbf{u}_{h}^{n-1} \nabla\right) P_{h} \mathbf{u}\left(t_{n}\right), \mathbf{v}_{h}^{n}\right)$ and $\pm \frac{\tau}{2}\left(\operatorname{div}\left(\mathbf{u}_{h}^{n-1}\right) P_{h} \mathbf{u}\left(t_{n}\right), \mathbf{v}_{h}^{n}\right)$, the trilinear terms can be written as

$$
a_{n}=\tau\left(\left(\mathbf{u}_{h}^{n-1} \nabla\right) \cdot P_{h} \mathbf{u}\left(t_{n}\right), \mathbf{v}_{h}^{n}\right)+\frac{\tau}{2}\left(\operatorname{div}\left(\mathbf{u}_{h}^{n-1}\right) P_{h} \mathbf{u}\left(t_{n}\right), \mathbf{v}_{h}^{n}\right)-\int_{t_{n-1}}^{t_{n}}\left((\mathbf{u}(t) \nabla) \mathbf{u}(t), \mathbf{v}_{h}^{n}\right) d t
$$


In addition, we have

$$
\begin{aligned}
a_{n}= & \tau\left(\left(\left(\mathbf{u}_{h}^{n-1}-P_{h} \mathbf{u}\left(t_{n-1}\right)\right) \nabla\right) \cdot P_{h} \mathbf{u}\left(t_{n}\right), \mathbf{v}_{h}^{n}\right)+\int_{t_{n-1}}^{t_{n}}\left(\left(P_{h} \mathbf{u}\left(t_{n-1}\right) \nabla\left(P_{h} \mathbf{u}\left(t_{n}\right)-\mathbf{u}(t)\right), \mathbf{v}_{h}^{n}\right)\right) d t \\
& +\int_{t_{n-1}}^{t_{n}}\left(\left(\left(P_{h} \mathbf{u}\left(t_{n-1}\right)-\mathbf{u}(t)\right) \nabla \mathbf{u}(t), \mathbf{v}_{h}^{n}\right) d t+\frac{\tau}{2}\left(\operatorname{div}\left(\mathbf{u}_{h}^{n-1}-P_{h} \mathbf{u}\left(t_{n-1}\right)\right) P_{h} \mathbf{u}\left(t_{n}\right), \mathbf{v}_{h}^{n}\right)\right. \\
& +\frac{1}{2} \int_{t_{n-1}}^{t_{n}}\left(\operatorname{div}\left(P_{h} \mathbf{u}\left(t_{n-1}\right)\right)\left(P_{h} \mathbf{u}\left(t_{n}\right)-\mathbf{u}(t)\right), \mathbf{v}_{h}^{n}\right) d t+\frac{1}{2} \int_{t_{n-1}}^{t_{n}}\left(\operatorname{div}\left(P_{h} \mathbf{u}\left(t_{n-1}\right)-\mathbf{u}(t)\right) \mathbf{u}(t), v_{h}^{n}\right) d t .
\end{aligned}
$$

The second, third, fifth and sixth terms of (4.25) can be treated exactly by following the steps of the corresponding terms in the proof of Theorem 4.17. We have to treat only the sum of the first and fourth terms of (4.25) as following :

$$
\begin{aligned}
c_{n} & =\tau\left(\left(\left(\mathbf{u}_{h}^{n-1}-P_{h} \mathbf{u}\left(t_{n-1}\right)\right) \nabla\right) \cdot P_{h} \mathbf{u}\left(t_{n}\right), \mathbf{v}_{h}^{n}\right)+\frac{\tau}{2}\left(\operatorname{div}\left(\mathbf{u}_{h}^{n-1}-P_{h} \mathbf{u}\left(t_{n-1}\right)\right) P_{h} \mathbf{u}\left(t_{n}\right), \mathbf{v}_{h}^{n}\right) \\
& =\frac{\tau}{2}\left(\left(\left(\mathbf{u}_{h}^{n-1}-P_{h} \mathbf{u}\left(t_{n-1}\right)\right) \nabla\right) \cdot P_{h} \mathbf{u}\left(t_{n}\right), \mathbf{v}_{h}^{n}\right)-\frac{\tau}{2}\left(\left(\left(\mathbf{u}_{h}^{n-1}-P_{h} \mathbf{u}\left(t_{n-1}\right)\right) \nabla\right) \cdot \mathbf{v}_{h}^{n}, P_{h} \mathbf{u}\left(t_{n}\right)\right) .
\end{aligned}
$$

Then we obtain by using Lemma 2.2

$$
\begin{aligned}
\left|c_{n}\right| \leq C_{1} \tau \| \mathbf{u}_{h}^{n-1}-\left.P_{h} \mathbf{u}\left(t_{n-1}\right)\right|_{L^{4}(\Omega)^{2}}\left|\mathbf{v}_{h}^{n}\right|_{H^{1}(\Omega)^{2}}\left|\mathbf{u}\left(t_{n}\right)\right|_{H^{1}(\Omega)^{2}} \\
\leq C_{1}|| \mathbf{u} \|_{L \infty\left(0, \hat{T} ; H^{1}(\Omega)^{2}\right)}\left(\frac{1}{2 \varepsilon_{8}}\left(\frac{\delta_{1}}{2} \tau\left|\mathbf{u}_{h}^{n-1}-P_{h} \mathbf{u}\left(t_{n-1}\right)\right|_{H^{1}(\Omega)^{2}}^{2}+\frac{1}{2 \delta_{1}} \tau\left\|\mathbf{u}_{h}^{n-1}-P_{h} \mathbf{u}\left(t_{n-1}\right)\right\|_{L^{2}(\Omega)^{2}}\right)\right. \\
\left.\frac{1}{2} \varepsilon_{8}\left|\mathbf{v}_{h}^{n}\right|_{H^{1}(\Omega)^{2}}^{2}\right) .
\end{aligned}
$$

Then, by collecting the above bounds and summing over $n$ from 1 to $m \leq N$, we get

$$
\begin{aligned}
\frac{1}{2} \| \mathbf{v}_{h}^{m} & \left\|_{L^{2}(\Omega)^{2}}^{2}+\frac{1}{2} \sum_{n=1}^{m}\right\| \mathbf{v}_{h}^{n}-\mathbf{v}_{h}^{n-1} \|_{L^{2}(\Omega)^{2}}^{2}+\hat{\nu}_{1} \sum_{n=1}^{m} \tau\left|\mathbf{v}_{h}^{n}\right|_{H^{1}(\Omega)^{2}}^{2} \\
\leq & \xi_{1}\left(h^{2}+\tau^{2}\right)+\xi_{2} \sum_{n=1}^{m} \frac{\tau}{2 \delta_{1}}\left\|T_{h}^{n-1}-T\left(t_{n-1}\right)\right\|_{L^{2}(\Omega)}^{2}+\sum_{n=1}^{m} \frac{\tau \delta_{1}}{2}\left|T_{h}^{n}-T\left(t_{n}\right)\right|_{H^{1}(\Omega)}^{2} \\
& +\xi_{3} \sum_{n=1}^{m} \tau\left\|\mathbf{v}_{h}^{n}\right\|_{L^{2}(\Omega)^{2}}^{2}+\xi_{4} \sum_{n=1}^{m} \tau\left\|\mathbf{v}_{h}^{n}\right\|_{H^{1}(\Omega)^{2}}^{2}
\end{aligned}
$$

where $\xi_{1} \xi_{2}, \xi_{3}$ and $\xi_{4}$ depending of $\varepsilon_{i}$. After a suitable choice of $\varepsilon_{i}$, the last term of (4.26) can be absorbed by the term in the left hand side of the inequality. Next, by bounding the term (for $n=m$ ) $\left\|\mathbf{v}_{h}^{m}\right\|_{L^{2}(\Omega)}$ in the right hand side of the last relation as

$$
\left\|\mathbf{v}_{h}^{m}\right\|_{H^{1}(\Omega)^{2}} \leq\left\|\mathbf{v}_{h}^{m}-\mathbf{v}_{h}^{m-1}\right\|_{H^{1}(\Omega)^{2}}+\left\|\mathbf{v}_{h}^{m-1}\right\|_{H^{1}(\Omega)^{2}},
$$

choosing $2 \xi_{3} \tau<1$ and applying the discrete Gronwall Lemma, we get

$$
\begin{aligned}
& \frac{1}{2}\left\|\mathbf{v}_{h}^{m}\right\|_{L^{2}(\Omega)^{2}}^{2}+\frac{1}{2} \sum_{n=1}^{m}\left\|\mathbf{v}_{h}^{n}-\mathbf{v}_{h}^{n-1}\right\|_{L^{2}(\Omega)^{2}}^{2}+\frac{\tilde{\nu}_{1}}{2} \sum_{n=1}^{m} \tau\left|\mathbf{v}_{h}^{n}\right|_{H^{1}(\Omega)^{2}}^{2} \\
& \leq C_{\mathbf{u}}^{\prime}\left(h^{2}+\tau^{2}\right)+\sum_{n=1}^{m} \frac{\tau \delta_{1}}{2}\left|T_{h}^{n-1}-T\left(t_{n-1}\right)\right|_{H^{1}(\Omega)}^{2}+C_{\mathbf{u}}^{\prime \prime} \sum_{n=1}^{m} \frac{\tau}{2 \delta_{1}}\left\|T_{h}^{n-1}-T\left(t_{n-1}\right)\right\|_{L^{2}(\Omega)}^{2} .
\end{aligned}
$$

Theorem 4.7. Under the assumptions of Theorems 4.5 and 4.6, and by choosing $T \in L^{\infty}\left(0, \hat{T}, H^{2}(\Omega)\right)$, there exists constants $k_{0}, C_{u}$ and $C_{T}$, independent of $h$ and $\tau$, such that

$$
\sup _{1 \leq n \leq N}\left\|T_{h}^{n}-T\left(t_{n}\right)\right\|_{L^{2}(\Omega)}^{2}+\alpha \sum_{n=1}^{N} \tau\left|T_{h}^{n}-T\left(t_{n}\right)\right|_{H^{1}(\Omega)}^{2} \leq C_{T}\left(h^{2}+\tau^{2}\right) .
$$

and

$$
\sup _{1 \leq n \leq N}\left\|\boldsymbol{u}_{h}^{n}-\boldsymbol{u}\left(t_{n}\right)\right\|_{L^{2}(\Omega)^{2}}^{2}+\hat{\nu}_{1} \sum_{n=1}^{N} \tau\left|\mathbf{v}_{h}^{n}\right|_{H_{0}^{1}(\Omega)^{2}} \leq C_{\boldsymbol{u}}\left(h^{2}+\tau^{2}\right),
$$

for $\tau<k_{0}$. 
Proof. We insert $\pm R_{h} T\left(t_{n-1}\right)$ in (4.20), use Theorem 4.5, choose $T \in L^{\infty}\left(0, \hat{T}, H^{2}(\Omega)\right)$ and apply the properties of the operator $R_{h}$ to get the following bound

$$
\begin{aligned}
& \frac{1}{2}\left\|r_{h}^{m}\right\|_{L^{2}(\Omega)}^{2}+\frac{1}{2} \sum_{n=1}^{m} \alpha \tau\left|r_{h}^{n}\right|_{H^{1}(\Omega)}^{2}+\frac{1}{2} \sum_{n=1}^{m}\left\|r_{h}^{n}-r_{h}^{n-1}\right\|_{L^{2}(\Omega)}^{2} \\
& \quad \leq C_{1}\left(h^{2}+\tau^{2}\right)+C_{2} \sum_{n=1}^{m} \frac{\tau \delta_{1}}{2}\left|r_{h}^{n-1}\right|_{H^{1}(\Omega)}^{2}+C_{3} \sum_{n=1}^{m} \frac{\tau}{2 \delta_{1}}\left\|r_{h}^{n-1}\right\|_{L^{2}(\Omega)}^{2},
\end{aligned}
$$

where $C_{1}, C_{2}$ and $C_{3}$ are constants independent of $h$ and $\tau$.

We choose $\delta_{1}=\frac{\alpha}{4 C_{2}}$ and we obtain

$$
\frac{1}{2}\left\|r_{h}^{m}\right\|_{L^{2}(\Omega)}^{2}+\frac{1}{4} \sum_{n=1}^{m} \alpha \tau\left|r_{h}^{n}\right|_{H^{1}(\Omega)}^{2} \leq C_{1}\left(h^{2}+\tau^{2}\right)+C_{3} \sum_{n=1}^{m} \tau\left\|r_{h}^{n-1}\right\|_{L^{2}(\Omega)}^{2},
$$

It follows from the discrete Gronwall Lemma the following inequality

$$
\frac{1}{2}\left\|r_{h}^{m}\right\|_{L^{2}(\Omega)}^{2}+\frac{1}{4} \sum_{n=1}^{m} \alpha \tau\left|r_{h}^{n}\right|_{H^{1}(\Omega)}^{2} \leq C_{1}\left(h^{2}+\tau^{2}\right) .
$$

To get the relation (4.28), it suffices to apply

$$
\left|T_{h}^{n}-T\left(t_{n}\right)\right|_{H^{1}(\Omega)} \leq\left|T_{h}^{n}-R_{h} T\left(t_{n}\right)\right|_{H^{1}(\Omega)}+\left|R_{h} T\left(t_{n}\right)-T\left(t_{n}\right)\right|_{H^{1}(\Omega)} .
$$

Finally, the bound (4.29) can be directly deduced from Theorem 4.6.

Remark 4.8. For the numerical scheme (Eds2), we follow the same steps used for (Eds1) with small modifications at the level of the trilinear terms. In fact, (Eds2) doesn't contain the operator div, so the terms $\left(\left(\mathbf{u}_{h}^{n-1} \nabla\right) \cdot \mathbf{v}_{h}^{n}, \mathbf{v}_{h}^{n}\right)$ and $\left(\left(\mathbf{u}_{h}^{n} \nabla\right) \cdot r_{h}^{n}, r_{h}^{n}\right)$ don't vanish. Here we can apply Relations (4.12) and (4.13), and the obtained terms will be absorbed by the corresponding ones in right hand side of the inequality for a space step $h$ smaller than a positive constant $h_{0}$. Thus, we deduce the same a priori error estimates as in Theorem 4.7.

\section{SECOND ORDER DisCRETIZATION}

In this section, we introduce a two order numerical scheme and we establish the corresponding a priori error estimates. In fact, this non-linear scheme needs to be solved at each time step.

For the time discretization, we use the second order BDF method. For the space discretization, we keep the same mesh and notations as in the previous section and we choose the following discrete spaces for the velocity, pressure (Taylor hood finite elements) and temperature:

$$
\begin{gathered}
X_{h}=\left\{\mathbf{v}_{h} \in C^{0}(\bar{\Omega})^{d} ; \forall \kappa \in \mathcal{T}_{h}, \mathbf{v}_{\left.h\right|_{\kappa}} \in \mathbb{P}_{2}(\kappa), \mathbf{v}_{\left.h\right|_{\partial \Omega}}=0\right\}, \\
M_{h}=\left\{q_{h} \in C^{0}(\bar{\Omega}) ; \forall \kappa \in \mathcal{T}_{h}, q_{\left.h\right|_{\kappa}} \in \mathbb{P}_{1}, \int_{\Omega} q_{h} d \mathbf{x}=0\right\},
\end{gathered}
$$

and

$$
Y_{h}=\left\{\theta_{h} \in C^{0}(\bar{\Omega}) ; \forall \kappa \in \mathcal{T}_{h}, \theta_{\left.h\right|_{\kappa}} \in \mathbb{P}_{2}(\kappa), \theta_{\left.h\right|_{\partial \Omega}}=0\right\} .
$$

The previous spaces $X_{h}$ and $M_{h}$ satisfies the inf-sup condition (4.1).

Furthermore, we will introduce new operators, with the same notations of the previous section, $P_{h}, R_{h}$ and $r_{h}$, but adapted to the new discrete spaces.

There exists an approximation operator $P_{h} \in \mathcal{L}\left(H_{0}^{1}(\Omega)^{d} ; X_{h}\right)$ such that (see V. Girault and P.-A. Raviart in $[7])$ :

$$
\forall \mathbf{v} \in H_{0}^{1}(\Omega)^{d}, \quad \forall q_{h} \in M_{h}, \quad \int_{\Omega} q_{h} \operatorname{div}\left(P_{h}(\mathbf{v})-\mathbf{v}\right) d \mathbf{x}=0,
$$

and for $k=0,1$ or 2 ,

$$
\begin{gathered}
\forall \mathbf{v} \in\left[H^{1+k}(\Omega) \cap H_{0}^{1}(\Omega)\right]^{d}, \quad\left\|P_{h}(\mathbf{v})-\mathbf{v}\right\|_{L^{2}(\Omega)^{d}} \leq C_{1} h^{1+k}|v|_{H^{1+k}(\Omega)^{d}} \\
\forall \mathbf{v} \in\left[H^{k+1}(\Omega) \cap H_{0}^{1}(\Omega)\right]^{d}, \quad\left|P_{h}(\mathbf{v})-\mathbf{v}\right|_{H^{1}(\Omega)^{d}} \leq C_{2} h^{k}|\mathbf{v}|_{H^{1+k}(\Omega)^{d}} .
\end{gathered}
$$


As $M_{h}$ preserve the same definition of (4.3), we keep for the pressure the same operator defined in the previous section.

In addition, there exists an operator $R_{h} \in \mathcal{L}\left(H_{0}^{1}(\Omega) ; Y_{h}\right.$ ) (cf. [12]), such that: for any real number $k=0,1$ or 2 ,

$$
\begin{aligned}
\forall r \in H^{k+1}(\Omega) \cap H_{0}^{1}(\Omega), \quad & \left\|R_{h} r-r\right\|_{L^{2}(\Omega)} \leq C_{3} h^{k+1}|r|_{H^{k+1}(\Omega)}, \\
& \left|R_{h} r-r\right|_{H^{1}(\Omega)} \leq c_{4} h^{k}|r|_{H^{k+1}(\Omega)} .
\end{aligned}
$$

We introduce, for $2 \leq n \leq N$, the following stabilized scheme:

for every $n \in\{2, \ldots, N\}$, knowing $\mathbf{u}_{h}^{n-2}$ and $\mathbf{u}_{h}^{n-1} \in X_{h}, T_{h}^{n-2}$ and $T_{h}^{n-1} \in Y_{h}$, we compute $\mathbf{u}_{h}^{n} \in X_{h}, p_{h}^{n} \in$ $M_{h}$ and $T_{h}^{n} \in Y_{h}$ such that for all $\mathbf{v}_{h} \in X_{h}, r_{h} \in Y_{h}$ and $q_{h} \in M_{h}$,

$$
\begin{aligned}
& \left(\frac{3 \mathbf{u}_{h}^{n}-4 \mathbf{u}_{h}^{n-1}+\mathbf{u}_{h}^{n-2}}{2 \tau}, \mathbf{v}_{h}\right)+\left(\nu\left(T_{h}^{n}\right) \nabla \mathbf{u}_{h}^{n}, \nabla \mathbf{v}_{h}\right)-\left(p_{h}^{n}, \operatorname{div} \mathbf{v}_{h}\right)+d_{\mathbf{u}}\left(\mathbf{u}_{h}^{n}, \mathbf{u}_{h}^{n}, \mathbf{v}_{h}\right)=\left(\mathbf{f}^{n}\left(T_{h}^{n}\right), \mathbf{v}_{h}\right), \\
& \left(\frac{3 T_{h}^{n}-4 T_{h}^{n-1}+T_{h}^{n-2}}{2 \tau}, r_{h}\right)+d_{T}\left(\mathbf{u}_{h}^{n}, T_{h}^{n}, r_{h}\right)+\alpha\left(\nabla T_{h}^{n}, \nabla r_{h}\right)=\left(g^{n}, r_{h}\right), \\
& \left(q_{h}, \operatorname{div} \mathbf{u}_{h}^{n}\right)=0,
\end{aligned}
$$

where $\mathbf{u}_{h}^{0}=P_{h} \mathbf{u}(0), T_{h}^{0}=R_{h} T(0), g^{n}=g\left(t_{n}\right)$, and

$$
\mathbf{f}^{n}\left(T_{h}^{n}\right)=\mathbf{f}_{0}^{n}+\mathbf{f}_{1}\left(T_{h}^{n}\right), \quad \text { where } \mathbf{f}_{0}^{n}=\mathbf{f}_{0}\left(t_{n}\right) .
$$

The terms $\mathbf{u}_{h}^{1}$ and $T_{h}^{1}$ are solved by using the following Euler scheme:

$$
\begin{aligned}
& \left(\frac{\mathbf{u}_{h}^{1}-\mathbf{u}_{h}^{0}}{\tau}, \mathbf{v}_{h}\right)+\left(\nu\left(T_{h}^{1}\right) \nabla \mathbf{u}_{h}^{1}, \nabla \mathbf{v}_{h}\right)-\left(p_{h}^{1}, \operatorname{div} \mathbf{v}_{h}\right)+d_{\mathbf{u}}\left(\mathbf{u}_{h}^{1}, \mathbf{u}_{h}^{1}, \mathbf{v}_{h}\right)=\left(\mathbf{f}^{1}\left(T_{h}^{1}\right), \mathbf{v}_{h}\right), \\
& \left(\frac{T_{h}^{1}-T_{h}^{0}}{\tau}, r_{h}\right)+d_{T}\left(\mathbf{u}_{h}^{1}, T_{h}^{1}, r_{h}\right)+\alpha\left(\nabla T_{h}^{1}, \nabla r_{h}\right)=\left(g^{1}, r_{h}\right), \\
& \left(q_{h}, \operatorname{div} \mathbf{u}_{h}^{1}\right)=0 .
\end{aligned}
$$

Theorem 5.1. (Stability of the solution of (5.8))

Let $\left(\mathbf{u}_{h}^{n}, T_{h}^{n}\right)$ in $X_{h} \times M_{h}$ be a solution of Problem (5.8), with the initial data $\left(\mathbf{u}_{h}^{1}, T_{h}^{1}\right)$ and $\left(\mathbf{u}_{h}^{0}, T_{h}^{0}\right)$, we have

$$
\begin{aligned}
\sup _{2 \leq n \leq N} & \left\|\mathbf{u}_{h}^{n}\right\|_{L^{2}(\Omega)^{d}}^{2}+\sup _{2 \leq n \leq N}\left\|2 \mathbf{u}_{h}^{n}-\mathbf{u}_{h}^{n-1}\right\|_{L^{2}(\Omega)^{d}}^{2}+\sum_{n=2}^{N}\left\|\delta^{2} \mathbf{u}_{h}^{n-1}\right\|_{L^{2}(\Omega)^{d}}^{2}+2 \sum_{n=2}^{N} \hat{\nu}_{1} \tau\left|\mathbf{u}_{h}^{n}\right|_{H_{0}^{1}(\Omega)^{d}}^{2} \\
\leq & \bar{C}_{\mathbf{u}^{1}}\left(\|g\|_{L^{\infty}\left(0, \hat{T} ; L^{2}(\Omega)\right)}^{2}+\left\|T_{h}^{1}\right\|_{L^{2}(\Omega)}^{2}+\left\|2 T_{h}^{1}-T_{h}^{0}\right\|_{L^{2}(\Omega)}^{2}+\left\|\mathbf{f}_{0}\right\|_{L^{\infty}\left(0, \hat{T} ; L^{2}(\Omega)^{d}\right)}^{2}\right. \\
& \left.\quad+\left\|\mathbf{u}_{h}^{1}\right\|_{L^{2}(\Omega)^{d}}^{2}+\left\|2 \mathbf{u}_{h}^{1}-\mathbf{u}_{h}^{0}\right\|_{L^{2}(\Omega)^{d}}^{2}\right)
\end{aligned}
$$

and

$$
\begin{aligned}
& \sup _{2 \leq n \leq N}\left\|T_{h}^{n}\right\|_{L^{2}(\Omega)}^{2}+\sup _{2 \leq n \leq N}\left\|2 T_{h}^{n}-T_{h}^{n-1}\right\|_{L^{2}(\Omega)}^{2}+\sum_{n=2}^{N}\left\|\delta^{2} T_{h}^{n-1}\right\|_{L^{2}(\Omega)}^{2}+2 \sum_{n=2}^{N} \alpha \tau\left|T_{h}^{n}\right|_{H_{0}^{1}(\Omega)}^{2} \\
& \quad \leq \bar{C}_{T_{1}}\left(\|g\|_{L^{\infty}\left(0, \hat{T} ; L^{2}(\Omega)\right)}^{2}+\left\|T_{h}^{1}\right\|_{L^{2}(\Omega)}^{2}+\left\|2 T_{h}^{1}-T_{h}^{0}\right\|_{L^{2}(\Omega)}^{2}\right) .
\end{aligned}
$$

where $\bar{C}_{\mathbf{u}^{1}}$ and $\bar{C}_{T_{1}}$ are two positive constants independent of $h$ and $\tau$.

Proof. To prove (5.10) and (5.11), it suffices to take $r_{h}=4 \tau T_{h}^{n}$ and $\mathbf{v}_{h}=4 \tau \mathbf{u}_{h}^{n}$ in System (5.8), using Remark 2.6, Sobolev inequalities and summing over $n$ from 2 to $m \leq N$.

Theorem 5.2. (Existence of the solution of (5.8)) Let $\left(\mathbf{u}_{h}^{1}, T_{h}^{1}\right)$ and $\left(\mathbf{u}_{h}^{0}, T_{h}^{0}\right)$ be the initial data, under Assumption 2.4 Problem (5.8) admits at least one solution. 
Proof. To simplify the proof, we introduce the following constants:

$$
\begin{aligned}
C_{\mathbf{u}_{1}}= & \bar{C}_{\mathbf{u}^{1}}\left(\|g\|_{L^{\infty}\left(0, \hat{T} ; L^{2}(\Omega)\right)}^{2}+\left\|\mathbf{f}_{0}\right\|_{L^{\infty}\left(0, \hat{T} ; L^{2}(\Omega)^{d}\right)}^{2}+\left\|T_{h}^{1}\right\|_{L^{2}(\Omega)}^{2}+\left\|2 T_{h}^{1}-T_{h}^{0}\right\|_{L^{2}(\Omega)}^{2}\right. \\
& \left.+\left\|\mathbf{u}_{h}^{1}\right\|_{L^{2}(\Omega)^{d}}^{2}+\left\|2 \mathbf{u}_{h}^{1}-\mathbf{u}_{h}^{0}\right\|_{L^{2}(\Omega)^{d}}^{2}\right), \\
C_{T_{1}}= & \bar{C}_{T_{1}}\left(\|g\|_{L^{\infty}\left(0, \hat{T} ; L^{2}(\Omega)\right)}^{2}+\left\|T_{h}^{1}\right\|_{L^{2}(\Omega)}^{2}+\left\|2 T_{h}^{1}-T_{h}^{0}\right\|_{L^{2}(\Omega)}^{2}\right) .
\end{aligned}
$$

We proceed by induction on $n \geq 3$. For a given $T_{h}^{n-1}$ and $T_{h}^{n-2} \in Y_{h}$ satisfying (5.11) and (5.10), we introduce the auxiliary problem:

Find $\phi_{T}$ in $Y_{h}$ such that for all $r_{h} \in Y_{h}$,

$$
\left(\phi_{T}\left(T_{h}^{n}\right), r_{h}\right)=\left(\frac{3 T_{h}^{n}-4 T_{h}^{n-1}+T_{h}^{n-2}}{2 \tau}, r_{h}\right)+d_{T}\left(\mathbf{u}_{h}^{n}, T_{h}^{n}, r_{h}\right)+\alpha\left(\nabla T_{h}^{n}, \nabla r_{h}\right)-\left(g^{n}, r_{h}\right) .
$$

On one hand, (5.12) defines a mapping from $Y_{h}$ into $Y_{h}$, and we easily derive its continuity. On the other hand, for $r_{h}=T_{h}^{n}$, we get

$$
\begin{aligned}
\left|\left(\phi\left(T_{h}^{n}\right), T_{h}^{n}\right)\right| & \geq\left|T_{h}^{n}\right|_{H_{0}^{1}(\Omega)}\left(\alpha\left|T_{h}^{n}\right|_{H_{0}^{1}(\Omega)}-\left(\frac{S_{2}^{0}}{2 \tau}\left\|4 T_{h}^{n-1}-T_{h}^{n-2}\right\|_{L^{2}(\Omega)}+S_{2}^{0}\left\|g^{n}\right\|_{L^{2}(\Omega)}\right)\right) \\
& \geq\left|T_{h}^{n}\right|_{H_{0}^{1}(\Omega)}\left(\alpha\left|T_{h}^{n}\right|_{H_{0}^{1}(\Omega)}-\left(\frac{5 S_{2}^{0}}{2 \tau} C_{T_{1}}+S_{2}^{0}\|g\|_{L^{\infty}\left(0, \hat{T} ; L^{2}(\Omega)\right.}\right)\right) .
\end{aligned}
$$

In other words, $\left|\left(\phi\left(T_{h}^{n}\right), T_{h}^{n}\right)\right| \geq 0$ for all $T_{h}^{n}$ in $Y_{h}$ such that

$$
\left|T_{h}^{n}\right|_{H_{0}^{1}(\Omega)}=\frac{1}{\alpha}\left(\frac{5 S_{2}^{0}}{2 \tau} C_{T_{1}}+S_{2}^{0}\|g\|_{L^{\infty}\left(0, \hat{T} ; L^{2}(\Omega)\right.}\right) .
$$

Therefore Brouwer's Fixed-Point Theorem, see for example [7], implies immediately the existence of $T_{h}^{n}$. For the proof of the existence of the velocity, we follow the same method applied to the function $\phi_{\mathbf{u}}$ defined in $X_{h}$, for each $\mathbf{u}_{h}^{n-1}$ and $\mathbf{u}_{h}^{n-2} \in X_{h}$ :

$$
\left(\phi_{\mathbf{u}}\left(\mathbf{u}_{h}^{n}\right), \mathbf{v}_{h}\right)=\left(\frac{3 \mathbf{u}_{h}^{n}-4 \mathbf{u}_{h}^{n-1}+\mathbf{u}_{h}^{n-2}}{2 \tau}, \mathbf{v}_{h}\right)+d_{\mathbf{u}}\left(\mathbf{u}_{h}^{n}, \mathbf{u}_{h}^{n}, \mathbf{v}_{h}\right)+\left(\nu\left(T_{h}^{n}\right) \nabla \mathbf{u}_{h}^{n}, \nabla \mathbf{v}_{h}\right)-\left(\mathbf{f}^{n}\left(T_{h}^{n}\right), \mathbf{v}_{h}\right)
$$

Where

$$
\left|\left(\phi\left(\mathbf{u}_{h}^{n}\right), \mathbf{u}_{h}^{n}\right)\right| \geq\left|\mathbf{u}_{h}^{n}\right|_{H_{0}^{1}(\Omega)^{d}}\left(\hat{\nu}_{1}\left|\mathbf{u}_{h}^{n}\right|_{H_{0}^{1}(\Omega)^{d}}-\left(\frac{5 S_{2}^{0}}{2 \tau} C_{\mathbf{u}_{1}}+c_{\mathbf{f}_{1}} S_{2}^{0} C_{T_{1}}+S_{2}^{0}\left\|\mathbf{f}_{0}\right\|_{L^{\infty}\left(0, \hat{T} ; L^{2}(\Omega)^{d}\right.}\right)\right)
$$

for all $\mathbf{u}_{h}^{n}$ in $X_{h}$ such that

$$
\left|\mathbf{u}_{h}^{n}\right|_{H_{0}^{1}(\Omega)^{d}}=\mu_{\mathbf{u}}=\frac{5 S_{2}^{0}}{2 \tau} C_{\mathbf{u}_{1}}+c_{\mathbf{f}_{1}} S_{2}^{0} C_{T_{1}}+S_{2}^{0}\left\|\mathbf{f}_{0}\right\|_{L^{\infty}\left(0, \hat{T} ; L^{2}(\Omega)^{d}\right)} .
$$

We conclude the existence of the solution of Problem (5.8).

Theorem 5.3. (Uniqueness of the solution of (5.8))

Assume that $\nu$ is lipschitz-continuous, with Lipschitz constant $\nu^{*}$. Under Assumption 2.4, Problem (5.8) has a unique solution $\left(\mathbf{u}_{h}^{n}, p_{h}^{n}, T_{h}^{n}\right)$ in $X_{h} \times M_{h} \times Y_{h}$ for a time step $\tau$ sufficiently small.

Proof. Let $\left(\mathbf{u}_{h}^{(1) n}, p_{h}^{(1) n}, T_{h}^{(1) n}\right)$ and $\left(\mathbf{u}_{h}^{(2) n}, p_{h}^{(2) n}, T_{h}^{(2) n}\right)$ be two solutions of Problem (5.8) and let

$$
\left(\mathbf{w}_{h}^{n}, s_{h}^{n}, z_{h}^{n}\right)=\left(\mathbf{u}_{h}^{(1) n}-\mathbf{u}_{h}^{(2) n}, p_{h}^{(1) n}-p_{h}^{(2) n}, T_{h}^{(1) n}-T_{h}^{(2) n}\right) .
$$

The first two equations of System (5.8), mutiplied by $4 \tau$, allows us to get by using Remark 2.6, Theorem 5.8 and the equivalence of norms in finite dimension spaces, the following relation

$$
\begin{gathered}
\left\|z_{h}^{n}\right\|_{L^{2}(\Omega)}^{2}+\left\|2 z_{h}^{n}-z_{h}^{n-1}\right\|_{L^{2}(\Omega)}^{2}+\left\|\delta^{2} z_{h}^{n-1}\right\|_{L^{2}(\Omega)}^{2}-\left\|z_{h}^{n-1}\right\|_{L^{2}(\Omega)}^{2}-\left\|2 z_{h}^{n-1}-z_{h}^{n-2}\right\|_{L^{2}(\Omega)}^{2} \\
+4 \tau \alpha\left|z_{h}^{n}\right|_{H^{1}(\Omega)}^{2}=-d_{T}\left(\mathbf{w}_{h}^{n}, T_{h}^{(1) n}, z_{h}^{n}\right)+d_{T}\left(\mathbf{u}_{h}^{(2) n}, z_{h}^{n}, z_{h}^{n}\right) \\
\leq c_{1} \tau\left(\left\|\mathbf{w}_{h}^{n}\right\|_{L^{2}(\Omega)^{d}}^{2}+\|\left. z_{h}^{n}\right|_{L^{2}(\Omega)} ^{2}\right)
\end{gathered}
$$


and

$$
\begin{aligned}
& \left\|\mathbf{w}_{h}^{n}\right\|_{L^{2}(\Omega)^{d}}^{2}+\left\|2 \mathbf{w}_{h}^{n}-\mathbf{w}_{h}^{n-1}\right\|_{L^{2}(\Omega)^{d}}^{2}+\left\|\delta^{2} \mathbf{w}_{h}^{n-1}\right\|_{L^{2}(\Omega)^{d}}^{2}-\left\|\mathbf{w}_{h}^{n-1}\right\|_{L^{2}(\Omega)^{d}}^{2} \\
& -\left\|2 \mathbf{w}_{h}^{n-1}-\mathbf{w}_{h}^{n-2}\right\|_{L^{2}(\Omega)^{d}}^{2}+4 \tau \alpha\left|\mathbf{w}_{h}^{n}\right|_{H^{1}(\Omega)^{d}}^{2} \\
& \quad=4 \tau\left(\left(\nu\left(T_{h}^{(1) n}\right)-\nu\left(T_{h}^{(2) n}\right) \nabla \mathbf{u}_{h}^{(1) n}, \nabla \mathbf{w}_{h}^{n}\right)-4 \tau d_{\mathbf{u}}\left(\mathbf{w}_{h}^{n}, \mathbf{u}_{h}^{n}, \mathbf{w}_{h}^{n}\right)+4 \tau\left(\mathbf{f}_{1}\left(T_{h}^{(1) n}\right)-\mathbf{f}_{1}\left(T_{h}^{(2) n}\right), \nabla \mathbf{w}_{h}^{n}\right)\right. \\
& \quad \leq c_{2} \tau\left(\left\|\mathbf{w}_{h}^{n}\right\|_{L^{2}(\Omega)^{d}}^{2}+\left\|z_{h}^{n}\right\|_{L^{2}(\Omega)}^{2}\right),
\end{aligned}
$$

where $c_{1}$ and $c_{2}$ depend of $h$ and are independent of $\tau$.

By summing the last two bounds over $n$ from 2 to $m \leq N$ and remarking that $z_{h}^{0}=z_{h}^{1}=0$ and $\mathbf{w}_{h}^{0}=$ $\mathbf{w}_{h}^{1}=\mathbf{0}$, we get

$$
\begin{aligned}
& \left\|z_{h}^{m}\right\|_{L^{2}(\Omega)}^{2}+\left\|2 z_{h}^{m}-z_{h}^{m-1}\right\|_{L^{2}(\Omega)}^{2}+\sum_{n=1}^{m}\left\|\delta^{2} z_{h}^{n-1}\right\|_{L^{2}(\Omega)}^{2}+4 \alpha \sum_{n=1}^{m} \tau\left|z_{h}^{n}\right|_{H^{1}(\Omega)}^{2}+\left\|\mathbf{w}_{h}^{m}\right\|_{L^{2}(\Omega)^{d}}^{2} \\
& +\left\|2 \mathbf{w}_{h}^{m}-\mathbf{w}_{h}^{m-1}\right\|_{L^{2}(\Omega)^{d}}^{2}+\sum_{n=1}^{m}\left\|\delta^{2} \mathbf{w}_{h}^{n-1}\right\|_{L^{2}(\Omega)^{d}}^{2}+4 \alpha \sum_{n=1}^{m} \tau\left|\mathbf{w}_{h}^{n}\right|_{H^{1}(\Omega)^{d}}^{2} \\
& \leq c_{3} \sum_{n=1}^{m} \tau\left(\left\|\mathbf{w}_{h}^{n}\right\|_{L^{2}(\Omega)^{d}}^{2}+\left\|z_{h}^{n}\right\|_{L^{2}(\Omega)}^{2}\right) .
\end{aligned}
$$

where $c_{3}$ depends of $h$ and is independent of $\tau$.

To apply the Gronwall lemma, we write the last term (for $n=m$ ) of the right hand side of the (5.16) as $\mathbf{w}_{h}^{m}=\delta^{2} \mathbf{w}_{h}^{m-1}+2 \mathbf{w}_{h}^{m-1}-\mathbf{w}_{h}^{m-2}$ and $z_{h}^{n}=\delta^{2} z_{h}^{m-1}+2 z_{h}^{m-1}-z_{h}^{m-2}$ and we get

$$
\left\|\mathbf{w}_{h}^{m}\right\|_{L^{2}(\Omega)^{d}}^{2} \leq 2\left(\left\|\delta^{2} \mathbf{w}_{h}^{m-1}\right\|_{L^{2}(\Omega)^{d}}^{2}+\left\|2 \mathbf{w}_{h}^{m-1}-\mathbf{w}_{h}^{m-2}\right\|_{L^{2}(\Omega)^{d}}^{2}\right)
$$

and

$$
\left\|z_{h}^{n}\right\|_{L^{2}(\Omega)}^{2} \leq 2\left(\left\|\delta^{2} z_{h}^{m-1}\right\|_{L^{2}(\Omega)}^{2}+\left\|2 z_{h}^{m-1}-z_{h}^{m-2}\right\|_{L^{2}(\Omega)}^{2}\right) .
$$

For a time step $\tau$ syfficiently small, the term $c_{3} \tau\left(\left\|z_{h}^{m}\right\|_{L^{2}(\Omega)}^{2}+\left\|\mathbf{w}_{h}^{m}\right\|_{L^{2}(\Omega)^{d}}^{2}\right)$ can be absorbed by the term in the left hand side of the inequality. Applying Gronwall's lemma, we obtain $\mathbf{w}_{h}^{n}=0$ and $z_{h}^{n}=0$. The inf-sup condition implies $s_{h}^{n}=0$ and then we get the uniqueness of the solution.

In the next proposition, we will establish the error estimate for the solution computed by one iteration of Euler's scheme $\left(\mathbf{u}_{h}^{1}-\mathbf{u}(\tau), T_{h}^{1}-T(\tau)\right)$ :

Theorem 5.4. If $T \in L^{\infty}\left(0, \hat{T} ; H^{3}(\Omega)\right), T^{\prime} \in L^{\infty}\left(0, \hat{T} ; H^{2}(\Omega)\right), T^{\prime \prime} \in L^{\infty}\left(0, \hat{T} ; L^{2}(\Omega)\right)$ and $\mathbf{u} \in$ $L^{\infty}\left(0, \hat{T} ; H^{3}(\Omega)\right)$, the error of the solution computed by one iteration of Euler's scheme satisfies the following estimation, for a time step sufficiently small,

$$
\frac{1}{2}\left\|r_{h}^{1}\right\|_{L^{2}(\Omega)}^{2}+\frac{1}{2} \alpha \tau\left|r_{h}^{1}\right|_{H_{0}^{1}(\Omega)}^{2} \leq \hat{C}_{T}^{\prime}\left(h^{4}+\tau^{4}\right)+\hat{C}_{T}^{\prime \prime} \tau\left|\mathbf{v}_{h}^{1}\right|_{H_{0}^{1}(\Omega)^{2}}^{2}
$$

where $r_{h}^{1}=T_{h}^{1}-R_{h} T(\tau), \mathbf{v}_{h}=\mathbf{u}_{h}^{1}-P_{h} \mathbf{u}(\tau)$ and $\hat{C}_{T}^{\prime}$ is independent of $h$ and $\tau$.

Proof. Due to the regularity assumption of $T$, and forall $x$, there exists $\theta \in] 0,1[$ that depends on $x$ such that

$$
T(0)=T(\tau)-\tau T^{\prime}(\tau)+\frac{1}{2} \tau^{2} T^{\prime \prime}(\tau \theta),
$$

and $T_{h}^{1}$ satisfies, by denoting $r_{h}^{1}=T_{h}^{1}-R_{h} T(\tau)$, the error equation

$$
\begin{aligned}
& \left\|r_{h}^{1}\right\|_{L^{2}(\Omega)}^{2}+\alpha \tau\left|r_{h}^{1}\right|_{H^{1}(\Omega)}^{2}=-\left(\left(R_{h} T^{1}-T(\tau)\right)-\left(T_{h}^{0}-T(0)\right), r_{h}^{1}\right) \\
& \quad+\alpha \tau\left(\nabla\left(R_{h} T(\tau)-T(\tau)\right), \nabla r_{h}^{1}\right)-\left(\tau d_{T}\left(\mathbf{u}_{h}^{1}, T_{h}^{1}, r_{h}^{1}\right)-\tau d_{T}\left(\mathbf{u}(\tau), T(\tau), r_{h}^{1}\right)\right)-\frac{\tau^{2}}{2}\left(T^{\prime \prime}(\tau \theta), r_{h}\right) .
\end{aligned}
$$

Then (5.17) follows readily by applying the error approximation of $R_{h}$. 
Theorem 5.5. Let $d=2$ and Assumption (2.4) holds. If $\mathbf{u} \in L^{\infty}\left(0, \hat{T} ; H^{3}(\Omega)^{2}\right)$, $\mathbf{u}^{\prime} \in L^{\infty}\left(0, \hat{T} ; L^{2}(\Omega)^{2}\right)$ and $p \in L^{\infty}\left(0, \hat{T} ; H^{2}(\Omega)^{2}\right)$, then for a time step $\tau$ sufficiently small and for any positive real number $\delta_{1}$, there exists $\bar{C}_{\mathbf{u}}^{\prime}$ and $\hat{C}_{\mathbf{u}}^{\prime \prime}$ in $\mathbb{R}_{*}^{+}$independent of $\delta_{1}$, and $\bar{C}_{\mathbf{u}}^{\prime \prime}$ depending of $\delta_{1}$ such that

$$
\frac{1}{2}\left\|\mathbf{v}_{h}^{1}\right\|_{L^{2}(\Omega)^{2}}^{2}+\frac{\tau \hat{\nu}_{1}}{2}\left|\mathbf{v}_{h}^{1}\right|_{H_{0}^{1}(\Omega)^{2}}^{2} \leq \hat{C}_{\mathbf{u}}^{\prime}\left(h^{4}+\tau^{4}\right)+\bar{C}_{\mathbf{u}}^{\prime \prime} \tau\left\|T_{h}^{1}-T(\tau)\right\|_{L^{2}(\Omega)}^{2}+\hat{C}_{\mathbf{u}}^{\prime \prime} \delta_{1} \tau\left|T_{h}^{1}-T(\tau)\right|_{H_{0}^{1}(\Omega)}^{2} .
$$

Proof. The proof follows the same steps of Theorem 5.4.

Due to the regularity assumption of $\mathbf{u}$, and forall $x$, there exists $\theta \in] 0,1[$ that depends on $x$ such that

$$
\mathbf{u}(0)=\mathbf{u}(\tau)-\tau \mathbf{u}^{\prime}(\tau)+\frac{1}{2} \tau^{2} \mathbf{u}^{\prime \prime}(\tau \theta),
$$

and $\mathbf{u}_{h}^{1}$ satisfies, by denoting $\mathbf{v}_{h}^{1}=\mathbf{v}_{h}^{1}-P_{h} \mathbf{u}(\tau)$, the error equation

$$
\begin{aligned}
\| \mathbf{v}_{h}^{1} & \|_{L^{2}(\Omega)}^{2}+\hat{\nu}_{1} \tau\left|\mathbf{v}_{h}^{1}\right|_{H^{1}(\Omega)}^{2} \leq-\left(\left(P_{h} \mathbf{u}(\tau)-\mathbf{u}(\tau)\right)-\left(\mathbf{u}_{h}^{0}-\mathbf{u}(0)\right), \mathbf{v}_{h}^{1}\right)+\tau\left(r_{h} p(\tau)-p(\tau), \operatorname{div} \mathbf{v}_{h}^{1}\right) \\
& +\tau\left(\nu\left(T_{h}^{1}\right) \nabla P_{h} \mathbf{u}(\tau), \mathbf{v}_{h}^{1}\right)-\left(\nu(T(\tau)) \nabla \mathbf{u}(\tau), \nabla \mathbf{v}_{h}^{1}\right)-\left(\tau d_{\mathbf{u}}\left(\mathbf{u}_{h}^{1}, \mathbf{u}_{h}^{1}, \mathbf{v}_{h}^{1}\right)-\tau d_{\mathbf{u}}\left(\mathbf{u}(\tau), \mathbf{u}(\tau), \mathbf{v}_{h}^{1}\right)\right) \\
& -\frac{\tau^{2}}{2}\left(\mathbf{u}^{\prime \prime}(\tau \theta), \mathbf{v}_{h}^{1}\right)+\tau\left(\mathbf{f}_{1}\left(T_{h}^{1}\right)-\mathbf{f}_{1}(T(\tau)), \mathbf{v}_{h}^{1}\right) .
\end{aligned}
$$

Then (5.19) follows readily by applying the error approximation of $P_{h}$ and $r_{h}$.

Theorem 5.6. Under the assumptions of theorems 5.4 and 5.5 , there exists positive constants $k_{0}, \hat{C}_{\mathbf{u}}$ and $\hat{C}_{T}$, independent of $h$ and $\tau$, such that

$$
\frac{1}{4}\left\|r_{h}^{1}\right\|_{L^{2}(\Omega)}^{2}+\frac{1}{4}\left|r_{h}^{1}\right|_{H_{0}^{1}(\Omega)}^{2} \leq \hat{C}_{T}\left(h^{4}+\tau^{4}\right)
$$

and

$$
\frac{1}{4}\left\|\mathbf{v}_{h}^{1}\right\|_{L^{2}(\Omega)^{d}}^{2}+\frac{1}{4}\left|\mathbf{v}_{h}^{1}\right|_{H_{0}^{1}(\Omega)^{d}}^{2} \leq \hat{C}_{\mathbf{u}}\left(h^{4}+\tau^{4}\right) .
$$

Proof. Theorems 5.4 and 5.5 gives

$$
\frac{1}{2}\left\|r_{h}^{1}\right\|_{L^{2}(\Omega)}^{2}+\frac{1}{2} \alpha \tau\left|r_{h}^{1}\right|_{H_{0}^{1}(\Omega)}^{2} \leq \hat{C}_{T}^{\prime}\left(h^{4}+\tau^{4}\right)+\hat{C}_{T}^{\prime \prime} \tau\left|\mathbf{v}_{h}^{1}\right|_{H_{0}^{1}(\Omega)^{d}}^{2}
$$

and

$$
\frac{\tau \hat{\nu}_{1}}{2}\left|\mathbf{v}_{h}^{1}\right|_{H_{0}^{1}(\Omega)^{d}}^{2} \leq C_{\mathbf{u}}^{\prime}\left(h^{4}+\tau^{4}\right)+\bar{C}_{\mathbf{u}}^{\prime \prime} \tau\left\|T_{h}^{1}-T(\tau)\right\|_{L^{2}(\Omega)}^{2}+C_{\mathbf{u}}^{\prime \prime} \delta_{1} \tau\left|T_{h}^{1}-T(\tau)\right|_{H_{0}^{1}(\Omega)}^{2} .
$$

By Inserting $R_{h} T_{h}^{1}$ in (5.22), using the properties of $R_{h}$, setting $\delta_{1}=\frac{\alpha \hat{\nu}_{1}}{8 \hat{C}_{T}^{\prime \prime} \hat{C}_{\mathbf{u}}^{\prime \prime}}$ and using (5.21), we get

$$
\frac{1}{2}\left\|r_{h}^{1}\right\|_{L^{2}(\Omega)}^{2}+\frac{\alpha \tau}{4}\left|r_{h}^{1}\right|_{H_{0}^{1}(\Omega)}^{2} \leq \hat{C}^{\prime}\left(h^{4}+\tau^{4}\right)+\hat{C}^{\prime \prime} \tau\left\|r_{h}^{1}\right\|_{L^{2}(\Omega)}^{2} .
$$

We choose $\hat{C}^{\prime \prime} \tau \leq \frac{1}{4}$ to conclude

$$
\left\|T_{h}^{1}-T(\tau)\right\|_{L^{2}(\Omega)}^{2}+\tau\left|T_{h}^{1}-T(\tau)\right|_{H_{0}^{1}(\Omega)}^{2} \leq \hat{C}_{T}\left(h^{4}+\tau^{4}\right) .
$$

The error of the velocity comes from Theorem 5.5 and (5.24).

We can now state the first result of error estimate. For the simplicity, from now on we denote $T^{n}=T\left(t_{n}\right)$ and $\mathbf{u}^{n}=\mathbf{u}\left(t_{n}\right)$.

Theorem 5.7. Let $d=2,(\boldsymbol{u}, T, p)$ be the solution of Problem (E) and $\left(\boldsymbol{u}_{h}^{n}, T_{h}^{n}, p_{h}^{n}\right)$ the solution of problem (5.8). If $\boldsymbol{u} \in L^{\infty}\left(0, T ; H^{3}(\Omega)^{2}\right), T \in L^{\infty}\left(0, T ; H^{3}(\Omega)\right), T^{\prime} \in L^{\infty}\left(0, T ; H^{2}(\Omega)\right)$ and $T^{(3)} \in$ 
$L^{2}\left(0, T ; L^{2}(\Omega)\right)$, there exists positive constants $\hat{C}_{T}^{\prime}$ and $\hat{C}_{T}^{\prime \prime}$ independent of $h$ and $\tau$ such that, for all $m \leq N$

$$
\begin{aligned}
& \left\|T_{h}^{m}-R_{h} T\left(t_{m}\right)\right\|_{L^{2}(\Omega)}^{2}+\left\|2\left(T_{h}^{m}-R_{h} T\left(t_{m}\right)\right)-\left(T_{h}^{m-1}-R_{h} T\left(t_{m-1}\right)\right)\right\|_{L^{2}(\Omega)}^{2} \\
& +\sum_{n=2}^{m}\left\|\delta^{2}\left(T_{h}^{n-1}-R_{h} T\left(t_{n-1}\right)\right)\right\|_{L^{2}(\Omega)}^{2}+\sum_{n=2}^{m} \tau \alpha\left|T_{h}^{n}-R_{h} T^{n}\right|_{H^{1}(\Omega)}^{2} \\
& \quad \leq \hat{C}_{T}^{\prime}\left(\tau^{4}+h^{4}\right)+\hat{C}_{T}^{\prime \prime} \sum_{n=2}^{m} \tau\left|\mathbf{u}_{h}^{n}-P_{h} \mathbf{u}^{n}\right|_{H_{0}^{1}(\Omega)^{2}}^{2}
\end{aligned}
$$

Proof. We set $r_{h}=r_{h}^{n}=T_{h}^{n}-R_{h} T\left(t_{n}\right)$ and $\varphi_{h}^{n}=R_{h} T\left(t_{n}\right)-T\left(t_{n}\right)$. We start by adding and substracting $\pm \frac{1}{\tau}\left(3 T\left(t_{n}\right)-4 T\left(t_{n-1}\right)+T\left(t_{n-2}\right), r_{h}\right)$ and $\pm \frac{1}{\tau}\left(3 R_{h} T\left(t_{n}\right)-4 R_{h} T\left(t_{n-1}\right)+R_{h} T\left(t_{n-2}\right), r_{h}\right)$ in the second equation of (5.8), we obtain

$$
\begin{aligned}
& 2\left(\frac{3 r_{h}^{n}-4 r_{h}^{n-1}+r_{h}^{n-2}}{2 \tau}, r_{h}\right)+2\left(\frac{3 \varphi_{h}^{n}-4 \varphi_{h}^{n-1}+\varphi_{h}^{n-2}}{2 \tau}, r_{h}\right)+4 \tau d_{T}\left(\mathbf{u}_{h}^{n}, T_{h}^{n}, r_{h}^{n}\right) \\
& +4 \tau \alpha\left(\nabla T_{h}^{n}, \nabla r_{h}\right)+2\left(\frac{3 T\left(t_{n}\right)-4 T\left(t_{n-1}\right)+T\left(t_{n-2}\right)}{2 \tau}, r_{h}\right)=4 \tau\left(g^{n}, r_{h}\right) .
\end{aligned}
$$

In the other hand, we consider the second equation of (E) taken at $t=t_{n}$. We obtain by inserting $\pm 2\left(3 T\left(t_{n}\right)-4 T\left(t_{n-1}\right)+T\left(t_{n-2}\right), r_{h}^{n}\right)$ and choosing $r=r_{h}^{n}$

$$
2\left(3 T\left(t_{n}\right)-4 T\left(t_{n-1}\right)+T\left(t_{n-2}\right), r_{h}^{n}\right)+4 \tau c_{T}\left(\mathbf{u}_{h}^{n}, T\left(t_{n}\right), r_{h}^{n}\right) \quad+4 \tau \alpha\left(\nabla T_{h}^{n}, \nabla r_{h}^{n}\right)=4 \tau\left(g^{n}, r_{h}^{n}\right)+R_{1},
$$

where

Due to Taylor's formula with integral remainder, we have

$$
R_{1}=\left(T^{\prime}(t)-\frac{3 T(t)-4 T(t-\tau)+T(t-2 \tau)}{2 \tau}, r_{h}\right) .
$$

$$
\left|T^{\prime}(t)-\frac{3 T(t)-4 T(t-\tau)+T(t-2 \tau)}{2 \tau}\right| \leq \frac{\tau^{\frac{3}{2}}}{2 \sqrt{3}}\left\|T^{(3)}(x)\right\|_{L^{2}(t-2 \tau, t)} .
$$

Then, we substract (5.26) and (5.27) and insert $\pm 4 \tau \alpha\left(\nabla R_{h} T^{n}, \nabla r_{h}\right)$. We get,

$$
\begin{aligned}
& \left\|r_{h}^{n}\right\|_{L^{2}(\Omega)}^{2}+\left\|2 r_{h}^{n}-r_{h}^{n-1}\right\|_{L^{2}(\Omega)}^{2}+\left\|\delta r_{h}^{n-1}\right\|_{L^{2}(\Omega)}^{2} \\
& -\left\|r_{h}^{n-1}\right\|_{L^{2}(\Omega)}^{2}-\left\|2 r_{h}^{n-1}-r_{h}^{n-2}\right\|_{L^{2}(\Omega)}^{2}+4 \tau \alpha\left|r_{h}^{n}\right|_{H^{1}(\Omega)}^{2} \\
& \quad \leq\left|R_{1}\right|+\left|2\left(3 \varphi_{h}^{n}-4 \varphi_{h}^{n-1}+\varphi_{h}^{n-2}, r_{h}^{n}\right)\right|+\tau \alpha\left|\left(\nabla \varphi_{h}^{n}, \nabla r_{h}^{n}\right)\right| \\
& \quad+\left|d_{T}\left(\mathbf{u}_{h}^{n}, T_{h}^{n}, r_{h}^{n}\right)-d_{T}\left(\mathbf{u}\left(t_{n}\right), T\left(t_{n}\right), r_{h}^{n}\right)\right| .
\end{aligned}
$$

We denote by $A_{1}, A_{2}$ and $A_{3}$ the second, third and fourth terms of the right hand side of (5.29). We have by using the Cauchy-Schwarz inequality and (2.4)

$$
\left|R_{1}\right| \leq \frac{\tau^{4}}{24 \varepsilon_{1}}\left\|T^{(3)}\right\|_{L^{2}\left(0, \hat{T} ; L^{2}(\Omega)\right)}^{2}+\frac{\tau \varepsilon_{1} S_{2}^{2}}{2}\left|r_{h}^{n}\right|_{H^{1}(\Omega)}^{2} .
$$

In other hand, due to the Taylor's formula, we have

$$
2\left(3 \varphi_{h}^{n}-4 \varphi_{h}^{n-1}+\varphi_{h}^{n-2}, r_{h}^{n}\right)=\tau\left(R_{h} T^{\prime}\left(t_{n}\right)-T^{\prime}\left(t_{n}\right), r_{h}^{n}\right)+\tau\left(R_{2}, r_{h}^{n}\right)
$$

and

$$
\left|R_{2}\right| \leq \frac{\tau^{\frac{3}{2}}}{2 \sqrt{3}}\left\|R_{h} T^{(3)}-T^{(3)}\right\|_{L^{2}\left(t_{n-2}, t_{n}\right)} .
$$

Hence by using Cauchy Schwarz inequality, (5.7) and the stability of $R_{h}$ in $L^{2}(\Omega)$ (see [12]), we have

$$
\begin{aligned}
\left|A_{1}\right| \leq & \frac{c_{1} h^{4} \tau}{2 \varepsilon_{2}}\left|T^{\prime}\right|_{L^{\infty}\left(0, \hat{T} ; H^{2}(\Omega)\right)}^{2}+\frac{\varepsilon_{2}\left(S_{2}^{0}\right)^{2} \tau}{2}\left|r_{h}^{n}\right|_{H^{1}(\Omega)}^{2} \\
& +\frac{\tau^{4} c_{2}}{6 \varepsilon_{3}}\left\|T^{(3)}\right\|_{L^{2}\left(0, \hat{T} ; L^{2}(\Omega)\right)}+\frac{\tau \varepsilon_{3}\left(S_{2}^{0}\right)^{2}}{2}\left|r_{h}^{n}\right|_{H^{1}(\Omega)}^{2} .
\end{aligned}
$$

The terme $A_{2}$ is bounded as follow

$$
\left|A_{2}\right| \leq \frac{\alpha^{2} \tau h^{4} c_{3}}{2 \varepsilon_{4}}\|\mathbf{u}\|_{L^{\infty}\left(0, \hat{T} ; H^{3}(\Omega)^{2}\right)}^{2}+\frac{\tau \varepsilon_{4}}{2}\left|r_{h}^{n}\right|_{H^{1}(\Omega)}^{2} .
$$


Finally, $\left|A_{3}\right|$ is treated as follow

$$
\left|A_{3}\right| \leq\left|A_{3,1}\right|+\left|A_{3,2}\right|+\left|A_{3,3}\right|
$$

where

$$
\begin{gathered}
A_{3,1}=4 \tau\left(\mathbf{v}_{h}^{n} \nabla R_{h} T\left(t_{n}\right), r_{h}^{n}\right)+2 \tau\left(\operatorname{div} \mathbf{v}_{h}^{n} R_{h} T\left(t_{n}\right), r_{h}^{n}\right), \\
A_{3,2}=4 \tau\left(P_{h} \mathbf{u}\left(t_{n}\right) \nabla \varphi_{h}^{n}, r_{h}^{n}\right)+2 \tau\left(\operatorname{div} \mathbf{v}_{h}^{n} \varphi_{h}^{n}, r_{h}^{n}\right)
\end{gathered}
$$

and

$$
A_{3,3}=4 \tau\left(\left(P_{h} \mathbf{u}\left(t_{n}\right)-\mathbf{u}\left(t_{n}\right)\right) \nabla T\left(t_{n}\right), r_{h}^{n}\right)+2 \tau\left(\operatorname{div}\left(P_{h} \mathbf{u}\left(t_{n}\right)-\mathbf{u}\left(t_{n}\right)\right) T\left(t_{n}\right), r_{h}^{n}\right) .
$$

Therefore, we get the following inequalities,

$$
\begin{aligned}
\left|A_{3,1}\right| \leq & \frac{2\left(S_{4}^{0}\right)^{4} c_{4} \tau}{\varepsilon_{5}}\left|\mathbf{v}_{h}^{n}\right|_{H_{0}^{1}(\Omega)^{d}}^{2}\|T\|_{L^{\infty}\left(0, \hat{T} ; H^{1}(\Omega)\right)}^{2} \\
& +\frac{\left(S_{4}^{0}\right)^{4} c_{5} \tau}{\varepsilon_{6}}\left|\mathbf{v}_{h}^{n}\right|_{H_{0}^{1}(\Omega)^{d}}^{2}\|T\|_{L^{\infty}\left(0, \hat{T} ; H^{1}(\Omega)\right)}^{2} \\
& +2 \tau \varepsilon_{5}\left|r_{h}^{n}\right|_{H_{0}^{1}(\Omega)}^{2}+\tau \varepsilon_{6}\left|r_{h}^{n}\right|_{H_{0}^{1}(\Omega)}^{2}, \\
\left|A_{3,2}\right| \leq \quad & \frac{2\left(S_{4}^{0}\right)^{4} c_{6} \tau}{\varepsilon_{7}} h^{4}\|T\|_{L^{\infty}\left(0, \hat{T}: H^{3}(\Omega)\right)}^{2}\|\mathbf{u}\|_{L^{\infty}\left(0, \hat{T} ; H^{1}(\Omega)^{d}\right)}^{2} \\
& +\frac{\left(S_{4}^{0}\right)^{4} c_{7} \tau}{\varepsilon_{8}} h^{4}\|T\|_{L^{\infty}\left(0, \hat{T}: H^{3}(\Omega)\right)}^{2}\|T\|_{L^{\infty}\left(0, \hat{T} ; H^{1}(\Omega)\right)}^{2} \\
& +2 \tau \varepsilon_{7}\left|r_{h}^{n}\right|_{H_{0}^{1}(\Omega)}^{2}+\tau \varepsilon_{8}\left|r_{h}^{n}\right|_{H_{0}^{1}(\Omega)}^{2},
\end{aligned}
$$

and

$$
\begin{aligned}
\left|A_{3,3}\right| \leq & \frac{2\left(S_{4}^{0}\right)^{4} c_{8} \tau}{\varepsilon_{9}} h^{4}\|\mathbf{u}\|_{L^{\infty}\left(0, \hat{T}: H^{3}(\Omega)^{d}\right)}^{2}\|T\|_{L^{\infty}\left(0, \hat{T} ; H^{1}(\Omega)\right)}^{2} \\
& +\frac{\left(S_{4}^{0}\right)^{4} c_{9} \tau}{\varepsilon_{10}} h^{4}\|\mathbf{u}\|_{L^{\infty}\left(0, \hat{T}: H^{3}(\Omega)^{d}\right)}^{2}\|T\|_{L^{\infty}\left(0, \hat{T} ; H_{0}^{1}(\Omega)\right)}^{2} \\
& +2 \tau \varepsilon_{9}\left|r_{h}^{n}\right|_{H_{0}^{1}(\Omega)}^{2}+\tau \varepsilon_{10}\left|r_{h}^{n}\right|_{H_{0}^{1}(\Omega)}^{2} .
\end{aligned}
$$

Finally, by summing over $2 \leq n \leq m$, for all $m \leq N$, and using (2.6), we infer

$$
\begin{aligned}
& \left\|r_{h}^{m}\right\|_{L^{2}(\Omega)}^{2}+\left\|2 r_{h}^{m}-r_{h}^{m-1}\right\|_{L^{2}(\Omega)}^{2}+\sum_{n=2}^{m}\left\|\delta^{2} r_{h}^{n-1}\right\|_{L^{2}(\Omega)}^{2}+4 \sum_{n=2}^{m} \tau \alpha\left|r_{h}^{n}\right|_{H_{0}^{1}(\Omega)}^{2} \\
& \leq \xi_{1}\left(\tau^{4}+h^{4}\right)+\xi_{2} \sum_{n=2}^{m} \tau\left|r_{h}^{n}\right|_{H_{0}^{1}(\Omega)}^{2}+\xi_{3} \sum_{n=2}^{m} \tau\left|\mathbf{v}_{h}^{n}\right|_{H_{0}^{1}(\Omega)^{d}}^{2}+3\left\|r_{h}^{1}\right\|_{L^{2}(\Omega)}^{2}
\end{aligned}
$$

where $\xi_{i}(i=1,2,3)$ depend on $\varepsilon_{j}, j=1, \ldots, 10$. Then after applying the result of Theorem 5.6 and a suitable choice of $\varepsilon_{i}, i \in\{1, \ldots, 10\}\left(\xi_{2}=3 \alpha\right)$, we drive the following estimate

$$
\begin{aligned}
& \left\|r_{h}^{m}\right\|_{L^{2}(\Omega)}^{2}+\left\|2 r_{h}^{m}-r_{h}^{m-1}\right\|_{L^{2}(\Omega)}^{2}+\sum_{n=2}^{m}\left\|\delta^{2} r_{h}^{n-1}\right\|_{L^{2}(\Omega)}^{2}+\sum_{n=2}^{m} \tau \alpha\left|r_{h}^{n}\right|_{H_{0}^{1}(\Omega)}^{2} \\
& \quad \leq \hat{C}_{T}^{\prime}\left(\tau^{4}+h^{4}\right)+\hat{C}_{T}^{\prime \prime} \sum_{n=2}^{m} \tau\left|\mathbf{v}_{h}^{n}\right|_{H_{0}^{1}(\Omega)^{2}}^{2}
\end{aligned}
$$

where $\hat{C}_{T}^{\prime}$ and $\hat{C}_{T}^{\prime \prime}$ are constants that do not dependant on $\tau$ and $h$.

Theorem 5.8. Let $d=2,(\boldsymbol{u}, T, p)$ be the solution of Problem $(\mathrm{E})$ and $\left(\boldsymbol{u}_{h}^{n}, T_{h}^{n}, p_{h}^{n}\right)$ be the solution of Problem (5.8). If $\boldsymbol{u} \in L^{\infty}\left(0, T ; H^{3}(\Omega)^{2}\right), T \in L^{\infty}\left(0, T ; H^{3}(\Omega)\right), T^{\prime} \in L^{\infty}\left(0, T ; H^{2}(\Omega)\right)$ and $T^{(3)} \in$ $L^{2}\left(0, T ; L^{2}(\Omega)\right)$, there exists positive constants $\hat{C}_{\mathbf{u}}, \hat{C}_{\mathbf{u}}^{\prime}$ and $\hat{C}_{\mathbf{u}}^{\prime \prime}$ depending on a positive parameter $\tilde{\delta}_{1}$ 
such that for all $m \leq N$, we have for a time step $\tau$ sufficiently small,

$$
\begin{aligned}
& \left\|\mathbf{u}_{h}^{m}-P_{h} \mathbf{u}\left(t_{m}\right)\right\|_{L^{2}(\Omega)^{2}}^{2}+\left\|2\left(\mathbf{u}_{h}^{m}-P_{h} \mathbf{u}^{m}\right)-\left(\mathbf{u}_{h}^{m-1}-P_{h} \mathbf{u}\left(t_{m-1}\right)\right)\right\|_{L^{2}(\Omega)^{2}}^{2} \\
& +\sum_{n=2}^{m}\left\|\delta^{2}\left(\mathbf{u}_{h}^{n-1}-P_{h} \mathbf{u}\left(t_{n-1}\right)\right)\right\|_{L^{2}(\Omega)^{2}}^{2}+\sum_{n=2}^{m} \tau \hat{\nu}_{1}\left|\mathbf{u}_{h}^{n}-P_{h} \mathbf{u}\left(t_{n}\right)\right|_{H_{0}^{1}(\Omega)^{2}}^{2} \\
& \quad \leq \hat{C}_{\mathbf{u}}\left(h^{4}+\tau^{4}\right)+\hat{C}_{\mathbf{u}}^{\prime} \tilde{\delta}_{1} \sum_{n=2}^{m} \tau\left|T_{h}^{n}-T\left(t_{n}\right)\right|_{H_{0}^{1}(\Omega)}^{2}+\frac{\hat{C}_{\mathbf{u}}^{\prime \prime}}{\tilde{\delta}_{1}} \sum_{n=2}^{m} \tau\left\|T_{h}^{n}-T\left(t_{n}\right)\right\|_{L^{2}(\Omega)}^{2} .
\end{aligned}
$$

Proof. We denote by $\mathbf{v}_{h}=\mathbf{v}_{h}^{n}=\mathbf{u}_{h}^{n}-P_{h} \mathbf{u}\left(t_{n}\right) \in V_{h}$ and $\tilde{\varphi}_{h}^{n}=P_{h} \mathbf{u}\left(t_{n}\right)-\mathbf{u}\left(t_{n}\right)$. We first write the equation of the velocity by choosing $\mathbf{v}_{h}=\mathbf{v}_{h}^{n}$ in (Eds1), we obtain

$$
\begin{aligned}
& 2\left(3 \mathbf{u}_{h}^{n}-4 \mathbf{u}_{h}^{n-1}+\mathbf{u}_{h}^{n-2}, \mathbf{v}_{h}^{n}\right)+4 \tau\left(\nu\left(T_{h}^{n}\right) \nabla \mathbf{u}_{h}^{n}, \nabla \mathbf{v}_{h}^{n}\right)-4 \tau\left(p_{h}^{n}, \operatorname{div} \mathbf{v}_{h}^{n}\right)+4 \tau\left(\mathbf{u}_{h}^{n} \nabla \mathbf{u}_{h}^{n}, \mathbf{v}_{h}^{n}\right) \\
& \quad+2 \tau\left(\operatorname{div}\left(\mathbf{u}_{h}^{n}\right) \mathbf{u}_{h}^{n}, \mathbf{v}_{h}^{n}\right)=4 \tau\left(\mathbf{f}^{n}\left(T_{h}^{n}\right), \mathbf{v}_{h}^{n}\right) .
\end{aligned}
$$

In the other hand, we consider the first equation of (E) at $t=t_{n}$, we obtain by taking $\mathbf{v}=\mathbf{v}_{h}^{n}$

$$
\begin{aligned}
& 4 \tau\left(\mathbf{u}^{\prime}\left(t_{n}\right), \mathbf{v}_{h}^{n}\right)+4 \tau\left(\nu\left(T\left(t_{n}\right)\right) \nabla \mathbf{u}\left(t_{n}\right), \nabla \mathbf{v}_{h}^{n}\right)-4 \tau\left(p\left(t_{n}\right), \operatorname{div} \mathbf{v}_{h}^{n}\right) \\
& \quad+4 \tau\left(\mathbf{u}\left(t_{n}\right) \nabla \mathbf{u}\left(t_{n}\right), \mathbf{v}_{h}^{n}\right)+2 \tau\left(\operatorname{div}\left(\mathbf{u}\left(t_{n}\right)\right) \mathbf{u}\left(t_{n}\right), \mathbf{v}_{h}^{n}\right)=4 \tau\left(\mathbf{f}\left(T\left(t_{n}\right)\right), \mathbf{v}_{h}^{n}\right) .
\end{aligned}
$$

We substruct (5.41) and (5.42), and insert $\pm 2\left(3 \mathbf{u}_{h}^{n}-4 \mathbf{u}_{h}^{n-1}+\mathbf{u}_{h}^{n-2}, \mathbf{v}_{h}^{n}\right), \pm 2\left(3 P_{h} \mathbf{u}\left(t_{n}\right)-4 P_{h} \mathbf{u}\left(t_{n-1}\right)+\right.$ $\left.P_{h} \mathbf{u}\left(t_{n-2}\right), \mathbf{v}_{h}^{n}\right)$ and $\pm 4 \tau\left(\nu\left(T_{h}^{n}\right) \nabla P_{h} \mathbf{u}\left(t_{n}\right), \nabla \mathbf{v}_{h}^{n}\right)$. We obtain

$$
\begin{aligned}
& \left\|\mathbf{v}_{h}^{n}\right\|_{L^{2}(\Omega)^{2}}^{2}+\left\|2 \mathbf{v}_{h}^{n}-\mathbf{v}_{h}^{n-1}\right\|_{L^{2}(\Omega)^{2}}^{2}+\left\|\delta^{2} \mathbf{v}_{h}^{n-1}\right\|_{L^{2}(\Omega)^{2}}^{2} \\
& -\left\|\mathbf{v}_{h}^{n-1}\right\|_{L^{2}(\Omega)^{2}}^{2}-\left\|2 \mathbf{v}_{h}^{n-1}-\mathbf{v}_{h}^{n-2}\right\|_{L^{2}(\Omega)^{2}}^{2}+4 \tau \hat{\nu}_{1}\left|\mathbf{v}_{h}^{n}\right|_{H_{0}^{1}(\Omega)^{2}}^{2} \\
& \leq \quad\left|2\left(3 \tilde{\varphi}_{h}^{n}-4 \tilde{\varphi}_{h}^{n-1}+\tilde{\varphi}_{h}^{n-2}, \mathbf{v}_{h}^{n}\right)\right|+\left|4 \tau\left(\nu\left(T_{h}^{n}\right) \nabla P_{h} \mathbf{u}\left(t_{n}\right), \nabla \mathbf{v}_{h}^{n}\right)-4 \tau\left(\nu\left(T\left(t_{n}\right)\right) \nabla \mathbf{u}\left(t_{n}\right), \nabla \mathbf{v}_{h}^{n}\right)\right| \\
& \quad+\left|4 \tau\left(\mathbf{u}_{h}^{n} \nabla \mathbf{u}_{h}^{n}, \mathbf{v}_{h}^{n}\right)+2 \tau\left(\operatorname{div}\left(\mathbf{u}_{h}^{n}\right) \mathbf{u}_{h}^{n}, \mathbf{v}_{h}^{n}\right)-4 \tau\left(\mathbf{u}\left(t_{n}\right) \nabla \mathbf{u}\left(t_{n}\right), \mathbf{v}_{h}^{n}\right)-2 \tau\left(\operatorname{div}\left(\mathbf{u}\left(t_{n}\right)\right) \mathbf{u}\left(t_{n}\right), \mathbf{v}_{h}^{n}\right)\right| \\
& \quad+\left|4 \tau\left(p_{h}^{n}-p\left(t_{n}\right), \operatorname{div} \mathbf{v}_{h}^{n}\right)\right|+\left|4 \tau\left(\mathbf{f}_{1}\left(T_{h}^{n}\right), \mathbf{v}_{h}^{n}\right)-4 \tau\left(\mathbf{f}\left(T\left(t_{n}\right)\right), \mathbf{v}_{h}^{n}\right)\right|+\left|\tilde{R}_{1}\right|,
\end{aligned}
$$

where

$$
\tilde{R}_{1}=\left(\mathbf{u}^{\prime}(t)-\frac{3 \mathbf{u}(t)-4 \mathbf{u}(t-\tau)+\mathbf{u}(t-2 \tau)}{2 \tau}, \mathbf{v}_{h}\right) .
$$

Let us study the terms of right hand side of (5.43) denoted by $\tilde{A}_{i}, i \in\{1, \ldots, 5\}$. Then we have, by using Taylor's formula

$$
\frac{3 \tilde{\varphi}_{h}^{n}-4 \tilde{\varphi}_{h}^{n-1}+\tilde{\varphi}_{h}^{n-2}}{2 \tau}=P_{h} \mathbf{u}^{\prime}\left(t_{n}\right)-u^{\prime}\left(t_{n}\right)+\tilde{R}_{2},
$$

where

$$
\left|\tilde{R}_{2}\right| \leq \frac{\tau^{\frac{3}{2}}}{2 \sqrt{3}}\left\|\left(P_{h} \mathbf{u}^{(3)}-\mathbf{u}^{(3)}\right)(x)\right\|_{L^{2}\left(t_{n-2}, t_{n}\right)} .
$$

Since $P_{h}$ is stable by norm $L^{2}$ (cf. [8]), then we obtain

$$
\begin{aligned}
\left|\tilde{A}_{1}\right| \leq & \frac{2 c_{1} h^{4}}{\tilde{\varepsilon_{1}}}\left\|\mathbf{u}^{\prime}\right\|_{L^{\infty}\left(0, \hat{T} ; H^{2}(\Omega)^{2}\right)}^{2}+2 \tau \tilde{\varepsilon}_{1} S_{2}^{2}\left|\mathbf{v}_{h}^{n}\right|_{H_{0}^{1}(\Omega)^{2}}^{2} \\
& +\frac{\tau^{4} c_{2}}{\tilde{\varepsilon}_{2}}\left\|\mathbf{u}^{(3)}\right\|_{L^{2}\left(0, \hat{T} ; L^{2}(\Omega)^{2}\right)}^{2}+\tau \tilde{\varepsilon}_{2} S_{2}^{2}\left|\mathbf{v}_{h}^{n}\right|_{H_{0}^{1}(\Omega)^{2}}^{2}
\end{aligned}
$$

For the terme $\left|\tilde{A}_{2}\right|$, we get

$$
\begin{aligned}
\left|\tilde{A}_{2}\right| \leq & \frac{2\left(\hat{\nu}_{1}\right)^{2} c_{3} h^{4} \tau}{\tilde{\varepsilon}_{3}}\|\mathbf{u}\|_{L^{\infty}\left(0, \hat{T} ; H^{3}(\Omega)^{2}\right)}^{2}+2 \tau \tilde{\varepsilon}_{3}\left|\mathbf{v}_{h}^{n}\right|_{H_{0}^{1}(\Omega)^{2}}^{2} \\
& +\frac{2 \tau\left(\nu^{*}\right)^{4}}{\tilde{\varepsilon}_{4} \tilde{\delta}_{1}}\|\mathbf{u}\|_{L^{\infty}\left(0, \hat{T} ; W^{1,4}(\Omega)^{2}\right)}^{4}\left\|T_{h}^{n}-T\left(t_{n}\right)\right\|_{L^{2}(\Omega)}^{2} \\
& +\frac{\tau \tilde{\delta}_{1}}{\tilde{\varepsilon}_{4}}\left|T_{h}^{n}-T\left(t_{n}\right)\right|_{H_{0}^{1}(\Omega)}^{2}+\tau \tilde{\varepsilon}_{4}\left|\mathbf{v}_{h}^{n}\right|_{H_{0}^{1}(\Omega)^{2}}^{2} .
\end{aligned}
$$


We add and subsruct $4 \tau d_{\mathbf{u}}\left(\mathbf{u}_{h}^{n}, P_{h} \mathbf{u}\left(t_{n}\right), \mathbf{v}_{h}^{n}\right), 4 \tau d_{\mathbf{u}}\left(P_{h} \mathbf{u}\left(t_{n}\right), P_{h} \mathbf{u}\left(t_{n}\right), \mathbf{v}_{h}^{n}\right)$ and $4 \tau d_{\mathbf{u}}\left(\mathbf{u}\left(t_{n}\right), P_{h} \mathbf{u}\left(t_{n}\right), \mathbf{v}_{h}^{n}\right)$ in the $\tilde{A}_{3}$ to obtain

$$
\begin{aligned}
\left|\tilde{A}_{3}\right|= & 4 \tau\left(\mathbf{v}_{h}^{n} \nabla P_{h} \mathbf{u}\left(t_{n}\right), \mathbf{v}_{h}^{n}\right)+2 \tau\left(\operatorname{div}\left(\mathbf{v}_{h}^{n}\right) P_{h} \mathbf{u}\left(t_{n}\right), \mathbf{v}_{h}^{n}\right) \\
& +4 \tau\left(\left(P_{h} \mathbf{u}\left(t_{n}\right)-\mathbf{u}\left(t_{n}\right)\right) \nabla P_{h} \mathbf{u}\left(t_{n}\right), \mathbf{v}_{h}^{n}\right)+2 \tau\left(\operatorname{div}\left(P_{h} \mathbf{u}\left(t_{n}\right)-\mathbf{u}\left(t_{n}\right)\right) P_{h} \mathbf{u}\left(t_{n}\right), \mathbf{v}_{h}^{n}\right) \\
& +4 \tau\left(\mathbf{u}\left(t_{n}\right) \nabla\left(P_{h} \mathbf{u}\left(t_{n}\right)-\mathbf{u}\left(t_{n}\right)\right), \mathbf{v}_{h}^{n}\right)+2 \tau\left(\operatorname{div}\left(\mathbf{u}\left(t_{n}\right)\right)\left(P_{h} \mathbf{u}\left(t_{n}\right)-\mathbf{u}\left(t_{n}\right)\right) \mathbf{u}\left(t_{n}\right), \mathbf{v}_{h}^{n}\right) .
\end{aligned}
$$

We denote by $\tilde{A}_{3,1}, \tilde{A}_{3,2}$ and $\tilde{A}_{3,3}$ the terms of the right hand side of (5.48).

We have by using $(2.2)$,

$$
\begin{aligned}
\left|\left(\operatorname{div}\left(\mathbf{v}_{h}^{n}\right) P_{h} \mathbf{u}\left(t_{n}\right), \mathbf{v}_{h}^{n}\right)\right| & \leq c_{4}\left|\mathbf{v}_{h}^{n}\right|_{H^{1}(\Omega)}\left|\mathbf{u}\left(t_{n}\right)\right|_{L^{4}(\Omega)}\left|\mathbf{v}_{h}^{n}\right|_{L^{4}(\Omega)} \\
& \leq c_{5}\left|\mathbf{v}_{h}^{n}\right|_{H^{1}(\Omega)}^{3 / 2}\left|\mathbf{u}\left(t_{n}\right)\right|_{L^{2}(\Omega)}^{1 / 2},
\end{aligned}
$$

and then by using the relation $a b \leq \frac{a^{4}}{4}+\frac{b^{4 / 3}}{4 / 3}$, we get the following bound,

$$
\begin{aligned}
\left|\tilde{A}_{3,1}\right| \leq & 2 \sqrt{2} \tau c_{6} \tilde{\varepsilon}_{5}\|\mathbf{u}\|_{L^{\infty}\left(0, \hat{T} ; H_{0}^{1}(\Omega)^{2}\right)}\left|\mathbf{v}_{h}^{n}\right|_{H_{0}^{1}(\Omega)^{2}}^{2}+\frac{2 \sqrt{2} \tau}{\tilde{\varepsilon}_{5}}\|\mathbf{u}\|_{L^{\infty}\left(0, \hat{T} ; H_{0}^{1}(\Omega)^{2}\right)}\left\|\mathbf{v}_{h}^{n}\right\|_{L^{2}(\Omega)^{2}}^{2} \\
& +\frac{S_{4}^{0} c_{7} \tau \tilde{\varepsilon}_{6}}{2^{\frac{3}{4}}}\|\mathbf{u}\|_{L^{\infty}\left(0, \hat{T} ; H_{0}^{1}(\Omega)^{2}\right)}\left|\mathbf{v}_{h}^{n}\right|_{H_{0}^{1}(\Omega)^{2}}^{2}+\frac{3 S_{4}^{0} c_{8} \tau}{2^{\frac{3}{4} \tilde{\varepsilon}_{6}}}\|\mathbf{u}\|_{L^{\infty}\left(0, \hat{T} ; H_{0}^{1}(\Omega)^{2}\right)}\left\|\mathbf{v}_{h}^{n}\right\|_{L^{2}(\Omega)^{2}}^{2}
\end{aligned}
$$

The relation

$$
2 \tau\left(\operatorname{div}\left(\mathbf{v}_{h}^{n}\right) P_{h} \mathbf{u}\left(t_{n}\right), \mathbf{v}_{h}^{n}\right)=-4 \tau\left(\mathbf{v}_{h}^{n} \nabla P_{h} \mathbf{u}\left(t_{n}\right), \mathbf{v}_{h}^{n}\right)-4 \tau\left(\mathbf{v}_{h}^{n} \nabla \mathbf{v}_{h}^{n}, P_{h} \mathbf{u}\left(t_{n}\right)\right)
$$

allows us to get the inequality

$$
\left|\tilde{A}_{3,2}\right| \leq \frac{4 \tau\left(S_{4}^{0}\right)^{4} c_{9}}{\tilde{\varepsilon}_{7}} h^{4}\|\mathbf{u}\|_{L^{\infty}\left(0, \hat{T} ; H_{0}^{1}(\Omega)^{2}\right)}^{2}\|\mathbf{u}\|_{L^{\infty}\left(0, \hat{T} ; H^{3}(\Omega)^{2}\right)}^{2}+4 \tau \tilde{\varepsilon}_{7}\left|\mathbf{v}_{h}^{n}\right|_{H_{0}^{1}(\Omega)^{2}}^{2}
$$

and

$$
\left|\tilde{A}_{3,3}\right| \leq \frac{4 \tau\left(S_{4}^{0}\right)^{4} c_{10}}{\tilde{\varepsilon}_{8}} h^{4}\|\mathbf{u}\|_{L^{\infty}\left(0, \hat{T} ; H_{0}^{1}(\Omega)^{2}\right)}^{2}\|\mathbf{u}\|_{L^{\infty}\left(0, \hat{T} ; H^{3}(\Omega)^{2}\right)}^{2}+4 \tau \tilde{\varepsilon}_{8}\left|\mathbf{v}_{h}^{n}\right|_{H_{0}^{1}(\Omega)^{2}}^{2} .
$$

For the pressure, we have

$$
\left|\tilde{A}_{4}\right| \leq \frac{4 \tau c_{11} h^{4}}{\tilde{\varepsilon}_{9}}\|p\|_{L^{\infty}\left(0, \hat{T} ; H^{2}(\Omega)\right)}^{2}+2 \tau \tilde{\varepsilon}_{9}\left|\mathbf{v}_{h}^{n}\right|_{H_{0}^{1}(\Omega)^{2}}^{2} .
$$

The last terme is bounded as follow

$$
\left|\tilde{A}_{5}\right| \leq \frac{2 \tau\left(c_{\mathbf{f}_{1}}^{*}\right)^{2}\left(S_{4}^{0}\right)^{2}}{\tilde{\varepsilon}_{10}}\left\|T_{h}^{n}-T\left(t_{n}\right)\right\|_{L^{2}(\Omega)}^{2}+2 \tau \tilde{\varepsilon}_{10}\left|\mathbf{v}_{h}^{n}\right|_{H_{0}^{1}(\Omega)^{2}}^{2} .
$$

By summing over $2 \leq n \leq m$, for all $m \leq N$, we get the estimation

$$
\begin{aligned}
& \left\|\mathbf{v}_{h}^{m}\right\|_{L^{2}(\Omega)^{2}}^{2}+\left\|2 \mathbf{v}_{h}^{m}-\mathbf{v}_{h}^{m-1}\right\|_{L^{2}(\Omega)^{2}}^{2}+\sum_{n=2}^{m}\left\|\delta^{2} \mathbf{v}_{h}^{n-1}\right\|_{L^{2}(\Omega)^{2}}^{2}-3\left\|\mathbf{v}_{h}^{1}\right\|_{L^{2}(\Omega)^{2}}^{2}+4 \sum_{n=2}^{m} \tau \hat{\nu}_{1}\left|\mathbf{v}_{h}^{n}\right|_{H_{0}^{1}(\Omega)^{2}}^{2} \\
& \leq \tilde{\xi}_{1}\left(h^{4}+\tau^{4}\right)+\sum_{n=2}^{m} \frac{\tau \tilde{\delta}_{1}}{\tilde{\varepsilon_{4}}}\left|T_{h}^{n}-T\left(t_{n}\right)\right|_{H_{0}^{1}(\Omega)}^{2}+\sum_{n=2}^{m} \frac{\tilde{\xi}_{2} \tau}{\tilde{\delta}_{1}}\left\|T_{h}^{n}-T\left(t_{n}\right)\right\|_{L^{2}(\Omega)}^{2} \\
& \quad+\sum_{n=2}^{m} \tilde{\xi}_{3} \tau\left\|\mathbf{v}_{h}^{n}\right\|_{L^{2}(\Omega)^{2}}^{2}+\sum_{n=2}^{m} \tilde{\xi}_{4} \tau\left|\mathbf{v}_{h}^{n}\right|_{H_{0}^{1}(\Omega)^{2}}^{2},
\end{aligned}
$$

where $\tilde{\xi}_{i}, i=1, \ldots, 4$ depend of $\tilde{\varepsilon}_{i}, i=1, \ldots, 10$.

Therefor, by collecting the above bounds, using Theorem 5.6 and choosing suitable values of $\tilde{\varepsilon}_{i}, i \in$ $\{1, . ., 10\}$, we obtain the following result:

$$
\begin{aligned}
& \left\|\mathbf{v}_{h}^{m}\right\|_{L^{2}(\Omega)^{2}}^{2}+\left\|2 \mathbf{v}_{h}^{m}-\mathbf{v}_{h}^{m-1}\right\|_{L^{2}(\Omega)^{2}}^{2}+\sum_{n=2}^{m}\left\|\delta^{2} \mathbf{v}_{h}^{n-1}\right\|_{L^{2}(\Omega)^{2}}^{2}+\sum_{n=2}^{m} \tau \hat{\nu}_{1}\left|\mathbf{v}_{h}^{n}\right|_{H_{0}^{1}(\Omega)^{2}}^{2} \\
& \leq \hat{C}_{\mathbf{u}}\left(h^{4}+\tau^{4}\right)+\hat{C}_{\mathbf{u}}^{\prime} \tilde{\delta}_{1} \sum_{n=2}^{m} \tau\left|T_{h}^{n}-T\left(t_{n}\right)\right|_{H_{0}^{1}(\Omega)}^{2}+\frac{\hat{C}_{\mathbf{u}}^{\prime \prime}}{\tilde{\delta}_{1}} \sum_{n=2}^{m} \tau\left\|T_{h}^{n}-T\left(t_{n}\right)\right\|_{L^{2}(\Omega)}^{2}+\hat{C}_{\mathbf{u}}^{\prime \prime \prime} \sum_{n=2}^{m} \tau\left\|\mathbf{v}_{h}^{n}\right\|_{L^{2}(\Omega)^{2}}^{2} .
\end{aligned}
$$


After writing

$$
\hat{C}_{\mathbf{u}}^{\prime \prime \prime} \sum_{n=2}^{m} \tau\left\|\mathbf{v}_{h}^{n}\right\|_{L^{2}(\Omega)^{2}}^{2}=\hat{C}_{\mathbf{u}}^{\prime \prime \prime} \sum_{n=2}^{m-1} \tau\left\|\mathbf{v}_{h}^{n}\right\|_{L^{2}(\Omega)^{2}}^{2}+\hat{C}_{\mathbf{u}}^{\prime \prime \prime} \tau\left\|\mathbf{v}_{h}^{m}\right\|_{L^{2}(\Omega)^{2}}^{2},
$$

Using Gronwall's lemma for $\tau \leq \frac{1}{2 \hat{C}_{\mathbf{u}}^{\prime \prime}}$, we infer

$$
\begin{gathered}
\left\|\mathbf{v}_{h}^{m}\right\|_{L^{2}(\Omega)^{2}}^{2}+\left\|2 \mathbf{v}_{h}^{m}-\mathbf{v}_{h}^{m-1}\right\|_{L^{2}(\Omega)^{2}}^{2}+\sum_{n=2}^{m}\left\|\delta \mathbf{v}_{h}^{n-1}\right\|_{L^{2}(\Omega)^{2}}^{2}+\sum_{n=2}^{m} \tau \hat{\nu}_{1}\left|\mathbf{v}_{h}^{n}\right|_{H_{0}^{1}(\Omega)^{2}}^{2} \\
\leq \hat{C}_{\mathbf{u}}\left(h^{4}+\tau^{4}\right)+\hat{C}_{\mathbf{u}} \tilde{\delta}_{1} \sum_{n=2}^{m} \tau\left|T_{h}^{n}-T\left(t_{n}\right)\right|_{H_{0}^{1}(\Omega)}^{2}+\frac{\hat{C}_{\mathbf{u}}^{\prime \prime}}{\tilde{\delta}_{1}} \sum_{n=2}^{m} \tau\left\|T_{h}^{n}-T\left(t_{n}\right)\right\|_{L^{2}(\Omega)}^{2} .
\end{gathered}
$$

Theorem 5.9. Under the assumption of Theoreme 5.7 and 5.8, there exists constants $\hat{C}_{\mathbf{u}}$ and $\hat{C}_{T}$ independent of $\tau$ and $h$, such that for $\tau$ sufficiently small, we have

$$
\begin{aligned}
\sup _{2 \leq n \leq N} & \left\|T_{h}^{n}-R_{h} T\left(t_{n}\right)\right\|_{L^{2}(\Omega)}^{2}+\sup _{2 \leq n \leq N} \| 2\left(T_{h}^{n}-R_{h} T\left(t_{n}\right)\right)-\left(T_{h}^{n-1}-R_{h} T\left(t_{n-1}\right) \|_{L^{2}(\Omega)}^{2}\right. \\
& +\sum_{n=2}^{N-1} \| \delta^{2}\left(T_{h}^{n-1}-R_{h} T\left(t_{n-1}\right) \|_{L^{2}(\Omega)}^{2}+\sum_{n=2}^{N} \frac{\tau \alpha}{2}\left|T_{h}^{n}-R_{h} T\left(t_{n}\right)\right|_{H_{0}^{1}(\Omega)}^{2} \leq \hat{C}_{T}\left(\tau^{4}+h^{4}\right)\right.
\end{aligned}
$$

and

$$
\begin{aligned}
\sup _{2 \leq n \leq N} & \left\|\mathbf{u}_{h}^{n}-P_{h} \mathbf{u}\left(t_{n}\right)\right\|_{L^{2}(\Omega)^{2}}^{2}+\sup _{2 \leq n \leq N}\left\|2\left(\mathbf{u}_{h}^{n}-P_{h} \mathbf{u}\left(t_{n}\right)\right)-\left(\mathbf{u}_{h}^{n-1}-P_{h} \mathbf{u}\left(t_{n-1}\right)\right)\right\|_{L^{2}(\Omega)^{2}}^{2} \\
& +\sum_{n=2}^{N-1}\left\|\delta^{2}\left(\mathbf{u}_{h}^{n-1}-P_{h} \mathbf{u}\left(t_{n-1}\right)\right)\right\|_{L^{2}(\Omega)^{2}}^{2}+\sum_{n=2}^{N} \frac{\tau \hat{\nu}_{1}}{2}\left|\mathbf{u}_{h}^{n}-P_{h} \mathbf{u}\left(t_{n}\right)\right|_{H_{0}^{1}(\Omega)^{2}}^{2} \leq \hat{C}_{\mathbf{u}}\left(\tau^{4}+h^{4}\right)
\end{aligned}
$$

Proof. We denote by $\mathbf{v}_{h}^{n}=\mathbf{u}_{h}^{n}-P_{h} \mathbf{u}\left(t_{n}\right)$ and $r_{h}^{n}=T_{h}^{n}-R_{h} T\left(t_{n}\right)$. We combine the results of Theorems 5.7 and 5.8 , insert $\pm R_{h} T\left(t_{n}\right)$, consider $T \in L^{\infty}\left(0, \hat{T} ; H^{2}(\Omega)\right)$ and choose $\tilde{\delta}_{1}=\frac{\alpha \hat{\nu}_{1}}{4 \hat{C}_{T}^{\prime \prime} \hat{C}_{\mathbf{u}}}$, to obtain

$$
\begin{aligned}
& \left\|r_{h}^{m}\right\|_{L^{2}(\Omega)}^{2}+\left\|2 r_{h}^{m}-r_{h}^{m-1}\right\|_{L^{2}(\Omega)}^{2}+\sum_{n=2}^{m}\left\|\delta^{2} r_{h}^{n-1}\right\|_{L^{2}(\Omega)}^{2}+\frac{1}{2} \sum_{n=2}^{m} \tau \alpha\left|r_{h}^{n}\right|_{H_{0}^{1}(\Omega)}^{2} \\
& \quad \leq \hat{C}_{T}^{\prime}\left(\tau^{4}+h^{4}\right)+C_{r} \sum_{n=2}^{m} \tau\left\|r_{h}^{n}\right\|_{L^{2}(\Omega)}^{2} .
\end{aligned}
$$

We observe that

$$
\left\|r_{h}^{m}\right\|_{L^{2}(\Omega)}^{2} \leq 2\left(\left\|\delta^{2} r_{h}^{m}\right\|_{L^{2}(\Omega)}^{2}+\left\|2 r_{h}^{m-1}-r_{h}^{m-2}\right\|_{L^{2}(\Omega)}^{2}\right)
$$

and consider $\tau$ sufficiently small such that, after applying Gronwall's lemma, we obtain

$$
\begin{aligned}
\sup _{2 \leq n \leq N} & \left\|r_{h}^{n}\right\|_{L^{2}(\Omega)}^{2}+\sup _{2 \leq n \leq N}\left\|2 r_{h}^{n}-r_{h}^{n-1}\right\|_{L^{2}(\Omega)}^{2}+\sum_{n=2}^{N-1}\left\|\delta^{2} r_{h}^{n-1}\right\|_{L^{2}(\Omega)}^{2} \\
& +\sum_{n=2}^{N} \frac{\tau \alpha}{2}\left|r_{h}^{n}\right|_{H_{0}^{1}(\Omega)}^{2} \leq \hat{C}_{T}\left(\tau^{4}+h^{4}\right)
\end{aligned}
$$

By considering the triangle inequality

$$
\left\|T_{h}^{n}-T\left(t_{n}\right)\right\|_{L^{2}(\Omega)} \leq\left\|T_{h}^{n}-R_{h} T\left(t_{n}\right)\right\|_{L^{2}(\Omega)}+\left\|R_{h} T\left(t_{n}\right)-T\left(t_{n}\right)\right\|_{L^{2}(\Omega)}
$$

we get (5.56). We deduce the bound (5.57) by applying theorem 5.8.

\section{NUMERICAL RESULTAS}

To validate the theoretical results, we perform several numerical simulations using the FreeFem ++ software (see [10]). We begin with a simple test case where we show comparisons with the exact solution for the first order schemes (Eds1) and (Eds2), and the second order scheme (5.8). 
6.1. First academic test case. We consider the square $\Omega=] 0,3\left[{ }^{2}\right.$ and the time interval $[0, \hat{T}]=[0,3]$. Each edge is divided into $N$ equal segments so that $\Omega$ is divided into $2 N^{2}$ triangles. The time step is considered as $\tau=\frac{\hat{T}}{N}$, so $h=\frac{3}{N}$ and $\tau$ are equal. In this section, we consider $\mathbf{f}_{1}(x, T)=0$.

We consider the manufactured solution

$$
(\mathbf{u}, p, T)=\left(t e^{t} \operatorname{curl} \psi, p, T\right),
$$

where $\psi, p$ and $T$ are defined as follows

$$
\begin{aligned}
& \psi(x, y)=e^{-30\left((x-1)^{2}+(y-1)^{2}\right),} \\
& p(x, t)=t \sin t \cos \frac{\pi}{3} x \cos \frac{\pi}{3} y, \\
& T(x, t)=\sin t x^{2}(x-3)^{2} y^{2}(y-3)^{2},
\end{aligned}
$$

In this section, we consider three cases for the viscosity: $\nu(T)=T+1, \nu(T)=\sin T+2$ and $\nu(T)=$ $e^{-T}+\frac{1}{10}$ and we define the following relative error between the exact and numerical solution

$$
e r r=\left(\frac{\sum_{n=1}^{M} \tau\left|\mathbf{u}_{h}^{n+1}-\mathbf{u}_{h}^{n}\right|_{H^{1}(\Omega)^{2}}^{2}}{\sum_{n=1}^{M} \tau\left|\mathbf{u}\left(t_{n}\right)\right|_{H^{1}(\Omega)^{2}}^{2}}+\frac{\sum_{n=1}^{M} \tau\left|p_{h}^{n+1}-p\left(t_{n}\right)\right|_{L^{2}(\Omega)}^{2}}{\sum_{n=1}^{M} \tau\left|p\left(t_{n}\right)\right|_{L^{2}(\Omega)}^{2}}+\frac{\sum_{n=1}^{M} \tau\left|T_{h}^{n+1}-T\left(t_{n}\right)\right|_{H^{1}(\Omega)}^{2}}{\sum_{n=1}^{M} \tau\left|T\left(t_{n}\right)\right|_{H^{1}(\Omega)}^{2}}\right)^{1 / 2} .
$$

\subsubsection{First order schemes.}

In this section, we study the schemes (Eds1) and (Eds2). Figures 1 and 2 (respectively (3 and 4) and (5 and 6)) show a comparison between the numerical and exact velocity (respectively the pressure and the heat) for $\nu(T)=T+1$. Same comparisons are obtained for the other cases of the viscosity.

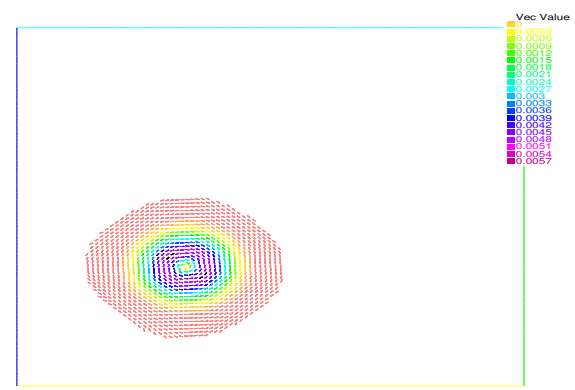

FiguRE 1. Numerical velocity for $\nu(T)=T+1$ and $N=100$.

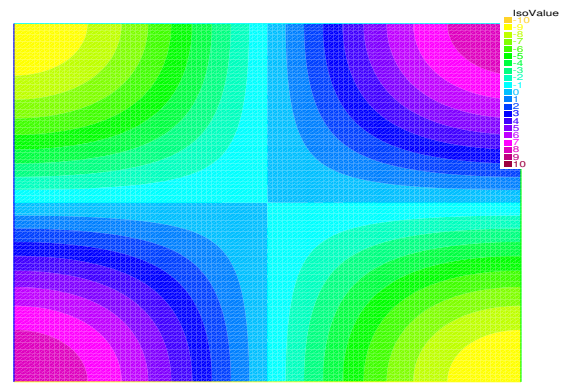

FIgURE 3. Numerical pressure for $\nu(T)=T+1$ and $N=100$.

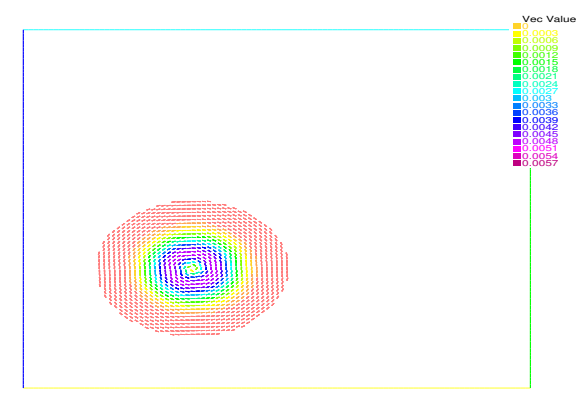

FIgURE 2. Exact velocity for $\nu(T)=T+1$ and $N=100$.

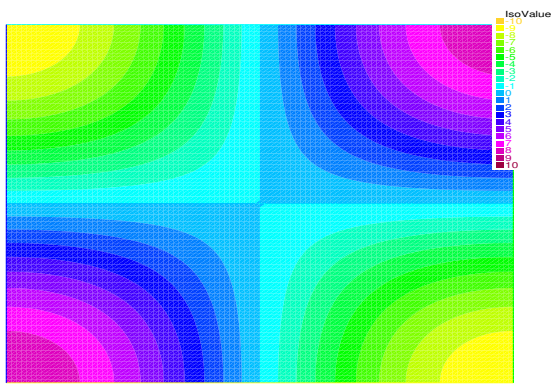

Figure 4. Exact pressure for $\nu(T)=T+1$ and $N=100$.

Table 1 shows the slope of the error curve (in logarithmic scale and for $N$ going from 60 to 120) corresponding to the different cases of $\nu(T)$ and the schemes (Eds1) and (Eds2): 


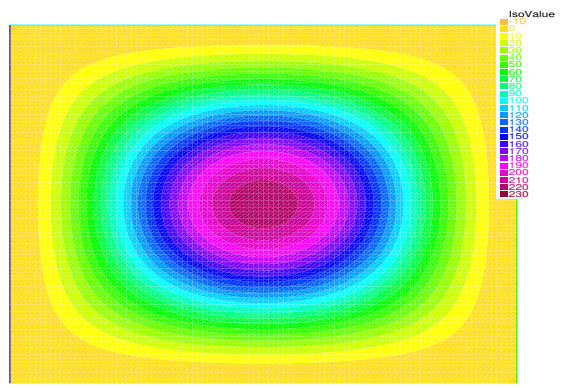

Figure 5. Numerical heat for $\nu(T)=T+1$ and $N=100$.

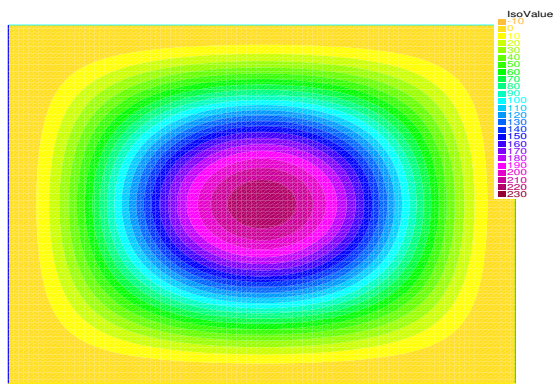

Figure 6. Exact heat for $\nu(T)=T+1$ and $N=100$.

\begin{tabular}{|c|c|c|}
\hline$\nu(T)$ & $($ Eds1) & $($ Eds2) \\
\hline \hline$T+1$ & 1,003 & 1,003 \\
\hline $\sin (T)+2$ & 0,984 & 0,941 \\
\hline$e^{-T}+\frac{1}{10}$ & 0,9841 & 0,9838 \\
\hline
\end{tabular}

TABLE 1. The slop of the error.

Remark 6.1. Note that the results obtained in Table 1 confirm those obtained in Theorem 4.7

\subsubsection{Second order scheme.}

In this section, we study (5.8) which is a nonlinear second order scheme and can be represented at each time step as $F(w)=0$, where $w=\left(\mathbf{u}_{h}^{n}, p_{h}^{n}, T_{h}^{n}\right)$ and

$$
F\left(w^{n}\right)=\left(\begin{array}{c}
\frac{3 \mathbf{u}_{h}^{n}-4 \mathbf{u}_{h}^{n-1}+\mathbf{u}_{h}^{n-2}}{2 \tau}+\mathbf{u}_{h}^{n} \nabla \mathbf{u}_{h}^{n}-\nu\left(T_{h}^{n}\right) \Delta \mathbf{u}_{h}^{n}+\nabla p_{h}^{n}+\frac{1}{2} \operatorname{div}\left(\mathbf{u}_{h}^{n}\right) \mathbf{u}_{h}^{n}-\mathbf{f}_{0}^{n} \\
\frac{3 T_{h}^{n}-4 T_{h}^{n-1}+T_{h}^{n-2}}{2 \tau}+\mathbf{u}_{h}^{n} \nabla T_{h}^{n}-\alpha \Delta T_{h}^{n}+\frac{1}{2} \operatorname{div}\left(\mathbf{u}_{h}^{n}\right) T_{h}^{n}-g^{n}
\end{array}\right) .
$$

We solve the equation $F(w)=0$ by using the following iterative Newton method: let $w^{0}=\left(\mathbf{u}_{h}^{n-1}, p_{h}^{n-1}, T_{h}^{n-1}\right)$. At each iteration $k$, knowing $w^{k-1}$, we solve the following system

$$
D_{w} F\left(w^{k-1}\right)\left(w^{k}-w^{k-1}\right)=-F\left(w^{k-1}\right),
$$

to get $w^{k}=\left(\mathbf{u}^{k}, p^{k}, T^{k}\right)$. Note that $D_{w} F$ is the derivative of $F$.

By denoting $\left(\mathbf{u}_{w}, p_{w}, T_{w}\right)=w^{k}-w^{k-1}$, system (6.4) can be written, for all $\mathbf{v}_{h} \in X_{n h}$ and $r_{h} \in Y_{n h}$, as

$$
\begin{aligned}
( & \left.\frac{3 \mathbf{u}_{w}}{2 \tau}, \mathbf{v}_{h}\right)+\left(\mathbf{u}_{w} \nabla \mathbf{u}^{k-1}, \mathbf{v}_{h}\right)+\left(\mathbf{u}^{k-1} \nabla \mathbf{u}_{w}, \mathbf{v}_{h}\right)+\left(\nu\left(T^{k-1}\right) \nabla \mathbf{u}_{w}, \nabla \mathbf{v}_{h}\right) \\
& +\left(\frac{d \nu}{d T}\left(T^{k-1}\right) T_{w} \nabla \mathbf{u}^{k-1}, \nabla \mathbf{v}_{h}\right)-\left(p_{w}, \operatorname{div} \mathbf{v}_{h}\right)+\frac{1}{2}\left(\operatorname{div}\left(\mathbf{u}_{w}\right) \mathbf{u}^{k-1}, \mathbf{v}_{h}\right)+\frac{1}{2}\left(\operatorname{div}\left(\mathbf{u}^{k-1}\right) \mathbf{u}_{w}, \mathbf{v}_{h}\right) \\
= & -\left(\frac{3 \mathbf{u}^{k-1}-4 \mathbf{u}_{h}^{n-1}+\mathbf{u}_{h}^{n-2}}{2 \tau}, \mathbf{v}_{h}\right)-\left(\mathbf{u}^{k-1} \nabla \mathbf{u}^{k-1}, \mathbf{v}_{h}\right)+\left(p^{k-1}, \operatorname{div} \mathbf{v}_{h}\right) \\
& -\left(\nu\left(T_{h}^{k-1}\right) \nabla \mathbf{u}^{k-1}, \nabla \mathbf{v}_{h}\right)-\frac{1}{2}\left(\operatorname{div}\left(\mathbf{u}^{k-1}\right) \mathbf{u}^{k-1}, \mathbf{v}_{h}\right)+\left(\mathbf{f}_{0}^{n}, \mathbf{v}_{h}\right)
\end{aligned}
$$


and

$$
\begin{aligned}
( & \left.\frac{3 T_{w}}{2 \tau_{1}}, r_{h}\right)+\left(\mathbf{u}_{k-1} \nabla T_{w}, r_{h}\right)+\left(\mathbf{u}_{w} \nabla T^{k-1}, r_{h}\right)+\alpha\left(\nabla T_{w}, \nabla r_{h}\right)+\frac{1}{2}\left(\operatorname{div}\left(\mathbf{u}_{w}\right) T^{k-1}, r_{h}\right) \\
& +\frac{1}{2}\left(\operatorname{div}\left(\mathbf{u}^{k-1}\right) T_{w}, r_{h}\right) \\
= & -\left(\frac{3 T^{k-1}-4 T_{h}^{n-1}+T_{h}^{n-2}}{2 \tau}, r_{h}\right)-\left(T^{k-1} \nabla T^{k-1}, r_{h}\right)-\alpha\left(\nabla T^{k-1}, \nabla r_{h}\right) \\
& -\frac{1}{2}\left(\operatorname{div}\left(\mathbf{u}^{k-1}\right) T^{k-1}, r_{h}\right)+\left(g^{n}, r_{h}\right) .
\end{aligned}
$$

The Newton system 6.5-6.6 can be stopped at the iteration $k$ when the iterative error

$$
e r r_{w^{k}}=\frac{\left|\mathbf{u}^{k}-\mathbf{u}^{k-1}\right|_{H^{1}(\Omega)^{2}}}{\left|\mathbf{u}^{k}\right|_{H^{1}(\Omega)^{2}}}+\frac{\left|p^{k}-p^{k-1}\right|_{L^{2}(\Omega)}}{\left|p^{k}\right|_{L^{2}(\Omega)}}+\frac{\left|T^{k}-T^{k-1}\right|_{H^{1}(\Omega)}}{\left|T^{k}\right|_{H^{1}(\Omega)}}
$$

becomes smaller than a given tolerance $\varepsilon_{a}=10^{-10}\left(\operatorname{err}_{w^{k}} \leq \varepsilon_{a}\right)$.

Table 1 shows the slope of the error curve (in logarithmic scale and for $N$ going from 50 to 70 ) with a step of 5 , for different cases of $\nu(T)$ :

\begin{tabular}{|c|c|}
\hline$\nu(T)$ & $($ NSTfvd2O2) \\
\hline \hline$T+1$ & 2,4164 \\
\hline $\sin (T)+2$ & 2,1983 \\
\hline$e^{-T}+1 / 10$ & 1,9386 \\
\hline
\end{tabular}

TABLE 2. The slop of the error.

Remark 6.2. Note that the results obtained in Table 2 confirm those obtained in Theorem 5.6.

6.1.3. Comparison between order one and order two.

In the previous sections, we showed numerical validation of the first order scheme ((Eds1) and the second order scheme (5.8). In this section, we compare the effectiveness of each one.

Table 3 shows a comparison between (Eds1) with $N=200$ and (5.8) with $N=40$ and shows the corresponding CPU times of computation. We deduce that Scheme (5.8) is better then (Eds1), since (Eds2) with $N=40$ gives more precisions with much less time of computation than (Eds1).

\begin{tabular}{|c|ll|l|l|}
\hline$\nu(T)$ & scheme (Eds1), & $\mathrm{N}=200$ & scheme $(5.8)$, & $\mathrm{N}=40$ \\
\hline & error & CPU time (s) & error & CPU time (s) \\
\hline$T+1$ & 0,064 & 4106 & 0,062 & 285 \\
\hline $\sin (T)+2$ & 0,151 & 4975 & 0,051 & 274 \\
\hline$e^{-T}+1 / 10$ & 0,063 & 4192 & 0,025 & 285 \\
\hline
\end{tabular}

TABlE 3. Comparison between (Eds1) and (5.8)

6.2. Second test case. In this section, we consider the natural convection heat transfer, where the domain is a unit two-dimensional cavity of width $L$ and height $H$. The vertical walls of the cavity are isothermal, of temperatures $T_{h}$ (hot) and $T_{c}$ (cold). We refer to [11] for the details of the geometry (see Figure 7), the boundary condition and the following scaling. Using a lengthscale Lref $=H=1$ and a liquid reference state $\left(\rho_{r e f}, V_{r e f}, T_{r e f}\right)$, we can define the following scaling for the space, velocity, temperature and time variables:

$$
X=\frac{x}{L_{r e f}}, U=\frac{u}{V_{\text {ref }}}, \theta=\frac{T-T_{r e f}}{T_{h}-T_{f}}, \tau=\frac{t}{t_{\text {ref }}}, t_{\text {ref }}=\frac{L_{\text {ref }}}{V_{\text {ref }}},
$$


where $T_{\text {ref }}=\frac{T_{h}+T_{f}}{2}$ and $V_{\text {ref }}=\frac{\nu}{H}$.

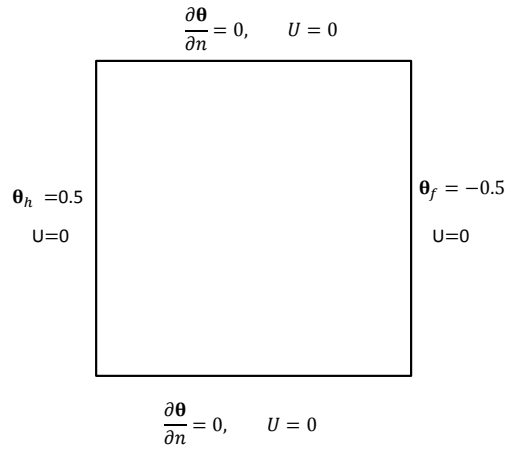

FiguRE 7. Details of the natural convection problem.

In this case, the viscosity is constant and doesn't depend on the heat but the data $\mathbf{f}$ depends of the heat as following: $\mathbf{f}_{0}=0$ and

$$
\mathbf{f}_{1}(x, \theta)=\left(\begin{array}{c}
0 \\
\frac{R a}{R e P r} \theta
\end{array}\right), \forall x \in \Omega .
$$

We take $R a=10^{6}, R e=1$ (Reynold number) and $\operatorname{Pr}=0.71$ (Prandtl number).

We first use the scheme (Eds1) to solve our problem. Figure 8 shows the repartition of the streamlines of the flow in the liquid for $N=80$. Furthermore, Figure 9 shows a comparison of the horizontal velocity profile $u(y)$ at mid-domain $x=0.5$ between our results and those obtained in [11].

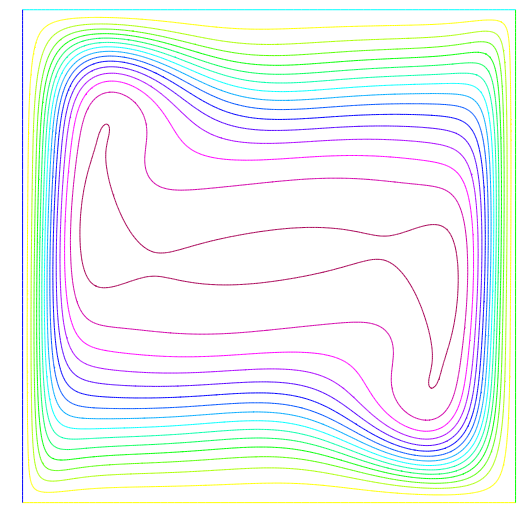

Figure 8. Streamlines of the flow in the liquid $N=80$.

Then, we solve the second-order discrete problem by using the Newton method, as follows

$$
\begin{gathered}
\left(\frac{3 \mathbf{u}_{w}}{2 \tau}, \mathbf{v}_{h}\right)+\left(\mathbf{u}_{w} \nabla \mathbf{u}^{k-1}, \mathbf{v}_{h}\right)+\left(\mathbf{u}^{k-1} \nabla \mathbf{u}_{w}, \mathbf{v}_{h}\right)+\left(\nu \nabla \mathbf{u}_{w}, \nabla \mathbf{v}_{h}\right) \\
-\left(p_{w}, \operatorname{div} \mathbf{v}_{h}\right)+\frac{1}{2}\left(\operatorname{div}\left(\mathbf{u}_{w}\right) \mathbf{u}^{k-1}, \mathbf{v}_{h}\right)+\frac{1}{2}\left(\operatorname{div}\left(\mathbf{u}^{k-1}\right) \mathbf{u}_{w}, \mathbf{v}_{h}\right)-\left(\frac{d \mathbf{f}}{d \theta}\left(\theta^{k-1}\right) \theta_{w}, \mathbf{v}_{h}\right) \\
=\left(\frac{3 \mathbf{u}^{k-1}-4 \mathbf{u}_{h}^{n-1}+\mathbf{u}_{h}^{n-2}}{2 \tau}, \mathbf{v}_{h}\right)+\left(\mathbf{u}^{k-1} \nabla \mathbf{u}^{k-1}, \mathbf{v}_{h}\right)-\left(p^{k-1}, \operatorname{div} \mathbf{v}_{h}\right) \\
\quad+\left(\nu\left(\theta_{h}^{n-1}\right) \nabla \mathbf{u}^{k-1}, \nabla \mathbf{v}_{h}\right)+\frac{1}{2}\left(\operatorname{div}\left(\mathbf{u}^{k-1}\right) \mathbf{u}^{k-1}, \mathbf{v}_{h}\right)-\left(\mathbf{f}_{1}\left(\theta_{h}^{k-1}\right), \mathbf{v}_{h}\right)
\end{gathered}
$$




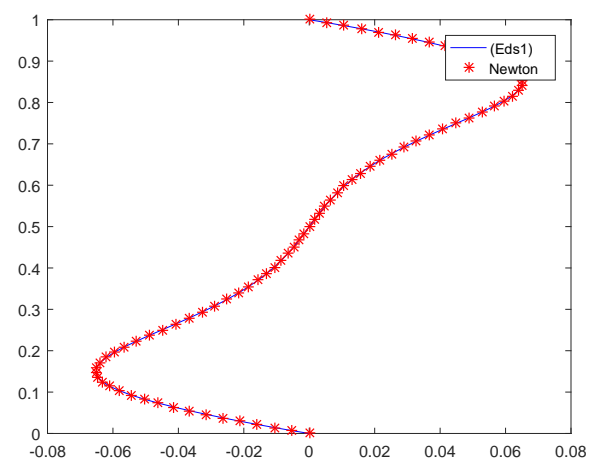

Figure 9. Comparison of the horizontal velocity profile $u(y)$ at mid-domain $x=0.5$ between (Eds1) and [11].

and

$$
\begin{aligned}
\left(\frac{3 \theta_{w}}{2 \tau}, r_{h}\right)+\left(\mathbf{u}_{k-1} \nabla \theta_{w}, r_{h}\right)+\left(\mathbf{u}_{w} \nabla \theta^{k-1}, r_{h}\right)+\alpha\left(\nabla \theta_{w}, \nabla r_{h}\right) \\
+\frac{1}{2}\left(\operatorname{div}\left(\mathbf{u}_{w}\right) \theta^{k-1}, r_{h}\right)+\frac{1}{2}\left(\operatorname{div}\left(\mathbf{u}^{k-1}\right) \theta_{w}, r_{h}\right) \\
=\left(\frac{3 \theta^{k-1}-4 \theta_{h}^{n-1}+\theta_{h}^{n-2}}{2 \tau}, r_{h}\right)+\left(\theta^{k-1} \nabla \theta^{k-1}, r_{h}\right) \\
\quad+\alpha\left(\nabla \theta^{k-1}, \nabla r_{h}\right)+\frac{1}{2}\left(\operatorname{div}\left(u^{k-1}\right) \theta^{k-1}, r_{h}\right)-\left(g^{n}, r_{h}\right)
\end{aligned}
$$

Figure 10 shows the same comparison (as in figure 9) but by using the second order scheme (5.8) and for $N=20$.

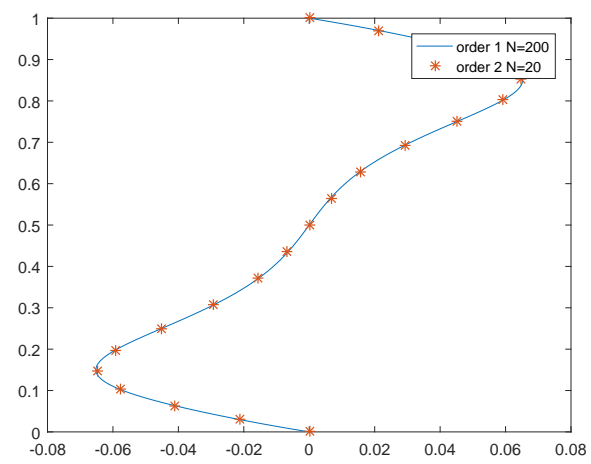

Figure 10. Comparison of the horizontal velocity profile $u(y)$ at mid-domain $x=0.5$ between the scheme (5.8) and [11].

\section{Conclusion}

In this paper we treat the fully discrete time-dependent Boussinesq problem. We introduce first order and second order schemes in space and time, and establish the corresponding a priori error estimates. Next, we perform several numerical simulations using the FreeFem ++ software to validate the theoretical results and to show that the second order scheme in much better than the first order in terms of CPU time of computation with the same precision. 


\section{REFERENCES}

[1] H. Abboud, V. Girault and T. Sayah, A second order aCCuracy in time for a full discretized time-dependent NAVier-Stockes EQuations By A two-Grid scheme. Numer. Math, 114, (2009), 189-231.

[2] R. Agroum, C. Bernardi and J. Satouri, Spectral discretization of the time-dependent Navier-Stokes problem coupled with the heat equation. Applied Mathematics and Computation, 49, (2015), 59-82.

[3] R. Agroum, S.M. Aouadi, C. Bernardi and J. Satouri, Spectral discretization of the Navier-Stokes equations coupled With the heat equation. ESAIM: Mathematical Modelling and Numerical Analysis, 49, 3, (2015), 621-639.

[4] D. Arnold, F. Brezzi \& M. Fortin, A stable finite element for the Stokes equations, Calcolo 21 (1984),337344 .

[5] C. Bernardi and V. Girault, A local regularisation operation for triangular and quadrilateral finite ELEMENTS. SIAM J. Numer. Anal., 35, (1998), 1893-1916.

[6] M. Bulicek, E. Feireisl, J. Malek, A Navier-Stokes-Fourier system for incompressible fluids with temperature dependent material coefficients, Nonlinear Analysis: Real World Applications 10, (2009), 992-1015.

[7] V. Girault \& P.-A. Raviart, Finite Element Methods for the Navier-Stokes Equations. Theory and AlgoRITHMS , in Springer Series in Computational Mathematics 5, Springer-Verlag, Berlin, (1986).

[8] V. Girault, J. L. Lions, Two-grid finite-element schemes for the steady Navier-Stokes Problem in polyheDRA. Portugaliae Mathematica, Nova Série 58,1,(2001), $25-57$.

[9] P. Clément, Approximation by finite element functions using local regularisation, R.A.I.R. O. Anal. Numer., 9, pp.77-84 (1975).

[10] F. Hecht, New development in FreeFem++, Journal of Numerical Mathematics, 20, (2012), 251-266.

[11] I. Daniala, R Molgan, F Hecht, S le Masson, A Newton method with adaptive finite elements for solving PHASE-CHANGE PROBLEMS WITH NATURAL CONVECTION. Journal of computational physics, 274 (2014) $826-840$.

[12] L.R. Scott and S. Zhang, Finite element interpolation of NOnsmooth Functions Satisfying Boundary ConDitions. Math. Comp., 54, (1990), 483-493. 